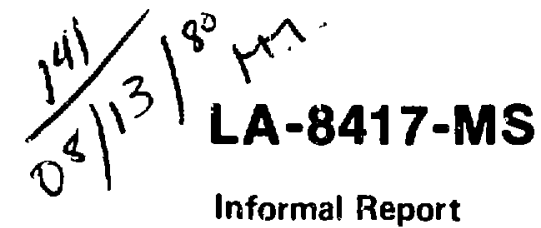

$01 \cdot 1632$

Informal Report

\title{
Gamma-Ray Spectral Calculations for Uranium Borehole Logging
}

遈

$\frac{\mathrm{A}}{\mathrm{n}}$

$\frac{2}{5}$ 
LA-8417-MS

Informal Report

UC-51

Issued: June 1980

\title{
Gamma-Ray Spectral Caleulations for Uranium Borehole Logging
}

\author{
D. A. Close \\ M. L. Evans \\ Mahavir Jain
}
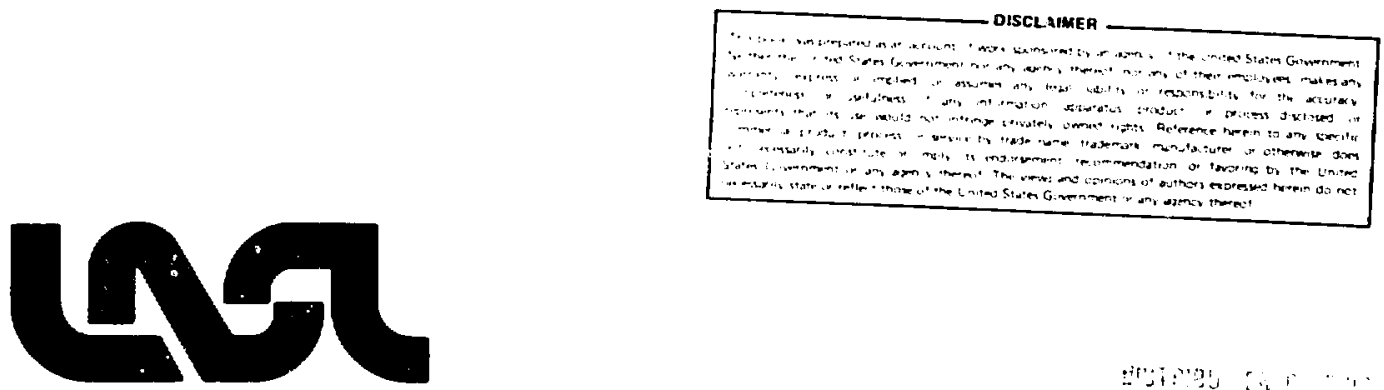


\section{CONTENTS}

ABSTRACT $\ldots \ldots \ldots \ldots \ldots \ldots \ldots \ldots \ldots \ldots \ldots \ldots \ldots \ldots \ldots \ldots \ldots \ldots \ldots \ldots \ldots$

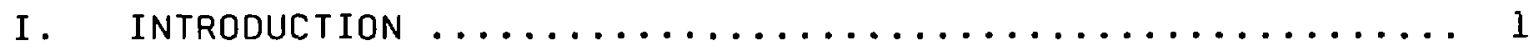

I I. BOREHOLE AND FORMATION PARAMETERS $\ldots \ldots \ldots \ldots \ldots \ldots \ldots \ldots$

II . Model Parameter studies $\ldots \ldots \ldots \ldots \ldots \ldots \ldots \ldots \ldots \ldots \ldots \ldots$

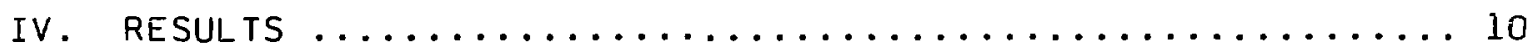

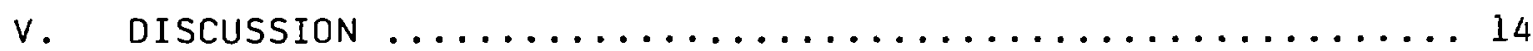

Vi. CONCLUSions AND Recommendations $\ldots \ldots \ldots \ldots \ldots \ldots \ldots \ldots \ldots$

VII. FUTURE CALCULATIONS ........................ 19

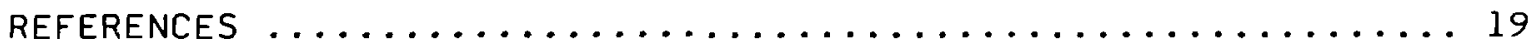

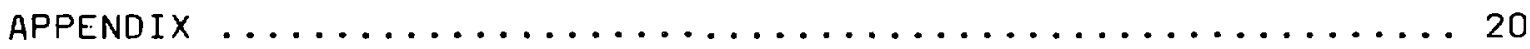

\section{TABLES}

I. Borehole and Sonde Parameters $\ldots \ldots \ldots \ldots \ldots \ldots \ldots \ldots \ldots$

II. Sandstone Elemental Concentrations $\ldots \ldots \ldots \ldots \ldots \ldots \ldots \ldots$

III. Gamma-Ray Source Spectra for Potassium,

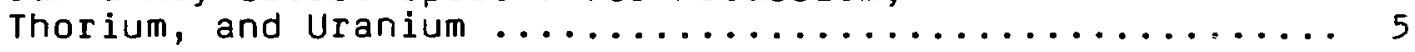

IV. Index to the Parameters of the Borehole Spectral Calculations ..................... 11 


\section{FIGURES}

1. Geometry for an infinite medium with borehole and probe... 2

2. Borehole geometry used for the ONETRAN calculations. The radius of the sonde, $R_{S}$, was held fixed at 3.334 $\mathrm{cm}$; the thickness of the sonde wall, $x_{S}$, was held fixed at $0.732 \mathrm{~cm}$; the thickness of the formation, $x_{f}$, was held fixed at $50 \mathrm{~cm}$. $R_{b}$ is the radius of the borehole

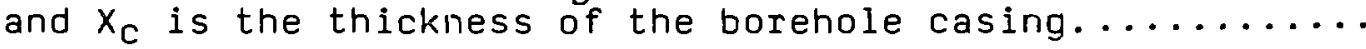

3. Energies and relative intensities of gamma rays from the

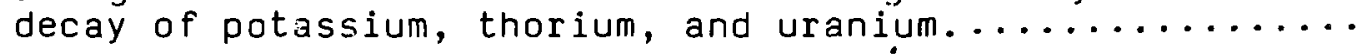

4. (a) Variation of the gamma-ray total flux with Legendre expansion order. (b) Variation of the gamma-ray total

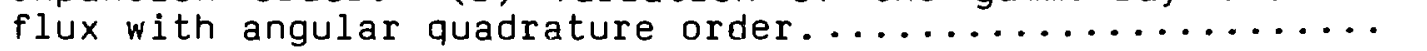

5. The ratio of the number of gamma rays crossing into the cylindrical surface of a $\mathrm{NaI}$ detector in a borehole to the number of gamma rays for no detector is plotted as a function of the gamma-ray energy. The horizontal line at 1.00 indicates the ratio expected if the NaI detector

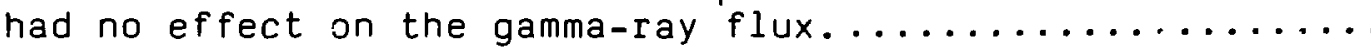

6. Gamma-ray total flux at $R=1.735 \mathrm{~cm}$ as a function of gamma-ray energy for a potassium spectrum in a sandstone formation having a porosity of 0.30 and a saturation of 1.0. The borehole parameters are borehole radius = $5.715 \mathrm{~cm}$; borehole fluid = air; borehole casing = none.... 12

7. Gamma-ray total flux at $R=1.735 \mathrm{~cm}$ as a function $n$ gamma-ray energy for a potassium spectrum in a sandstone formation having a porosity of 0.30 and a saturation of 1.0. The borehole parameters are borehole radius = $5.715 \mathrm{~cm}$; borehole fluid = water; borenole casing = none...

8. Gamma-ray total flux at $R=1.735 \mathrm{~cm}^{\circ}$ as a function of gamma-ray energy for a uranium spectrum in a sandstone formation having a porosity of 0.30 and a saturation of 1.0. The borehole parameters are borehole radius = $5.715 \mathrm{~cm}$; borehole fluid = air; borehole casing = none.... 12

9. Gamma-ray total flux at $R=1.735 \mathrm{~cm}$ as a function of gamma-ray energy for a uranium spectrum in a sandstone formation having a porosity of 0.30 and a saturation of 1.0. The borehole parameters are borehole radius = $5.715 \mathrm{~cm}$; borehole fluid = air; borehole casing $=0.318$

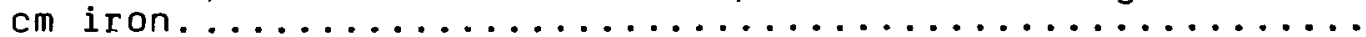


10. Gamma-ray total flux at $R=1.735 \mathrm{~cm}$ as a function of gamma-ray energy for a thorium spectrum in a sandstone formation having a porosity of 0.30 and a saturation of 1.0. The borehole parameters are borehole radius = $5.715 \mathrm{~cm}$; borehole fluid = air; borehole casing = none $\ldots 13$

11. Gamma-ray total flux at $R=1.735 \mathrm{~cm}$ as a function of gamma-ray energy for a thorium spectrum in a sandstone formation having a porosity of 0.30 and a saturation of 1.0. The borehole parameters are borehole radius = $5.715 \mathrm{~cm}$; borehole fluid = water; borehole casing =

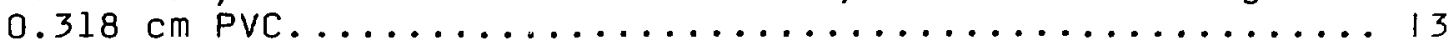

12. Variation in total gamma-ray flux at $E_{\gamma}=2.505 \mathrm{MeV}$ as a function of borehole diameter for both air-filled and water-filled boreholes........................ 15

13. Variation in total gamma-ray flux at $E_{\gamma}=1.005 \mathrm{MeV}$ as a function of borehole diameter for both air-filled and water-filled boreholes...................... 15

14. Variation in total gamma-ray flux at $E_{\gamma}=0.205 \mathrm{MeV}$ as a function of borehole diameter for both air-filled and water-filled boreholes...................... 15

15. Ratios of total flux values as a function of gamma-ray energy comparing the results of cases 20 (water-filled, no casing), 24 (air-filled, $0.318-\mathrm{cm}$ iron casing), 25 (air-filled, $0.318-\mathrm{cm}$ PVC casing), and 26 (air-filled, $0.794-\mathrm{cm}$ PVC casing) to case 15 (air-filled, no casing). The borehole diameter was held constant at $11.43 \mathrm{~cm}(4.5$ inches) and a thorium source spectrum was used......... 16

16. Ratios of total flux values as a function of gamma-ray energy comparing the results of cases 20 (water-filled, no casing) and 25 (air-filled, 0.318-cm PVC casing) to case 40 (water-filled borehole having a 0.318-cm-thick PVC casing). The borehole diameter was held constant at $11.43 \mathrm{~cm}(4.5$ inches $)$ and a thorium source spectrum was used................................ 18 
GAMMA-RAY SPECTRAL CALCULATIONS FOR URANIUM BOREHOLE LOGGING

\author{
by \\ D. A. Close, M. L. Evans, and Mahavir Jain
}

\title{
ABSTRACT
}

\begin{abstract}
Gamma-ray transport calculations were performed to determine the energy distribution of gamma rays inside a borehole introduced int.s an infinite medium. The gamma rays from the naturally occurring radioactive isotopes of potassium, thorium, and uranium were uniformly distributed in a sandstone formation (having a porosity of 0.30 and a saturation of 1.0 ) surrounding the borehole. A sonde was placed coaxially inside the borehole. Parametric studies were done to determine how the borehole radius, borehole fluid, and borehole casing influence the gamma-ray flux inside the sonde.
\end{abstract}

\section{INTRODUCTION}

Energy distributions of the gamma rays from the naturally occurring radioactive isotopes of potassium, uranium, and thorium (KUT) in infinite media of sandstone and shale have been presented. ${ }^{l}$ Subsequent calculations have determined the gamma-ray spectra when a borehole is introduced into the infinite medium, as indicated in Fig. 1. A NaI detector housed within a sonde is to be placed within the borehole. The sonde effectively removes the borehole fluid from above and below the detector.

Figure 2 shows how the actual borehole geometry of Fig. 1 has been simulated to meet the geometry requirements of the ONETRAN discrete ordinates code. ${ }^{2}$ The radii (outside dimension) of the sonde and borehole are denoted by $R_{s}$ and $R_{b}$, respectively. The thicknesses of the sonde wall, borehole casing, and formation are denoted by $x_{s}, x_{c}$, and $x_{f}$, respectively. The cylindrical 


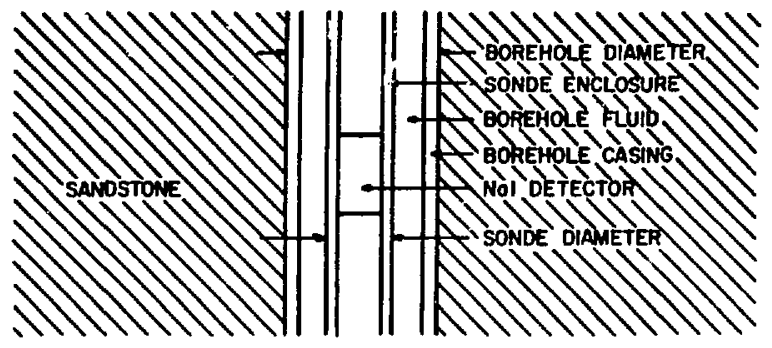

Fig. 1 .

Geometry for an infinite medium with borehole and probe.
Fig. 2 .

Borehole geometry used for the ONETRAN calculations. The radius of the sonde, $R_{S}$, was held fixed at $3.334 \mathrm{~cm}$; the thickness of the sonde wall, $x_{S}$, was held fixed at $0.732 \mathrm{~cm}$; the thickness of the formation, $X_{f}$, was held fixed at $50 \mathrm{~cm}$. $\mathrm{R}_{\mathrm{b}}$ is the radius of the borehole and $x_{C}$ is the tnickness of the borehole casing.

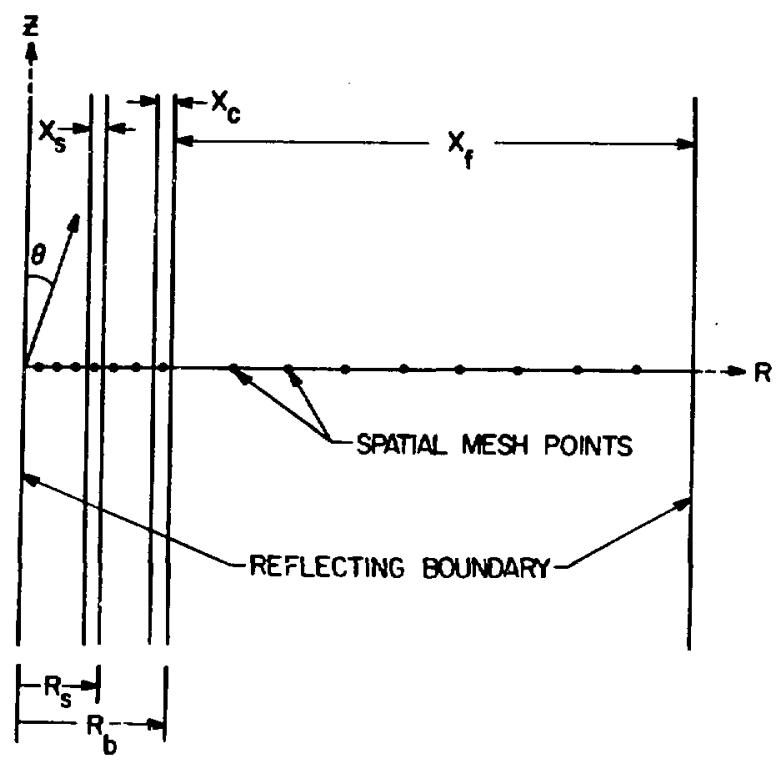

geometry option of ONETRAN has been used for these calculations. Gamma-ray fluxes are obtained as a function of the polar angle $\theta$ at each of the 15 spatial mesh points indicated in the figure.

The mechanics of performing these calculations are identical to those of the infinite-medium calculations. The many-group ONETRAN discrete ordinates code, as applied to gamma-ray transport problems and described elsewhere, 1 was used. As before, there were 265 groups, each $10 \mathrm{keV}$ wide, covering the energy range from $10 \mathrm{keV}$ to $2660 \mathrm{keV}$. 
TABLE I

BOREHOLE AND SONDE PARAMETERS

\author{
Borehole radius : $3.810,5.715,7.938,10.160,12.700 \mathrm{~cm}$ \\ Borehole fluid : air; pure water \\ Borehole casing : none; $0.318-\mathrm{cm}$ iron, $0.318-\mathrm{cm}$ \\ polyvinylchloride (PVC), $0.794-\mathrm{cm}$ \\ polyvinylchloride (PVC) \\ Sonde radius : $\quad 3.334 \mathrm{~cm}$ \\ Sonde casing : $\quad 0.732-\mathrm{cm}$ iron \\ Detector position: concentric with borehole axis
}

II . BOREHOLE AND FORMATION PARAMETERS

Table I lists the borehole parameters of interest in uranium well logging. The "standard borehole" is defined to have a radius of $5.715 \mathrm{~cm}$, no borehole fluid, and no borehole casing. The calculations were performed by holding two of these variables fixed at the above values and varying the remaining parameter. For all cases, the sonde radius and the sonde wall thickness were held fixed at the values listed in the table. Additionally, calculations were performed in which the borehole radius was varied and there was water in the borehole but no borehole casing. A NaI detector was not explicitly included in these gamma-ray flux calculations.

The elemental composition of sandstone used in these calculations is shown in Table II. This carticular sandstone formation has a porosity of 0.30 , a saturation of 1.0 , and a dry, nonporous rock density of $2.6460 \mathrm{~g} / \mathrm{cm}^{3}$. Actual sandstone includes more elements than the nine listed here, notably sodium, phosphorus, sulfur, and titanium. These four elements have been taken into. account as described below, thereby eliminating any need to make density corrections to the data. 
TABLE I I

SANDSTONE ELEMENTAL CONCENTRATIONS ${ }^{a}$

\begin{tabular}{|c|c|c|c|}
\hline Element & $\begin{array}{l}\text { Mass Density } \\
\left(\mathrm{g} / \mathrm{cm}^{3}\right) \\
\end{array}$ & $\begin{array}{l}\text { Weight Fraction } \\
(\%) \\
\end{array}$ & $\begin{array}{c}\text { Atom Fraction } \\
(\%) \\
\end{array}$ \\
\hline$H$ & 0.03357 & 1.560 & 23.502 \\
\hline c & 0.02562 & 1.190 & 1.505 \\
\hline C & 1.22543 & 56.939 & 54.047 \\
\hline $\mathrm{Mg}$ & 0.01928 & 0.896 & 0.560 \\
\hline Al & 0.04690 & 2.179 & 1.227 \\
\hline Si & 0.68240 & 31.707 & 17.145 \\
\hline K & 0.02563 & 1.191 & 0.463 \\
\hline $\mathrm{Ca}$ & 0.07500 & 3.485 & 1.321 \\
\hline $\mathrm{Fe}$ & 0.01834 & 0.852 & 0.232 \\
\hline
\end{tabular}

$a_{\text {Bulk density }}=2.1522 \mathrm{~g} / \mathrm{cm}^{3} ;$ porosity $=0.30 ;$ saturation $=$ 1.0 ; atom density $=0.08534 \times 10^{24}$ atoms $/ \mathrm{cm}^{3}$.

The four elements have been implicitly included in the composition by treating each element as though it were another element of the composition having a substantial concentration but only a slightly different $z$. For example, the sodi im $(z=11)$ concentration has been summed with that of magnesium $(z=12)$. Likewise, the density of phosphorus has been added to the density of silicon, that of sulfur added to potassium, and that of titanium to calcium. Since the concentrations of these four elements are small, including them in the sandstone in this manner changes the average $z$ of the formation slightly, from 7.6650 to 7.6886 . Over the energy range ( $10 \mathrm{keV} \leq E_{\gamma} \leq 2660 \mathrm{keV}$ ) of interest the photon 
interaction cross sections vary slowly as $z$ changes by \pm 1 . Thus, the interaction of gamma rays with this artificial composition is virtually identical to that of real sandstone. Limiting the number of elements included in sandstone reduces computation time and memory storage requirements. Also, based on results from the infinite-medium calculations, ${ }^{l}$ there was no need to treat both sandstone and shale formations and different porosities and saturations of a given formation, since the changes in the gamma-ray flux caused by these variations were small and calculable. This assertion will be verified after the gamma-ray fluxes have been enfolded with the detector response function.

\section{TABLE II I}

GAMMA-RAY SOURCE SPECTRA FOR POTASSIUM, THORIUM, AND URANIUM

Potassium

\begin{tabular}{ccc}
$\begin{array}{c}\text { Energy } \\
(\text { kev })\end{array}$ & $\begin{array}{c}\text { Relative } \\
\text { Intensity }\end{array}$ & $\begin{array}{c}\text { Parent } \\
\text { Isotope }\end{array}$ \\
\cline { 2 - 3 } 1461.0 & 0.9998 & $k 40$ \\
511.0 & 0.0002 & $k 40$
\end{tabular}

Thorium Series

\begin{tabular}{|c|c|c|}
\hline $\begin{array}{l}\text { Energy } \\
(\mathrm{keV})^{\mathrm{a}}\end{array}$ & $\begin{array}{l}\text { Relative } \\
\text { Intensity }\end{array}$ & $\begin{array}{l}\text { Parent } \\
\text { Isotope }\end{array}$ \\
\hline $\begin{array}{r}2614.5 \\
1587.9 \\
968.9 \\
964.6 \\
911.1 \\
860.5 \\
794.8 \\
727.3 \\
583.1 \\
510.7 \\
463.0 \\
338.4 \\
328.0 \\
300.1 \\
270.3 \\
241.0\end{array}$ & $\begin{array}{l}0.145 \\
0.015 \\
0.073 \\
0.023 \\
0.121 \\
0.017 \\
0.020 \\
0.027 \\
0.125 \\
0.033 \\
0.019 \\
0.050 \\
0.014 \\
0.014 \\
0.016 \\
0.015\end{array}$ & $\begin{array}{ll}\mathrm{T} l & 208 \\
\mathrm{AC} & 228 \\
\mathrm{AC} & 228 \\
\mathrm{AC} & 228 \\
\mathrm{AC} & 228 \\
\mathrm{Tl} & 208 \\
\mathrm{AC} & 228 \\
\mathrm{Bi} & 212 \\
\mathrm{~T} l & 208 \\
\mathrm{~T} & 208 \\
\mathrm{AC} & 228 \\
\mathrm{AC} & 228 \\
\mathrm{AC} & 228 \\
\mathrm{~Pb} & 212 \\
\mathrm{AC} & 228 \\
\mathrm{Ra} & 224\end{array}$ \\
\hline
\end{tabular}


Table III (continued)

Thorium Series

\begin{tabular}{ccc}
\hline $\begin{array}{l}\text { Energy } \\
\text { (kev) }\end{array}$ & $\begin{array}{l}\text { Pelative } \\
\text { Intensity }\end{array}$ & $\begin{array}{l}\text { Parent } \\
\text { Isotope }\end{array}$ \\
238.6 & 0.179 & Pb 212 \\
209.4 & 0.019 & AC 228 \\
129.1 & 0.012 & AC 228 \\
84.4 & 0.064 & Th 228
\end{tabular}

Uranium Series

\begin{tabular}{|c|c|c|}
\hline $\begin{array}{l}\text { Energy } \\
(\mathrm{keV})^{\mathrm{a}} \\
\end{array}$ & $\begin{array}{l}\text { Relative } \\
\text { Intensity }\end{array}$ & $\begin{array}{l}\text { Parent } \\
\text { Isotope }\end{array}$ \\
\hline 2448.0 & 0.008 & Bi 214 \\
\hline 2204.3 & 0.026 & $\mathrm{Bi} 214$ \\
\hline 2118.7 & 0.006 & $\mathrm{Bi} 214$ \\
\hline 1847.6 & 0.011 & $\mathrm{Bi} 214$ \\
\hline 1764.6 & 0.081 & $\mathrm{Bi} 214$ \\
\hline 1729.8 & 0.016 & $\mathrm{Bi} 214$ \\
\hline 1661.4 & 0.006 & $\mathrm{Bi} 214$ \\
\hline 1583.3 & 0.004 & $\mathrm{Bi} 214$ \\
\hline 1509.3 & 0.011 & Bi 214 \\
\hline 1408.0 & 0.013 & Bi 214 \\
\hline 1401.6 & 0.007 & Bi 214 \\
\hline $1385 . \overline{4}$ & 0.004 & Bi 214 \\
\hline 1377.7 & 0.021 & Bi. 214 \\
\hline 1281.0 & 0.007 & $\mathrm{Bi} 214$ \\
\hline 1238.2 & 0.030 & $\mathrm{Bi} 214$ \\
\hline 1155.3 & 0.008 & $\mathrm{Bi} 214$ \\
\hline 1120.4 & 0.073 & $\mathrm{Bi} 214$ \\
\hline 1001.1 & 0.004 & $\mathrm{~Pa} 234 \mathrm{~m}$ \\
\hline 934.0 & 0.015 & Bi 214 \\
\hline 806.2 & $0: 005$ & Bi 214 \\
\hline 786.0 & 0.004 & $\mathrm{~Pb} 214$ \\
\hline 768.4 & 0.023 & $\mathrm{Bi} 214$ \\
\hline 665.6 & 0.007 & $\mathrm{Bi} 214$ \\
\hline 609.4 & 0.208 & $\mathrm{Bi} 214$ \\
\hline 352.0 & 0.177 & $\mathrm{~Pb} 214$ \\
\hline 295.2 & 0.092 & $\mathrm{~Pb} \quad 214$ \\
\hline 258.8 & 0.004 & $\mathrm{~Pb} \quad 214$ \\
\hline 241.9 & 0.037 & $\mathrm{~Pb} 214$ \\
\hline $186 . \overline{0}$ & 0.019 & Ra 226 \\
\hline 185.7 & 0.012 & U 235 \\
\hline $92 . \overline{8}$ & 0.017 & Th 234 \\
\hline 92.3 & 0.015 & Th 234 \\
\hline $63 . \overline{3}$ & 0.028 & Th 234 \\
\hline
\end{tabular}

${ }^{\text {a }}$ rackets indicate gamma rays whose intensities were summed to obtain the group intensity as required by ONETRAN. 
Separate calculations were performed for the gamma-ray spectra from the naturally occurring radioactive isotopes $40_{\mathrm{K},}{ }^{232} \mathrm{Th}$, and $235,238 \mathrm{U}$.

The gamma-ray lines from these isotopes and their decay series daughters are presented in Table III and shown graphically in Fig. 3. The electron capture and $\beta^{+}$decay of $40_{K}$ results in only two gamma rays, the dominant 1461-keV gamma ray and the very weak $511-k e v$ annihilation quantum. The table lists the 20 most intense gamma rays from the thorium series and the 33 most intense gamma rays from the uranium series. These represent 95\% of the total thorium intensity and $90 \%$ of the total uranium intensity, respectively. The intensities of the lines in each spectrum have been renormaiizen to unity and are listed in the table.

\section{MODEL PARAMETER STUDIES}

Initially, calculations were performed to determine the optimum values for the $P_{n}$ and $S_{n}$ parameters of the ONETRAN code. Figure $4 a$ shows the variation of the gamma-ray total flux with $P_{n}$ (Legendre expansion order), and $F i g$. 4b shows the variation of the gamma-ray total flux with $S_{n}$ (angular quadrature order). The computer-storage requirements for the polynomial expansions of the cross sections through order ten were so large for the 265 group structure that a few-group structure (iwenty-six 


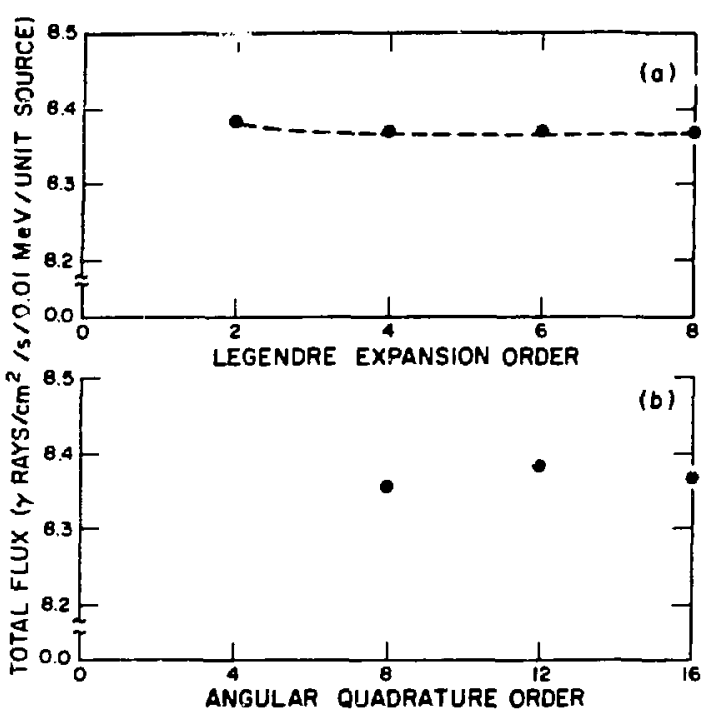

Fig. 4 .

(a) Variation of the gamma-ray total flux with Legendre exparision order. (b) variation of the gamma-ray total flux with angular quadrature order. 100-kev-wide energy bins) had to be used to determine the optimum choice of $P_{n}$. After the value of $P_{n}$ was determined, the few-group structure was also used to determine $S_{n}$. The source gamma rays had energies in the highest group, 2510-2610 kev, and their origins were uniformly distributed throughout the sandstone formation. The gamma-ray total flux has been plotted only for the highest energy group at the point $R=1.735 \mathrm{~cm}$, the mesh point just inside the sonde enclosure.

As indicated in Fig. $4 \mathrm{a}$

there is a small dependence of the gamma-ray total flux on

the Legendre expansion order. The ONETRAN solutions converge for a $\mathrm{P}_{4}$ cross-section representation. The dependence of the gamma-ray flux on the angular quadrature order, shown in Fig. 4b, indicates the flu; oscillates with quadrature order. A quadrature order of eight was arbitrarily chosen, since the true value of the flux was unknown and the variation of flux with quadrature order was smoll (less than $2 \%$ ). Alsu, an $S_{8}$ calculation requires less computer time and storage requirements than a higher quadrature order.

Fifteen spatial mesh points were chosen for the problem (see Fig. 2): three inside the sonde enclosure; one in the sonde casing; two in the region of the borehole fluid; one in the borehole casing; and eight in the sandstone formation. The placement of mesh points determines the sensitivity of the solutions to the $P_{n}$ order of the cross sections and the $S_{n}$ angular quadrature 
order. With too few spatial mesh points, the sclutions oscillate significantly, even for high $P_{n}$ and $S_{n}$ orders. With an adequate number of spatial mesh points, oscillations in tire solutions are minimal and the solutions converge for lower $P_{n}$ and $S_{n}$ orders. Furtheimore, the mesh points should be about a mean-freepath apart in a material. The 15 spatial mesh points chosen meet the mean-free-path criterion and, combined with a $\mathrm{P}_{4} \mathrm{~S}_{8}$ calculation, minimize the oscillations in the solutions.

The preceding discussion assumed that a NaI detector placed inside the sonde would have a negligible effect on the gamma-ray flux inside the sonde. Two Monte carlo ${ }^{3}$ calculations were performed to determine the validity of this assumption. The incident gamma-ray energy for these studies was $609 \mathrm{keV}$. This is the most intense gamma ray in the uranium series and is more intense than any line in the thorium series. Furthermore, it is relatively low in energy and should be more sensitive to the presence of a NaI detector than a higher-energy gamma ray. The detector was assumed to have a diameter of $5.08 \mathrm{~cm}$ and a length of $25.40 \mathrm{~cm}$, which is the largest jetector currently envisioned for use downhole. The number of gamma rays crossing into the cylindrical surface of the detector was tallied in thirteen 50-kev-wide energy bins. These studies assumed a 3.334-cm-radius sonde with a $0.732-$ cm-tinick iron wall; the borehole radius was $5.715 \mathrm{~cm}$ and there was no borehole casing or borehoie fluid.

The results of this study are shown in Fig. 5. The ratio of the number of gamma rays crossing into the cylindrical surface of the NaI detector to the number of gamma rays for no detector in the borehole is plotted as a function of the gamma-ray energy. If the detector had no effect on ihe gamma-ray flux, this ratio would be unity for all energies. This limiting value is indicated by the horizontal line.

The errors shown in Fig. 5 were calculated assuming uncorrelated event samples for the two Monte Carlo calculations. However, the two event samples were strongly correlated, especially at high energies. Thus, the errors shown in Fig. 5 are gross 


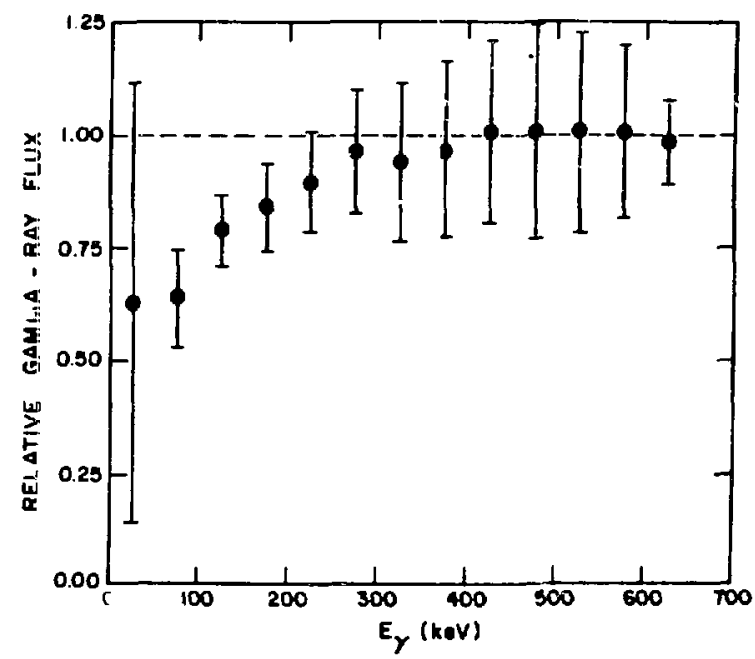

Fig. 5 .

The ratio of the number of gamma rays crossing into the cylindrical surface of a NaI detector in a bo ehole to the number of gamme rays for no detector is plotted as a function of the gamma-ray energy. The horizontal line at i.00 indicates the ratio expected if the NaI detector had no effect on the gamma-ray flux.

overestimates of the true uncertainty in the relative gamma-ray flux.

There is a negligible effect on gamma rays having energies greater than $200 \mathrm{keV}$. The presence of the NaI detector reduces the gamma-ray flux for gamma rays having energies less than 200 kev. These results are indicative of the maximum effect a detector can have on the flux, since most detectors placed downhole: will be significantly smaller in size, for example, $2.54 \mathrm{~cm}$ $\times 12.70 \mathrm{~cm}$. From these studies, it is felt that the effect of a NaI detector on the steady-state flux in a borehole can be ignored.

IV. RESULTS

A total of 40 borehole gammi-ray spectral calculations was performed for sandstone. Table IV lists the parameters of each of the case studies, grouped by spectral source type (except for case 40, which was performed subsequent to the campletion of the initial series of 13 case studies for each spectral source). Shown in Figs. 6-1l are the gamma-ray total flux spectra at a point just inside the sonde wall ( $R=1.735 \mathrm{~cm})$ for a representative sample of the 40 cases of Table IV. The sample demonstrates the changes in flux caused by a relatively large change in 
TABLE IV

INIJEX TO THE PARAMETERS OF THE

BOREHOLE SPECTRAL CALCULATIONS

\begin{tabular}{|c|c|c|c|c|c|c|}
\hline \multirow[b]{2}{*}{ Case \# } & \multirow{2}{*}{$\begin{array}{c}\text { Gamma-Ray } \\
\text { Source }\end{array}$} & \multicolumn{5}{|c|}{ Borehole } \\
\hline & & Radius (cm) & Fluid & Cas & sing & \\
\hline 1 & K & 3.810 & Air & None & & \\
\hline 2 & K & 5.715 & Air & None & & \\
\hline 3 & K & 7.938 & Air & None & & \\
\hline 4 & K & 10.150 & Air & None & & \\
\hline 5 & K & 12.700 & Air & None & & \\
\hline 6 & $\mathrm{~K}$ & 3.810 & Water. & None & & \\
\hline 7 & $\mathrm{~K}$ & 5.715 & Water & None & & \\
\hline 8 & $k$ & 7.938 & Water & None & & \\
\hline 9 & $k$ & 10.160 & Water & None & & \\
\hline 10 & $k$ & 12.700 & water & None & & \\
\hline 11 & $k$ & 5.715 & Ai工 & 0.318 & $c n_{1}$ & Iror \\
\hline 12 & $k$ & 5.715 & Air & 0.318 & $\mathrm{Cm}$ & PVC \\
\hline 13 & $k$ & 5.715 & Air & 0.794 & $\mathrm{~cm}$ & $P V C$ \\
\hline 14 & Th & 3.810 & Air & None & & C \\
\hline 15 & Th & 5.71 .5 & Ais & None & & \\
\hline 16 & Th & 7.938 & AiI & None & & \\
\hline 17 & Th & 10.160 & Air & None & & \\
\hline 18 & Th & 12.700 & Air & None & & \\
\hline 19 & Th & 3.810 & Water & Norie & & \\
\hline 20 & Th & 5.715 & Water & None & & \\
\hline 21 & Th & 7.938 & Water & None & & \\
\hline 22 & Th & 10.160 & water & None & & \\
\hline 23 & Th & 12.700 & Water & None & & \\
\hline 24 & Th & 5.715 & Air & 0.318 & $\mathrm{~cm}$ & Iron \\
\hline 25 & Th & 5.715 & Air & 0.318 & $\mathrm{~cm}$ & PVC \\
\hline 26 & $T r$ & 5.715 & Air & 0.794 & $\mathrm{~cm}$ & PVC \\
\hline 27 & $U$ & 3.810 & Air & None & & \\
\hline 28 & U & 5.715 & Air & None & & \\
\hline 29 & $U$ & 7.938 & Air & None & & \\
\hline 30 & U & 10.160 & Air & None & & \\
\hline 31 & $U$ & 12.700 & Air & None & & \\
\hline 32 & $U$ & 3.810 & Water & None & & \\
\hline 33 & $U$ & 5.715 & Water & None & & \\
\hline 34 & $U$ & 7.938 & Water & None & & \\
\hline 35 & $U$ & 10.160 & Water & None & & \\
\hline 36 & $U$ & 12.700 & Water & None & & \\
\hline 37 & $U$ & 5.715 & Air & 0.318 & $\mathrm{~cm}$ & Iron \\
\hline 38 & U & 5.715 & Air & 0.318 & $\mathrm{~cm}$ & PVC \\
\hline 39 & $\mathrm{U}$ & 5.715 & Air & 0.794 & $\mathrm{~cm}$ & PVC \\
\hline 40 & Th & 5.715 & Water & 0.318 & $\mathrm{~cm}$ & PVC \\
\hline
\end{tabular}




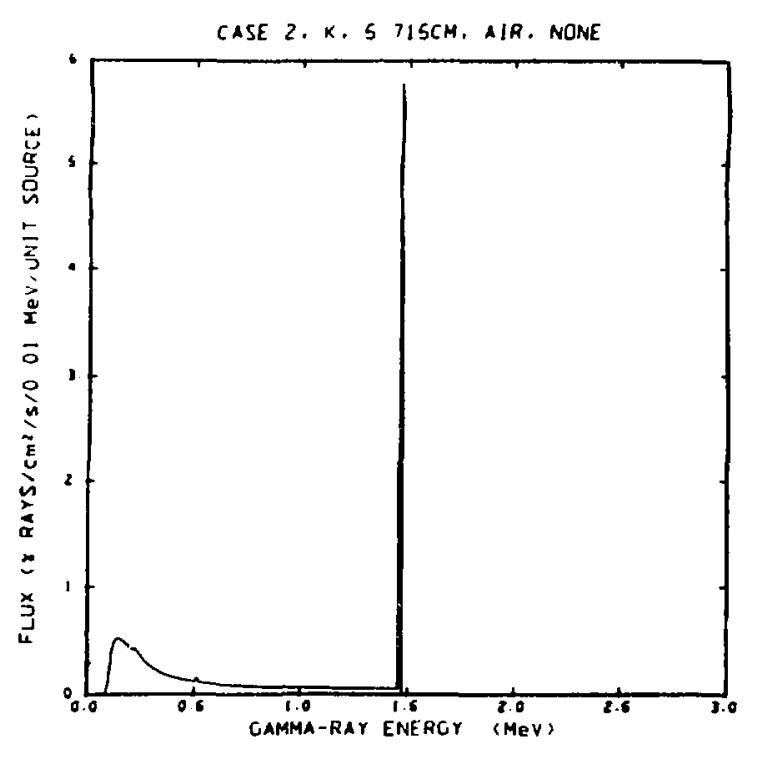

Fig. 6.

Gamma-ray total flux at $R=$ $1.735 \mathrm{~cm}$ as a function of gamma-ray energy for a potassium spectrum in a sandstone formation having a porosity of 0.30 and a saturation of 1.0 . The borehole parameters are borehole radius $=5.715 \mathrm{~cm}$; borehole fluid = air; borehole casing = none.

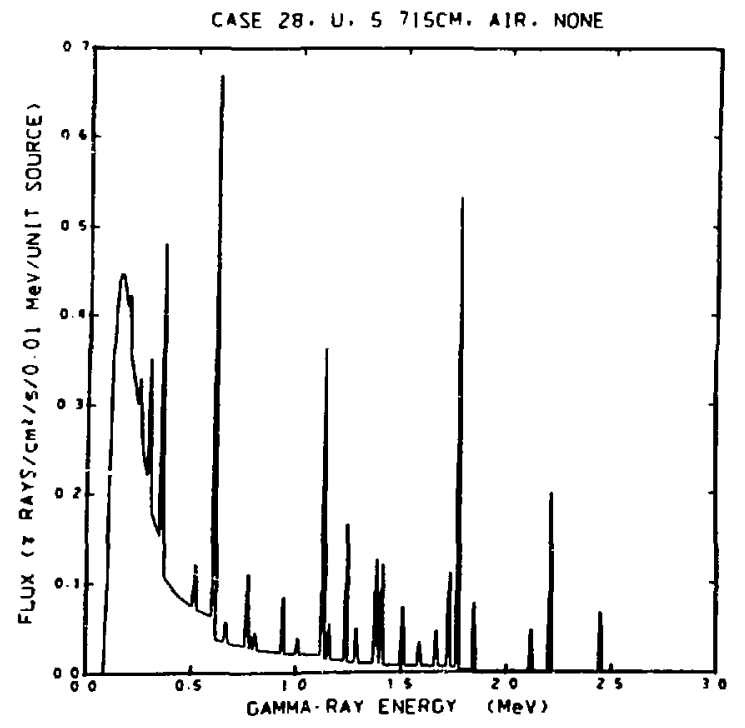

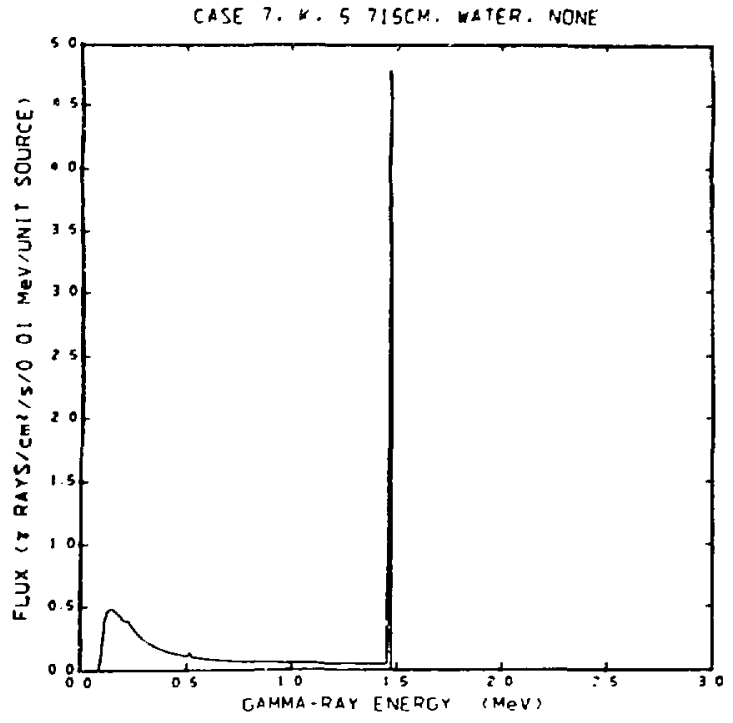

Fig. 7 .

Gamma-ray total flux at $R=$ $1.735 \mathrm{~cm}$ as a function of gamma-ray energy for a potassium spectrum in a sandstone formation having a porosity of 0.30 and a saturation of 1.0 . The borehole parameters are: borehole radius $=5.715 \mathrm{~cm}$; borehole fluid = water; borehole casing = none.

Fig. 8 .

Gamma-ray total flux at $R=$ $1.735 \mathrm{~cm}$ as a function of gamma-ray energy for a uranium spectrum in a sandstone formation having a porosity of 0.30 and a saturation of 1.0. The borehole parameters are borehole radius $=5.715 \mathrm{~cm}$; borehole fluid = air; borehole casing = none. 


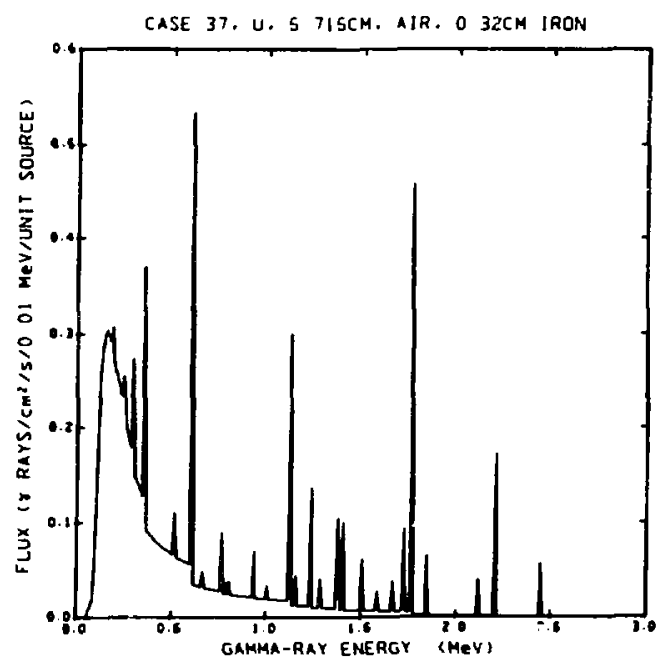

Fig. 9.

Gamma-ray total flux at $R=$ $1.735 \mathrm{~cm}$ as a function of gamma-ray energy for a uranium spectrum in a sandstone formation having a porosity of 0.30 and a saturation of 1.0. The boretole parameters are borehole radius $=5.715 \mathrm{~cm}$; borehole fluid = air; borehole casing $=0.318 \mathrm{~cm}$ iron.

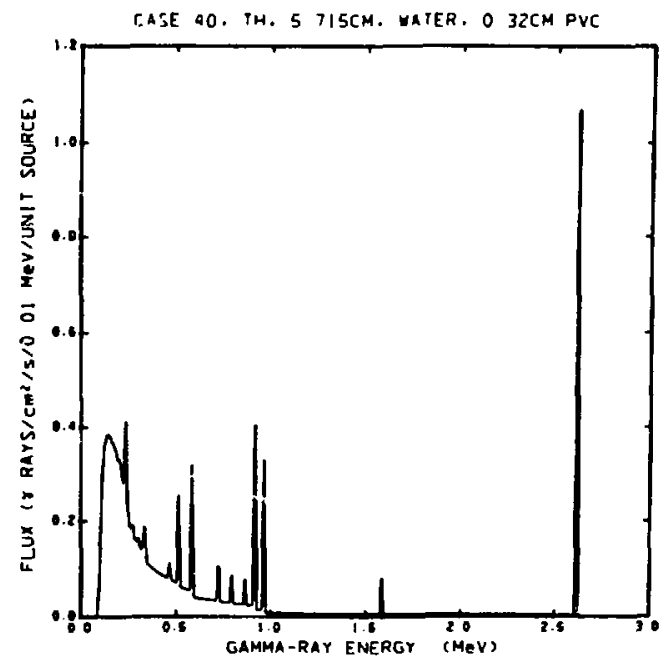

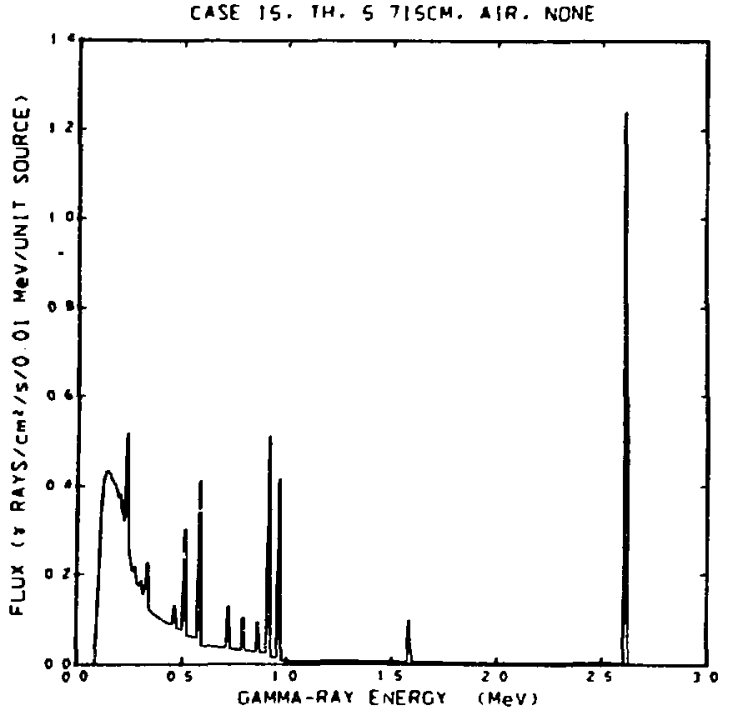

Fig. 10.

Gamma-ray total flux at $R=$ $1.735 \mathrm{~cm}$ as a function of gamma-ray energy for a thorium spectrum in a sandstone formation having a porosity of 0.30 and a saturation of 1.0. The borehole parameters are borehole radius $=5.715 \mathrm{~cm}$; borehole fluid = air; borehole casing = none.

Fig. 11 .

Gamma-ray total flux at $R=$ $1.735 \mathrm{~cm}$ as a function of gamma-ray energy for a thorium spectrum in a sandstone formatic. having a porosity of 0.30 and a saturation of 1.0. The borehole parameters are borenole radius $=5.715 \mathrm{~cm}$; borehole fluid = water; borehole casing $=0.318 \mathrm{~cm}$ PVC. 
borehole parameters. All calculations assumed a "UNIT SOURCE," defined to be one gamma ray per second per $\mathrm{cm}^{3}$ of the formation. The spectral printouts of the total flux at a point just inside the sonde wall ( $R=1.735 \mathrm{~cm}$ ) are given in the Appendix for all 40 case studies. These data allow quantitative comparisons to be made that demonstrate the effects that borehole radius, borehole fluid, and borehole casing have on the gamma-ray flux inside the sonde.

\section{v. DISCUSSION}

Interpretation of spectral gamma-ray logs relies on energydependent correction factors relating the spectrum obtained in an unknown borehole to that from a calibration test pit. The results of this study provide spectral correction data for changes in borehole parameters (i.e., boreholo diameter, fluid, and casing). Previous calculations ${ }^{l}$ have determined these correction factors for changes in $\bar{Z}$ and density of the formation caused by variations in formation porosity, fluid saturation, and elemental composition.

Correcting gamma-ray logs for effects caused by varying borehole diameter is an important aspect of gamma-ray logging analysis and interpretation. Figures 12-14 demonstrate these effects at three gamma-ray energies for both air-and water-filled borehoies. A thorium source spectrum was used to obtain the results shown in Figs. 12-14, since that spectrum affords the broadest range of gamma-ray energies compared with the potassium and uranium spectra. The results were obtained with an uncased borehole.

At 2.505 MeV, the total gamma-ray flux decreases very slightly ( $20.5 \%)$ for air-filled boreholes over the range of borehole diameters studied (i.e., $7.62-25.40 \mathrm{~cm}$ ). The flux increases about $0.8 \%$ at $1.005 \mathrm{MeV}$ and about $3.2 \%$ at $0.205 \mathrm{MeV}$ for the same range of borehole diameters. These small variations in total flux are due to slight perturbations in the effective path length of the gamma rays for the different borehole diameters.

The total flux decreases almost linearly with increasing borehole diameter for water-filled boreholes. At the higher 


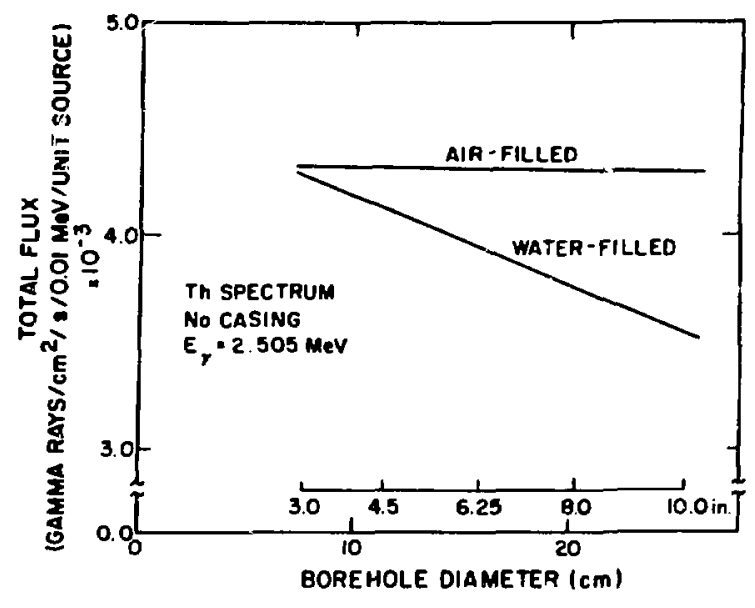

Fig. 12 .

variation in total gamma-ray flux at $E_{\gamma}=2.505$ MeV as a function of borehole diameter for both air-filled and waterfilled boreholes.

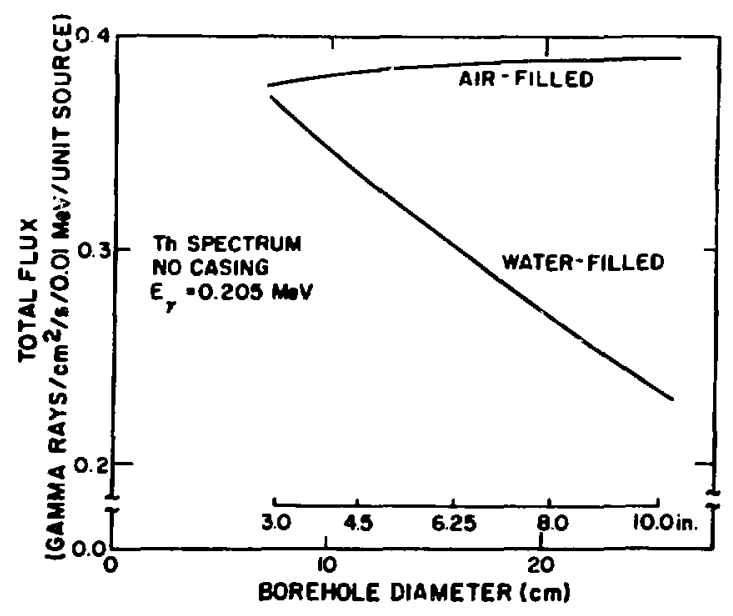

Fig. 14 .

variation in total gamma-ray flux at $E_{\gamma}=0.205$ MeV as a function of borehole diameter for both air.filled and water.. filled boreholes.

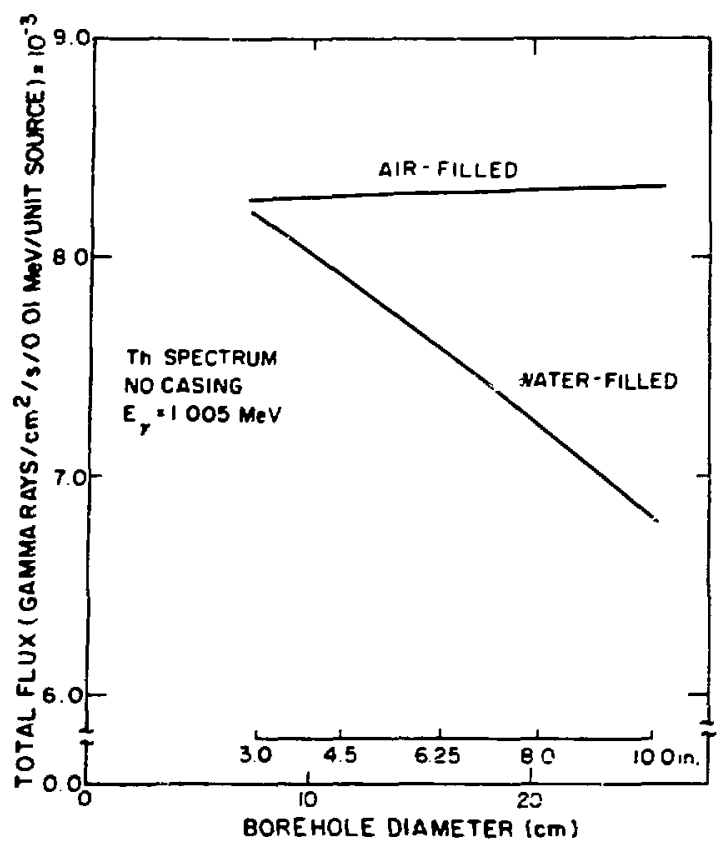

Fig. 13.

variation in total gamma-ray flux at $E_{\gamma}=1.005 \mathrm{MeV}$ as a function of borehole diameter for both air-filled and waterfilled boreholes.

energies the attenuation of the gamma-ray flux increases by about $20 \%$ as the borehole diameter increases from 7.62 $\mathrm{cm}$ to $2.5 .40 \mathrm{~cm}$. The attenuation is more pronounced at $0.205 \mathrm{MeV}$, as expected, resulting in a drop in total flux of about $40 \%$ over the range of borehole diameters studied.

The gamma-ray total flux for an air-filled, uncased borehole (case 15) is compared 


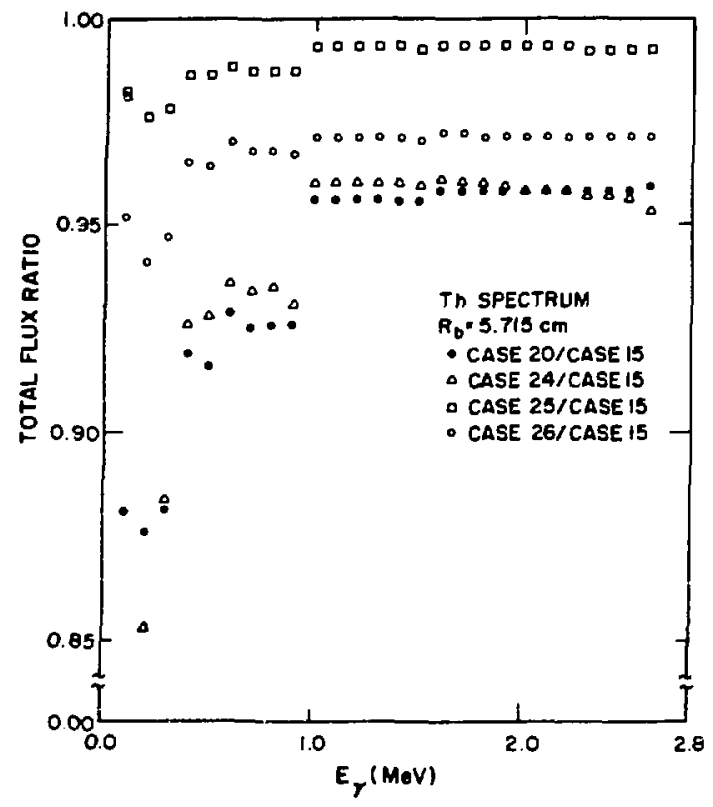

Fig. 15 .

Ratios of total flux values as a function of gamma-ray energy comparing the results of cases 20 (water-filled, no casing), 24 (air-filled, $0.318-\mathrm{cm}$ iron casing), 25 (air-filled, 0.318cm PVC casing), and 26 (airfilled, $0.794-\mathrm{cm}$ PVC casing) to case 15 (air-filled, no casing). The borehole diameter was held constant at $11.43 \mathrm{~cm}$ ( 4.5 inches) and a thorium source spectrum was used. in Fig. 15 with that for a water-filled, uncased borehole and with those for air-filled boreholes having different casings. The figure shows the total flux ratio between case 15 and each of the cases 20 (water-filled, no casing), 24 (air-filled, $\quad 0.318-\mathrm{cm}$ iron casing), 25 (air-filled, 0.318$\mathrm{cm}$ PVC casing), and 26 (airfilled, $0.794-\mathrm{cm}$ PVC casing) as a function of gamma-ray energy. A thorium source spectrum and a constant borehole diameter of $11.43 \mathrm{~cm} \quad(4.5$ inches) was used for each case.

The water-filled, uncased borehole (case 20) is the most attenuating geometry, even more attenuating than the $0.318-\mathrm{cm}$ ( $1 / 8$ inch)-thick iron casing of case 24. The 0.318-cm-thick polyvinylchloride casing with an air-filled borehole (case 25) is the least attenuating geometry.

The flux ratio (Fig. 15) is highly energy dependent for all comparisons. The general shape of each ratio curve is that resulting from increasing photoelectric absorption at low energies and increasing pair-production absorption at high energies (discernible only for case 24). (See Ref. 1 for a more detailed explanation.) Detailed structure is evident in all curves but is especially obvious for the more highly attenuating geometries. The apparent discontinuities in the ratios are associated with the occurrence of discrete lines in the thorium source spectrum 
(see Fig. 3). Since the energy region just above 1 MeV in the flux spectrum of case 15 contains only downscattered radiation, while the regior: just below $1 M=V$ contains contributions from both downscattering and the presence of source lines in the region, the two energy regions contain grossly different quantities of flux. As the borehole attenuation increases relative to that of case 15 , the additional quantity of flux downscattered into the two regions is very nearly the same owing to the slowly varying energy dependence of the compton cross section. Therefare, the fractional increase in flux in the two regions is different. On the other hand, the fractional amount of flux removed from the two energy regions by photoelectric absorption and compton downscattering is roughly the same. The net imbalance in flux caused by these two competing processes results in the discontinuities observed between cases differing in borehole attenuation. Thus, it is clear that simple exponential attenuation calculations that estimate only the amount of flux absorbed and completely ignores the complex initial spectrum and the downscattering of higher energy gamma rays and the subsequent buildup factor cannot adequately describe the changes in spectral shape resulting from varying borehole attenuation. A transport calculation that includes the effects of downscatter and the complexity of the source spectrum is required to obtain accurate reults.

The total flux ratio between case 40 (water-filled, 0.318-cmthick polyvinylchloride casing) and case 20 (water-filled, uncased) is shown in Fig. 16. Also shown in this figure is the total flux ratio between case 40 (water-filled, 0.318-cm-thick polyvinylchloride casing) and case 25 (air-filled, 0.3l8-cm-thick polyvinylchloride casing). Again, the borehole diameter was held constant at $11.43 \mathrm{~cm}$ and a thorium source spectrum was used for the calculations.

The features of these two curves are very similar to those observed earlier in Fig. 15. The inclusion of a 0.318-cm-thick polyvinylchioride casing in case 40 produces little additional attenuation over that observed in case 20 for a water-filled borehole with no casing. On the other hand, a water-filled borehole 


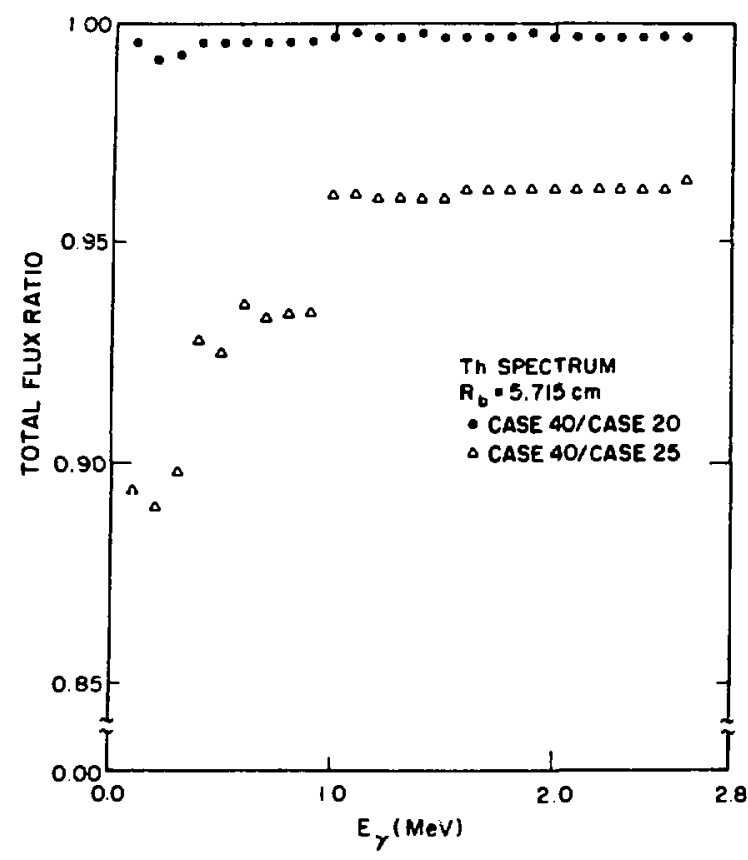

Fig. 16 .

Ratios of total flux values as a function of gamma-ray energy comparing the results of cases 20 (water-filled, no casing) and 25 (air-filled, $0.318-\mathrm{cm}$ PVC casing) to case 40 (waterfilled borehole having a 0.318cm-thick PVC casing). The borehole diameter was held constant at $11.43 \mathrm{~cm} \quad(4.5$ inches) and a thorium source spectrum was used. with

a

$0.318-c m-t h i c k$

polyvinylchloride casing is significantly more attenuating than an air-filled borehole with the same casing. This was observed before for a water-filled, uncased borehole versus an air-filled, uricased borehole.

VI. CONCLUSIONS AND

\section{RECOMMENDATIONS}

Parameteric studies performed to determine the effects of borehole diameter, fluid, and casing on KUT flux spectra observed in a logging sonde lead to the following conclusions:

A.) Corrections to the flux spectra observed in airfilled, uncased boreholes having diameters in the range $7.62-25.40 \mathrm{~cm}(3.0$ 10.0 inches) are unnecessary above $500 \mathrm{keV}$ if uncertainties in the flux values up to $1 \%$ can be tolerated.

B.) Corrections to the flux spectra observed in water-filled, uncased boreholes having diameters in the range 7.62-25.40 $\mathrm{cm}$ (3.0-10.0 inches) vary linearly with borehole diameter to within $2 \%$ for energies greater than $100 \mathrm{keV}$.

C.) In an 1l.43-cm-diameter (4.5 inch), air-filled, uncased borehole, corrections to the flux spectra for water filling are 
larger than those for casing the borehole with up to $0.318-\mathrm{cm}$ ( $1 / 8$ inch) iron.

D.) Corrections to flux spectra for differences in borehole geometry and compositions are dependent on the distribution of spectral lines in the source. Simple exponential attenuation cannot account for the changes in flux. Hence, transport calculations are necessary to obtain reasonable estimates of spectral flux changes resulting from variations in borehole diameter, borehole fluid, and borehole casing.

\section{FUTURE CAL.CULATIONS}

The gamma-ray fluxes presented in this report are insufficient to be used directly to aid in the interpretation of borehole logging data. They must first be convoluted with a gamma-ray detector response function to yield a calculated spectrum comoarable to that measured by a $\mathrm{NaI}$ detector placed downhole. In addition to che magnitude of the gamma-ray fluxes, such a calculation needs as input the angular distribution of the gamma rays incident on the detector. These angular fluxes have been written into data files for the cases described above. The files have been saved on magnetic disk and tape in a format described earlier. ${ }^{1}$ A subsequent report will present the results of the convolution of these gamma-ray fluxes with a gamma-ray detector response function.

\section{REFERENCES}

1. M. Jain, M. L. Evans, and D. A. Close, "Nondestructive Assay Technology for Uranium Resource Evaluation, Infinite Medium Calculations, Final Report," Los Alamos Scientific Laboratory report LA-7713-MS (March 1979).

2. T. R. Hill, "ONETRAN: A Discrete ordinates Finite Element Code for the Solution of the One-Dimensional Multigroup Transport Equation," Los Alamos Scientific Laboratory report LA-5990-MS (June 1975). 
3. E. D. Cashwell, J. R. Neergaard, W. M. Taylor, and G. D. Turner, "MCN: A Neutron Monte! Carlo Code," Los Alamos Scientific Laboratory report LA-475I (January 1972); E. D. Cashwell, J. R. Neergaard, C. J. Everett, R. G. Schrandt, W. M. Taylor, and G. D. Turner, "Monte Carlo Photon Codes: MCG and MCP," LoS Alamos Scientific Laboratory report LA-5157-MS (March 1973).

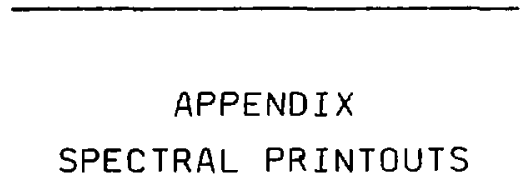

Included below are computer printouts of the total flux values for each spectrum of the 40 borehole calculations. The data for each spectrum are printed on a single page with descriptive information including case number, source spectrum ( $K$, $U$, or Th), borehole radius, borehole" fluid, and borehole casing. The values of energy listed are the centers of the lo-kev-wide bins used in the ONETRAN calculations. The units for the printouts are identical to those of the spectral plots of Figs. 6-11, that is, MeV for the energy values and gamma rays/cm $\mathrm{cm}^{2} / \mathrm{s} / 0.01 \mathrm{MeV} / \mathrm{unit}$ source for the flux values. 


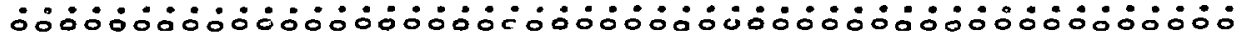

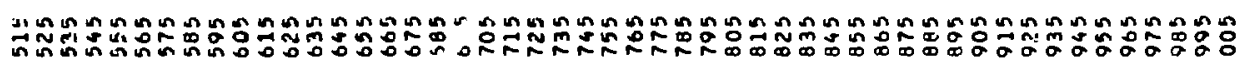

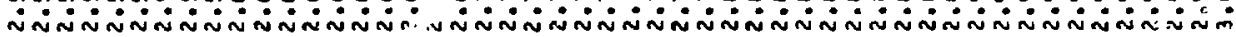

$\stackrel{\times}{\overrightarrow{4}}$

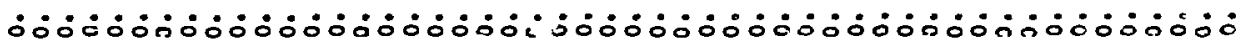

它

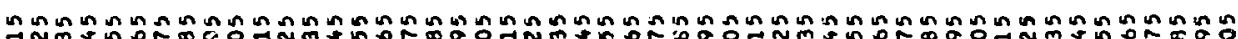

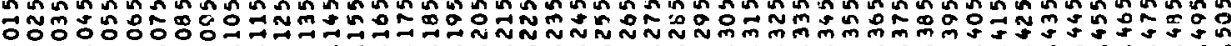

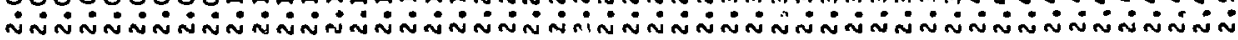

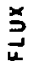

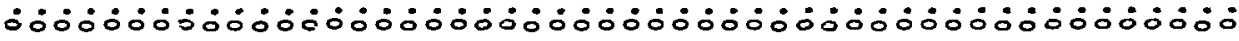

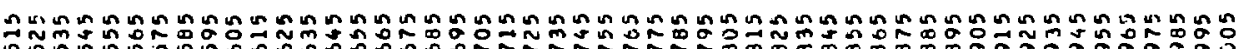

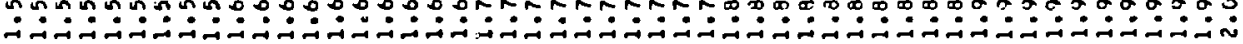

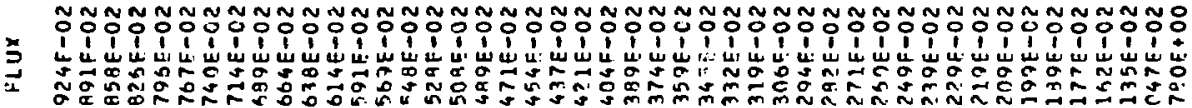

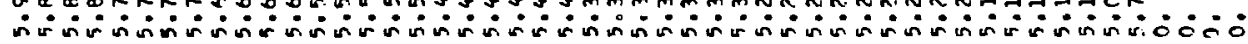

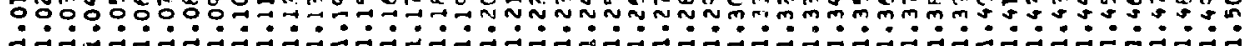

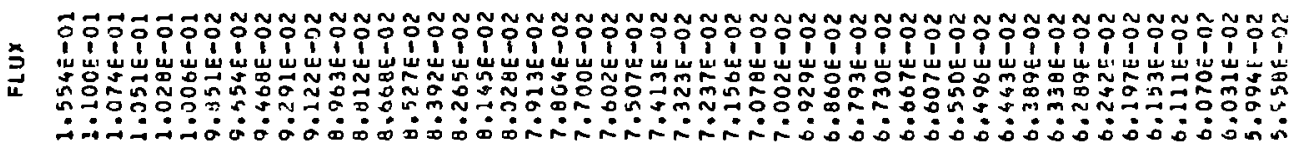

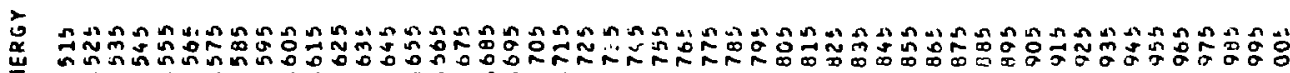

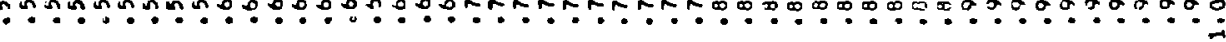

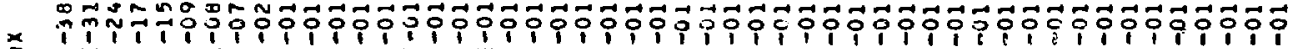

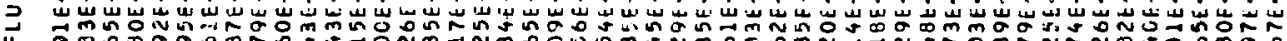

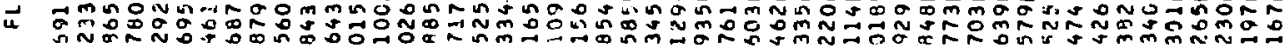

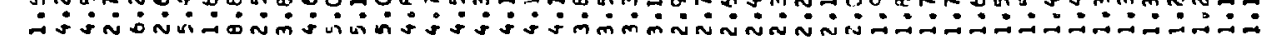




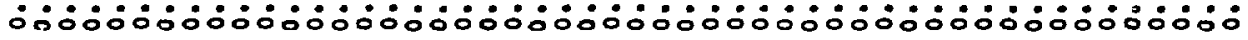

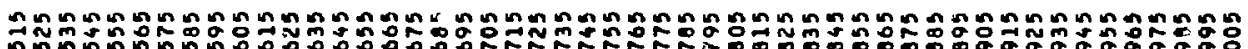

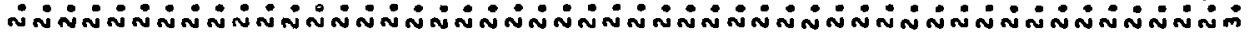

$\stackrel{\times}{3}$

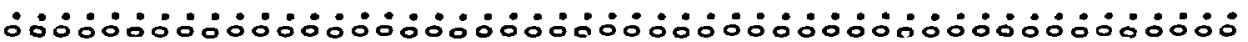

勇

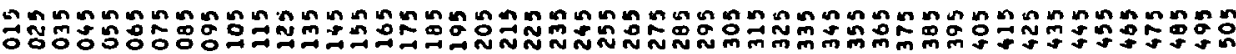

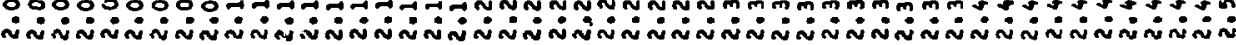

$\stackrel{\times}{\vec{z}}$

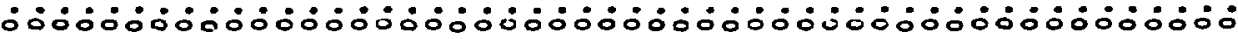

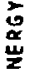

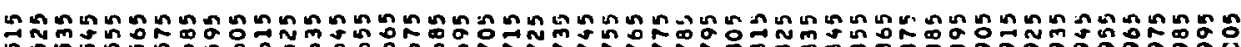

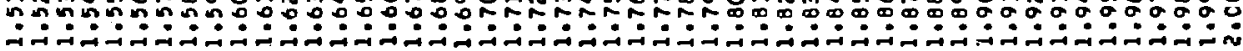

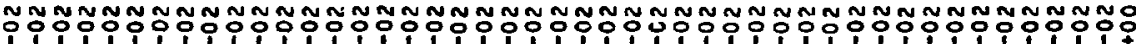

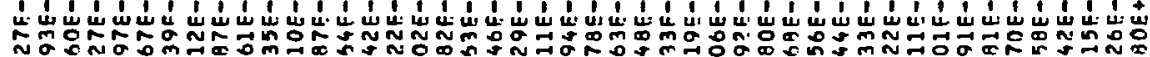

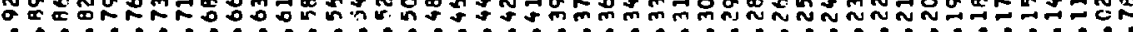

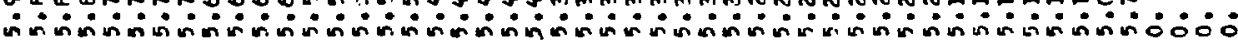

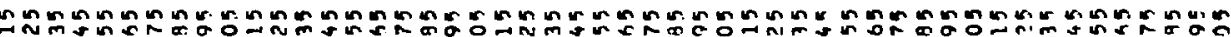

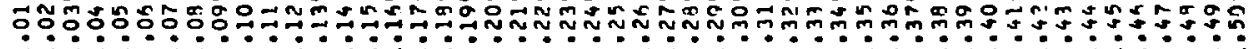

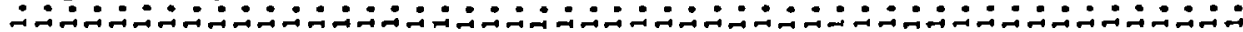

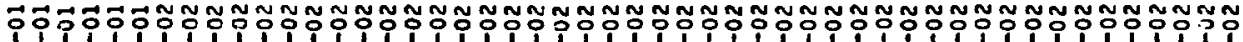

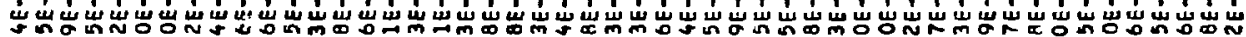

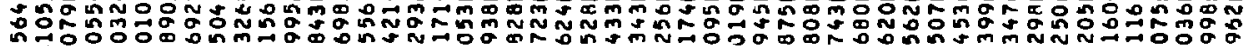

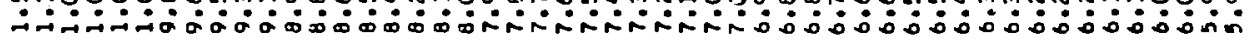

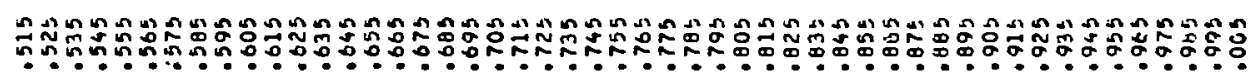
(a)

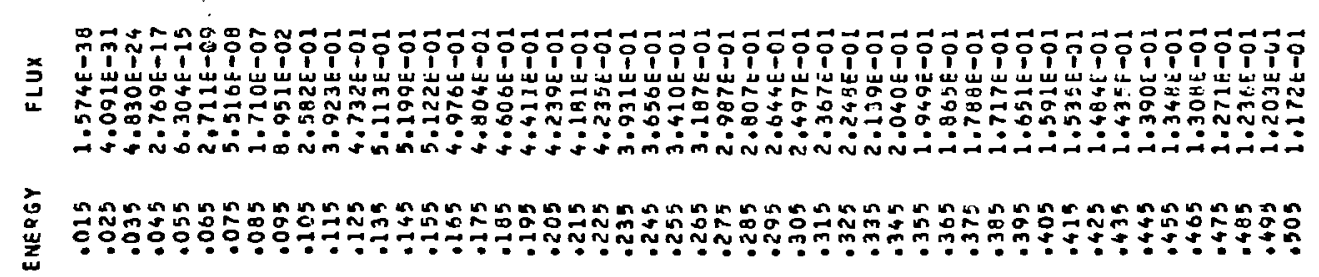




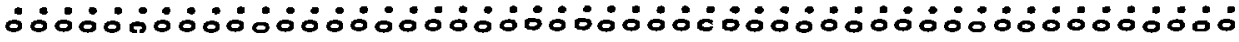

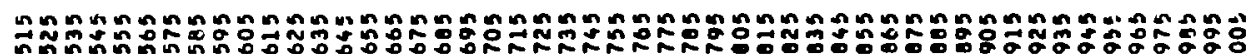

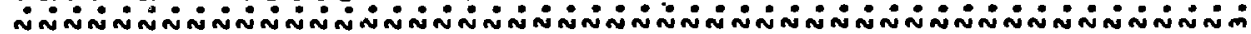

$\stackrel{3}{3}$

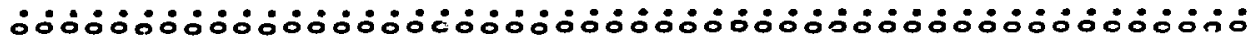

总

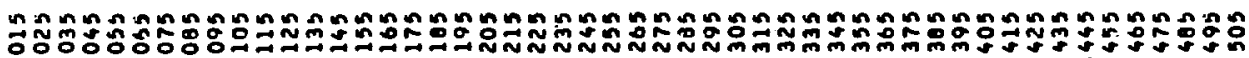

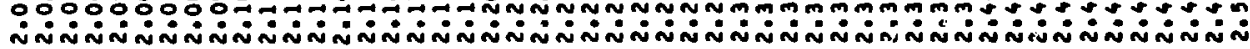

$\stackrel{\times}{3}$

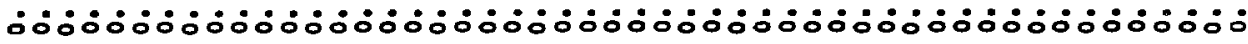
క̀

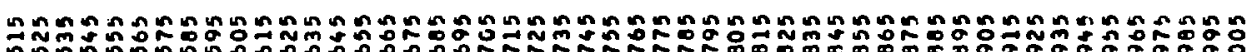

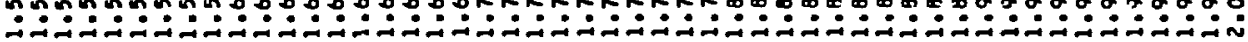

N

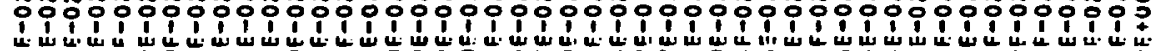

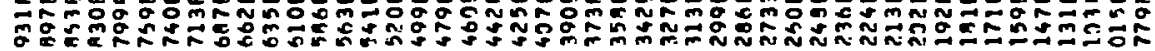

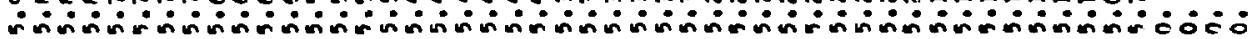

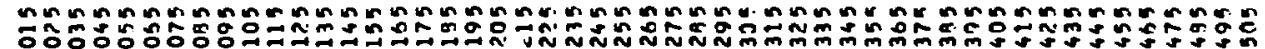

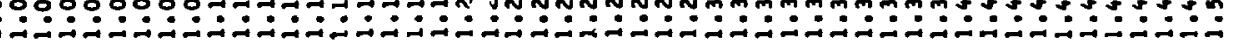

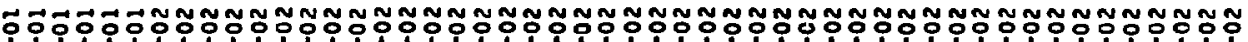

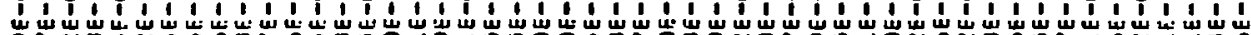

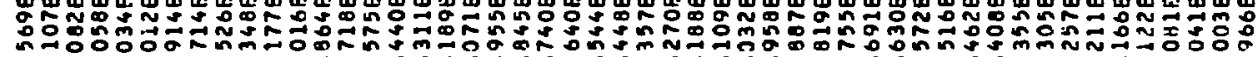

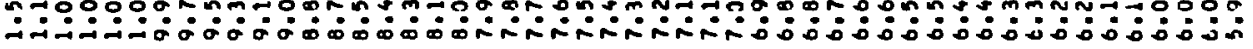

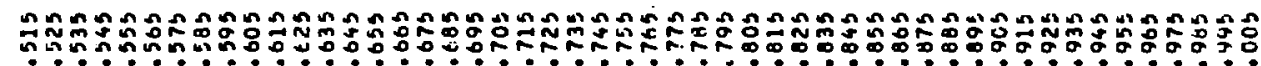

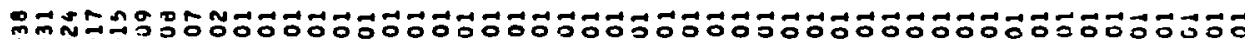

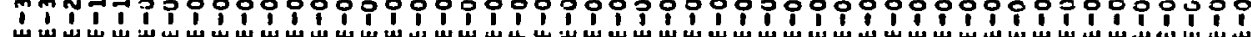

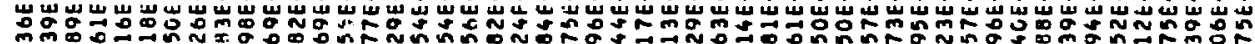

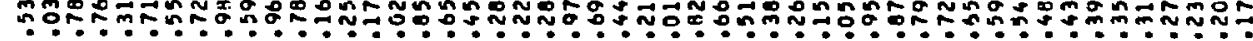

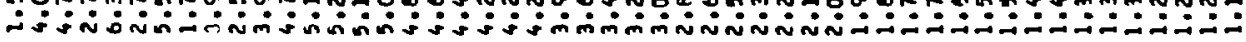

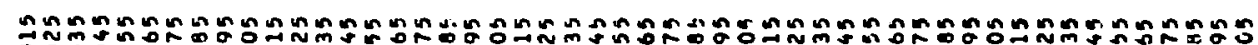

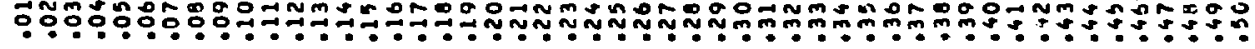


$\stackrel{x}{3}$

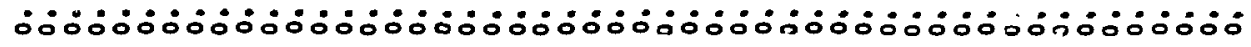

宽

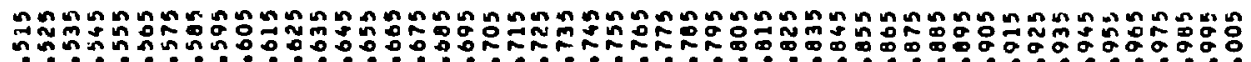

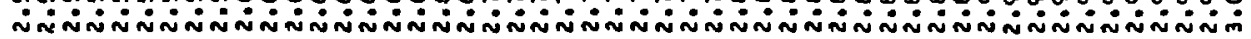

$\stackrel{\times}{\vec{u}}$

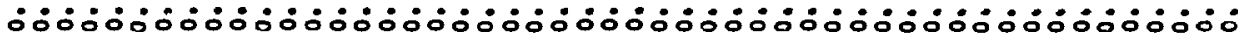

$\stackrel{5}{\stackrel{5}{*}}$

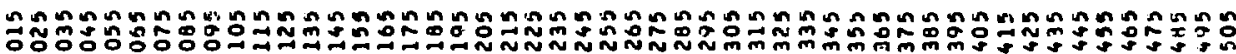

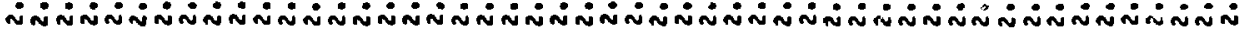

$\stackrel{3}{3}$

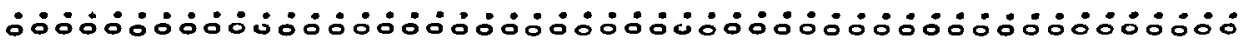

它

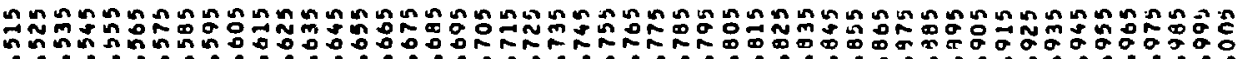

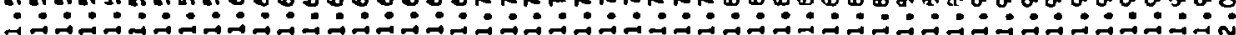

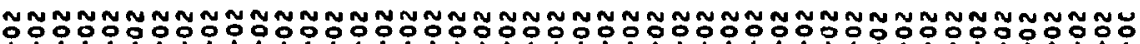

ipipipipipipipipipipipipipopipipipopopopopio

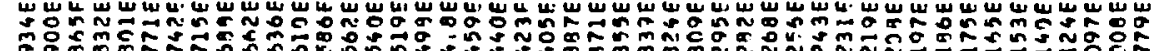

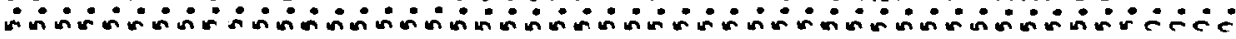

2

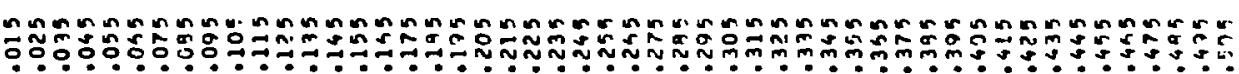

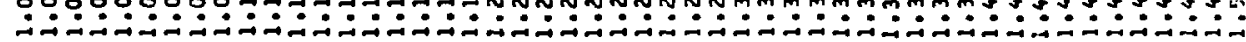

$\stackrel{x}{3}$

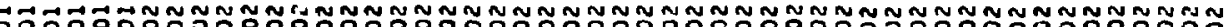

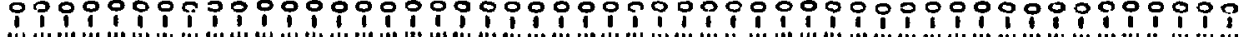

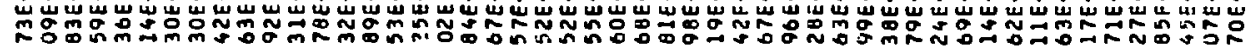

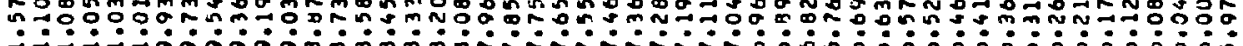

岩

委

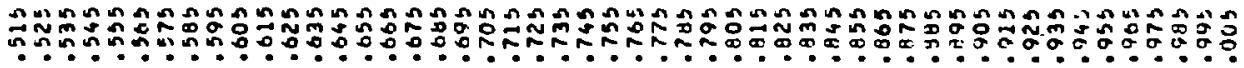

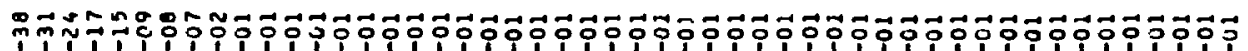

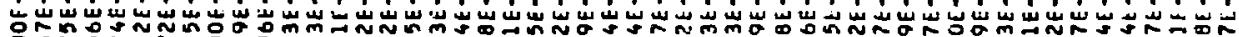
\$ôn

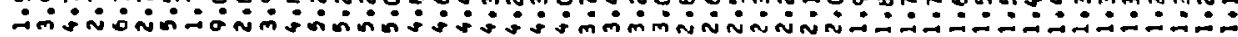

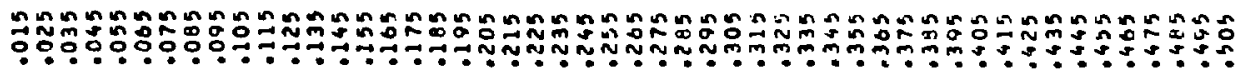


$\stackrel{\mathbf{x}}{\mathbf{3}}$

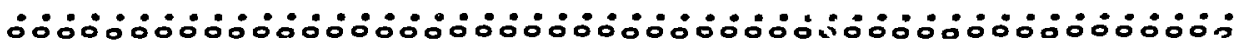

$\grave{z}$

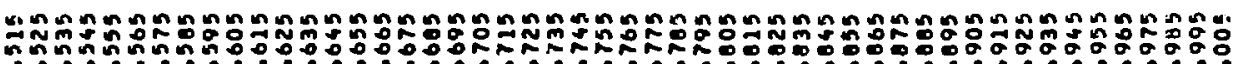

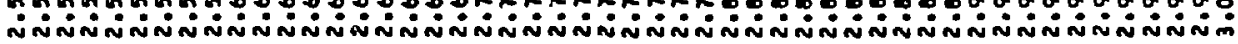

ב⿱艹

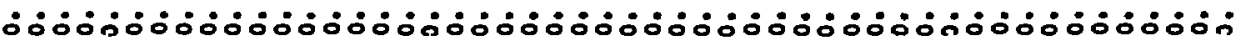

$\underset{\underline{z}}{\underline{z}}$

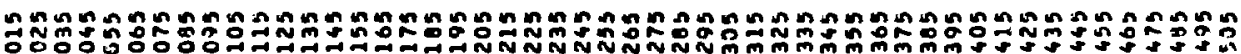

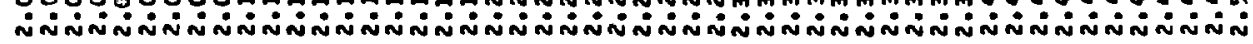

$\stackrel{x}{3}$

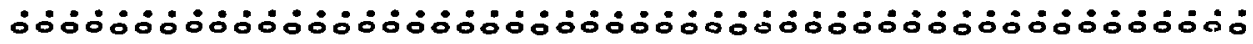

总

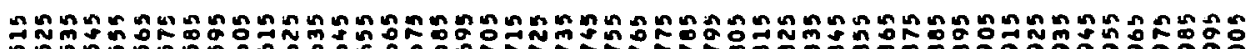
ก

\author{
岁
}

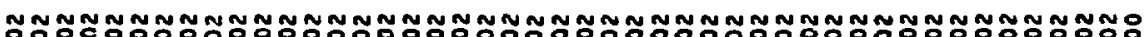

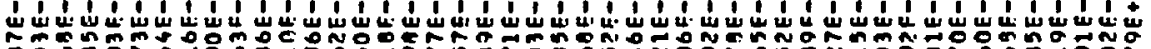

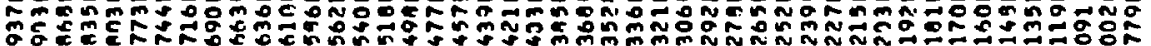

nெ

nnッn

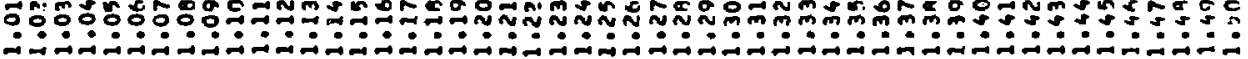

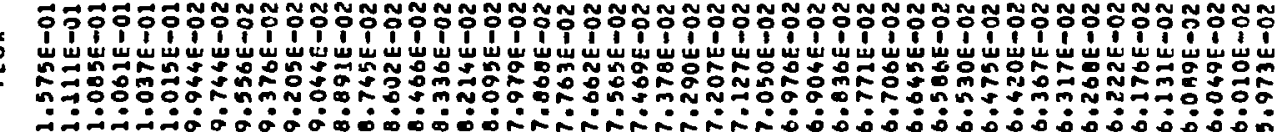

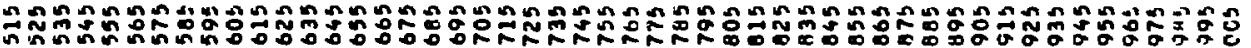

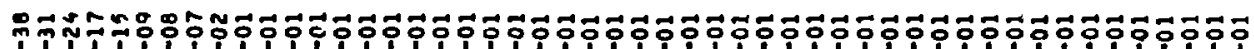

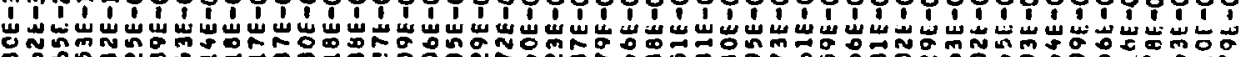

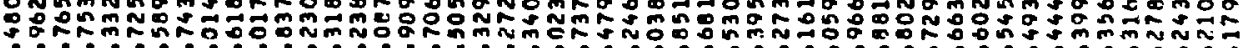

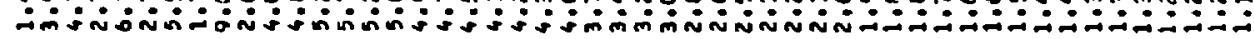


$\stackrel{x}{\mathbf{z}}$

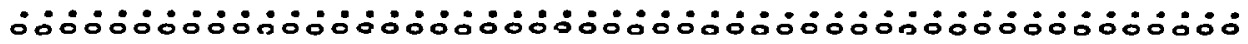

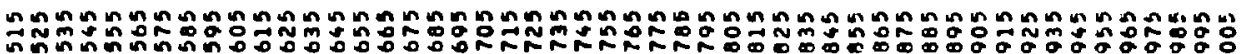

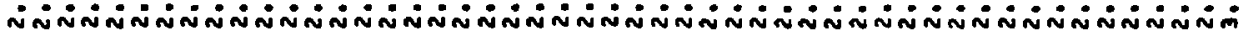

$\stackrel{x}{\underline{3}}$

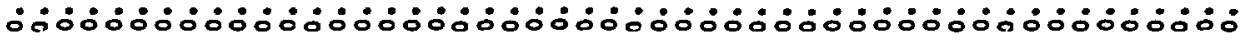

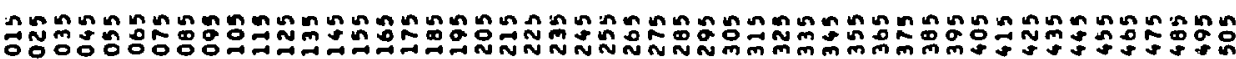

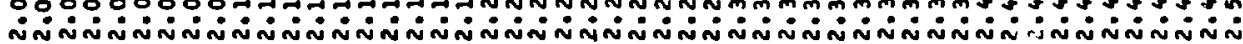

$\stackrel{x}{3}$

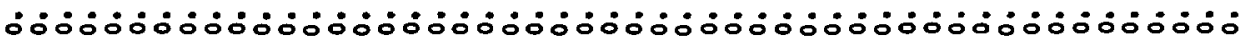

$\stackrel{2}{\stackrel{2}{*}}$

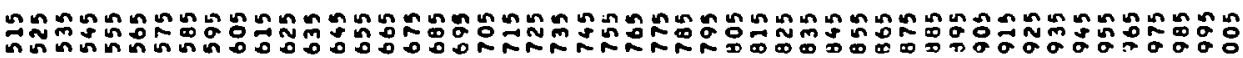

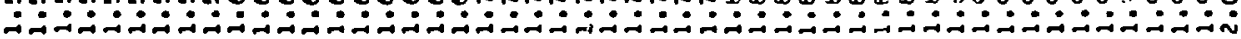

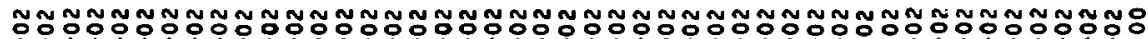

×

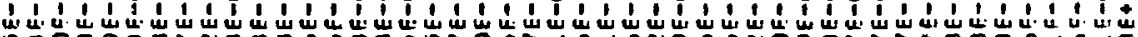

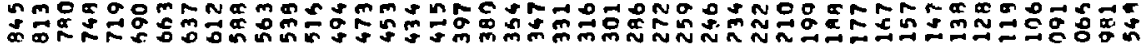

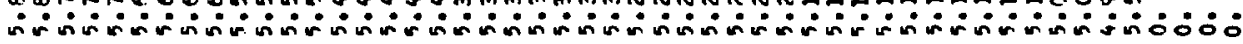

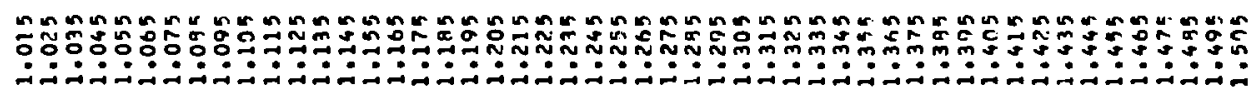

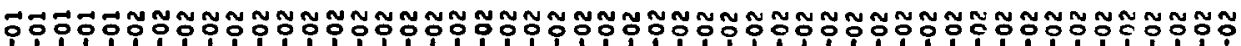

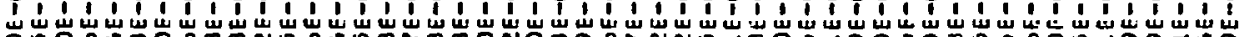

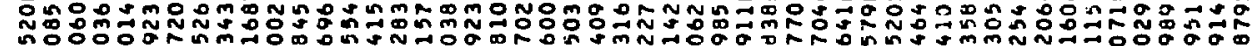

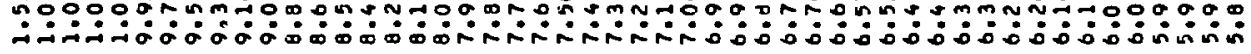

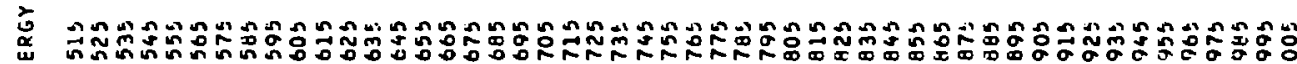

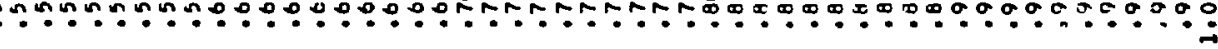

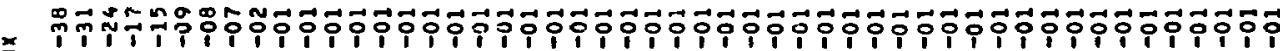

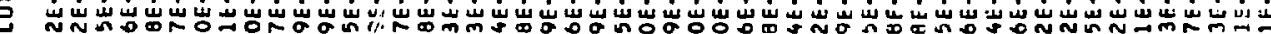

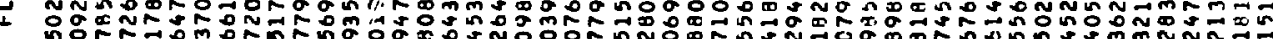

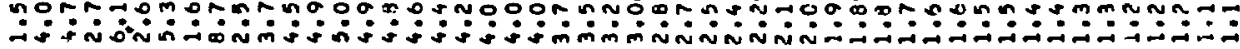

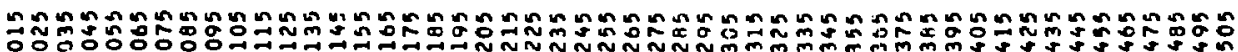

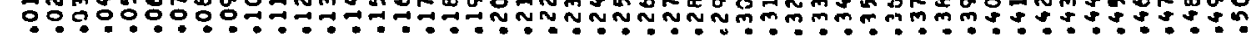


$\stackrel{x}{\vec{z}}$

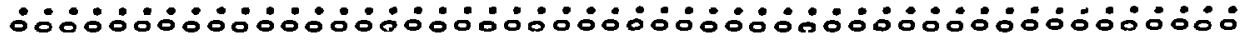

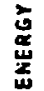

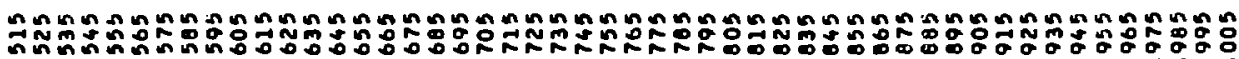

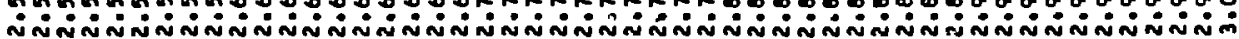

$\stackrel{\times}{3}$

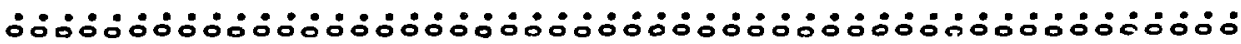

s.

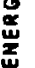

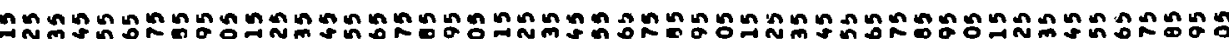

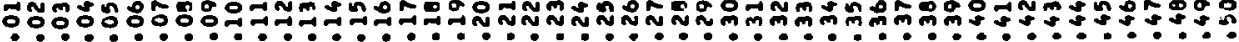

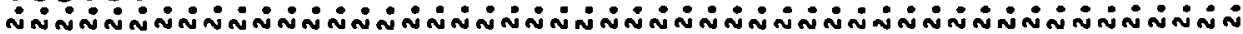

$\stackrel{\mathbf{x}}{\mathbf{3}}$

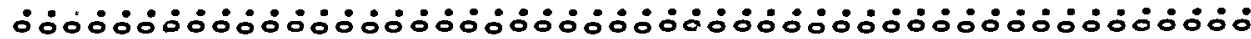

to

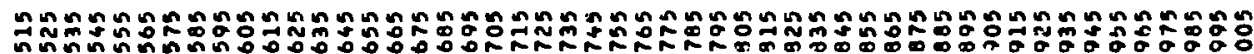

垈

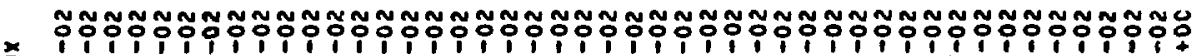

五

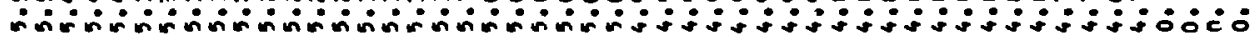

2

nמn

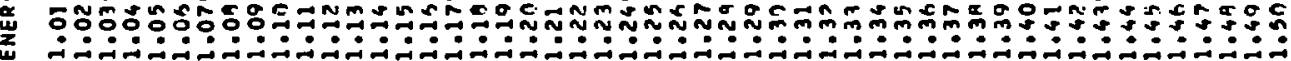

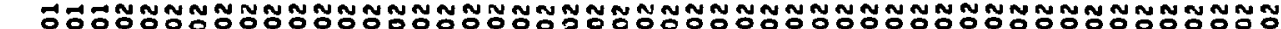

$\stackrel{x}{3}$

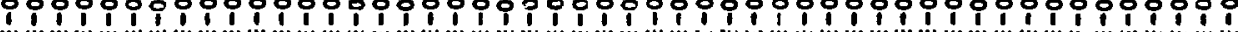

u

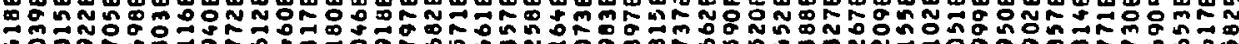
Ta

อ

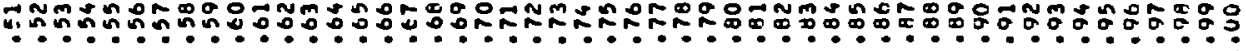

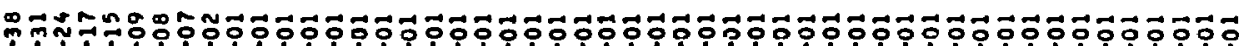

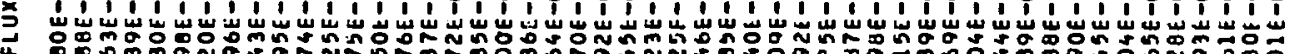

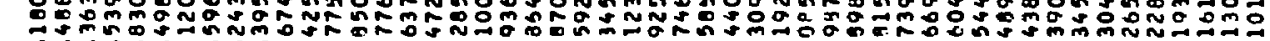

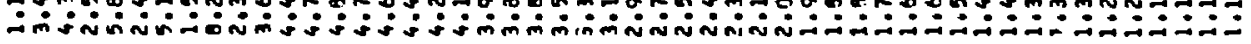

焉

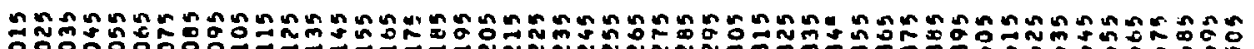

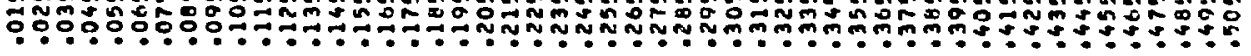


$\stackrel{x}{3}$

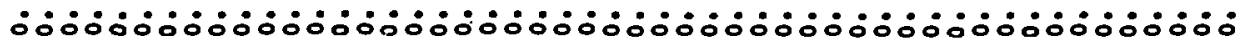

它

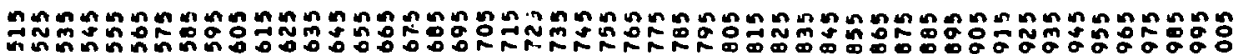

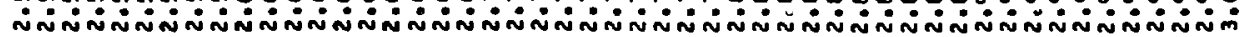

$\stackrel{\times}{3}$

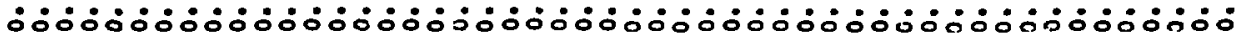

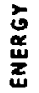

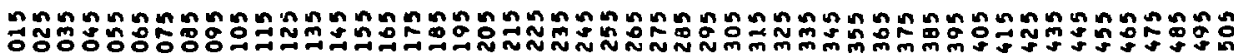

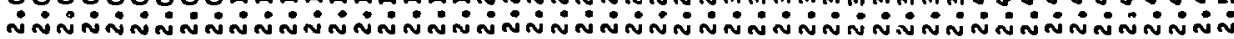

$\stackrel{3}{3}$

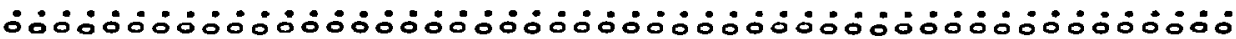

$\stackrel{5}{2}$

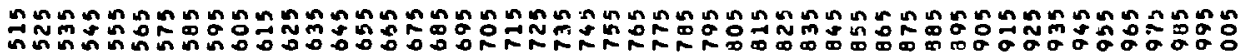

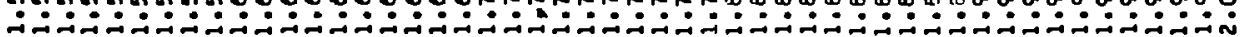

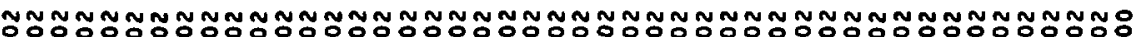

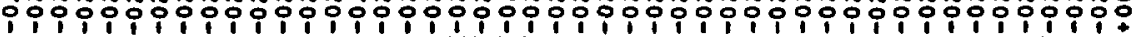

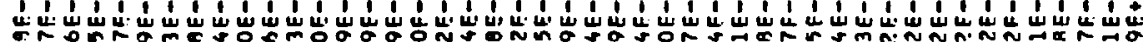

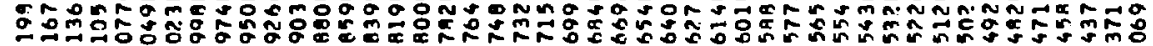

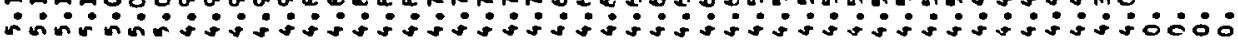

2

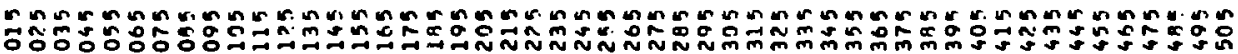

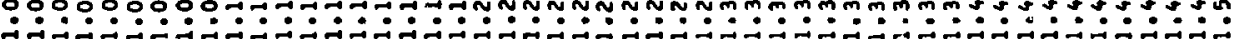

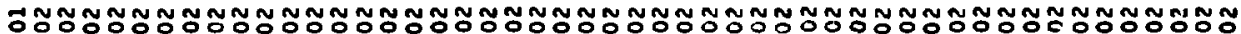

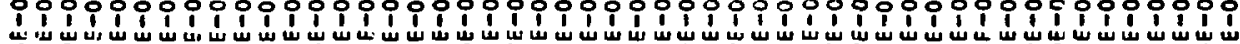

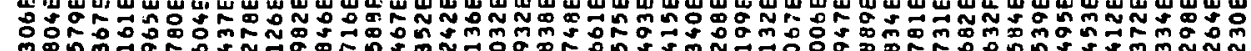

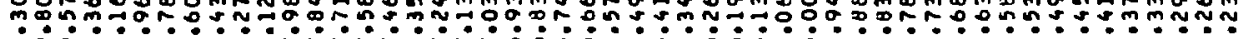

s.

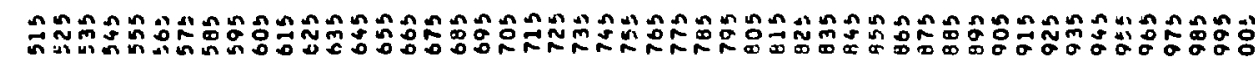

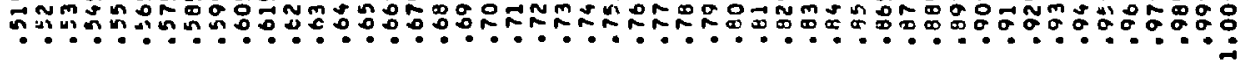

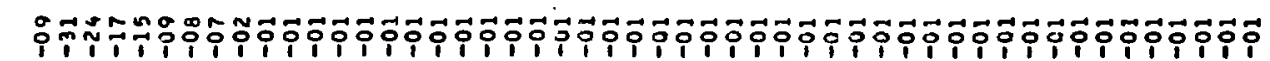

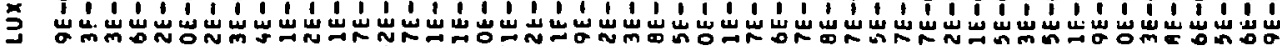

屯

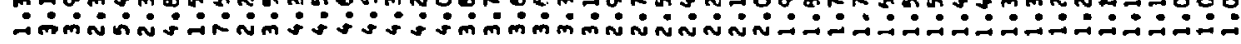

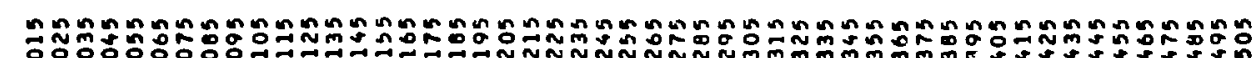

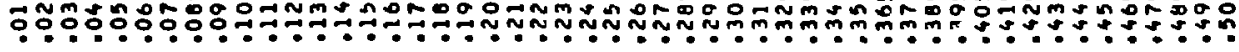




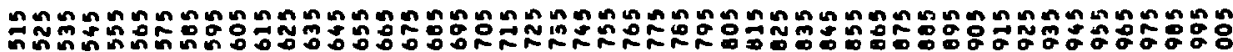

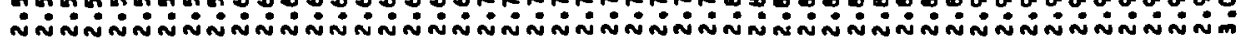

$\stackrel{x}{3}$

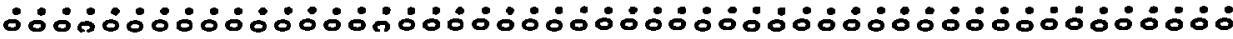

总

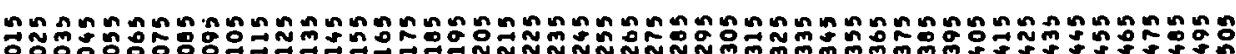

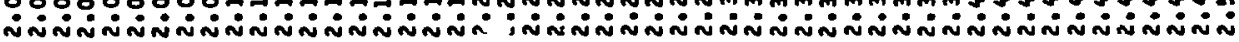

$\stackrel{5}{3}$

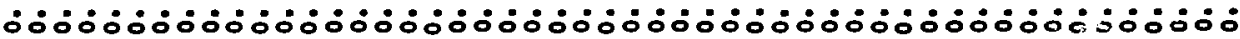

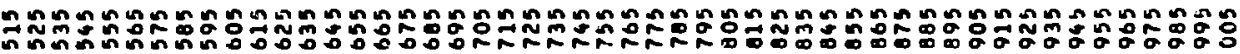

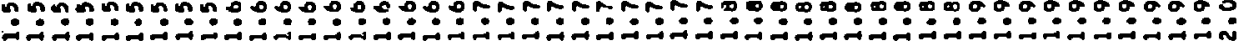

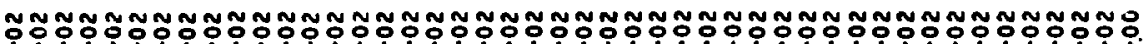

pippipipipipipipipipipippopipipipipipipo

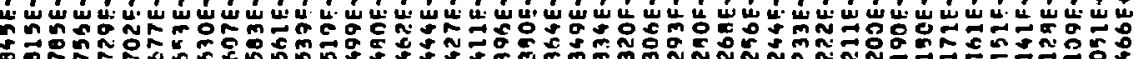

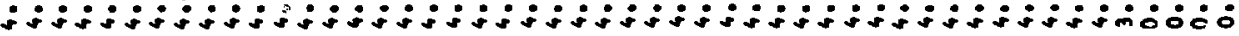

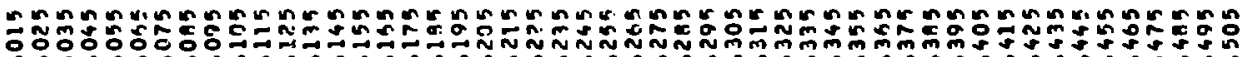

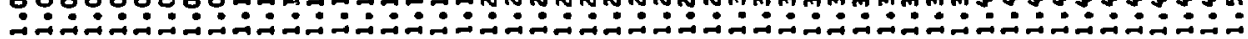

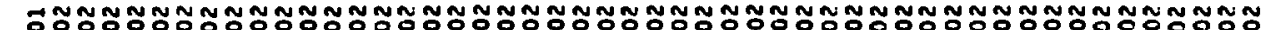

ipipipipipipipipipipipipipipopipopipipipipo

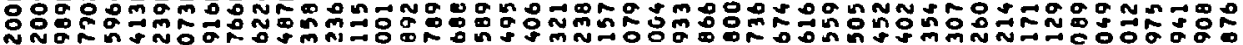

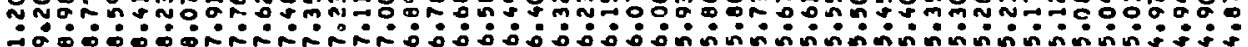

ל

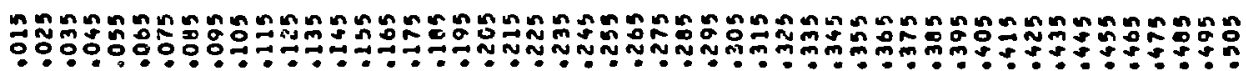


$\stackrel{x}{3}$

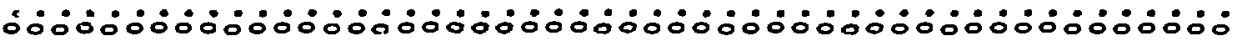

$\stackrel{2}{\stackrel{2}{2}}$

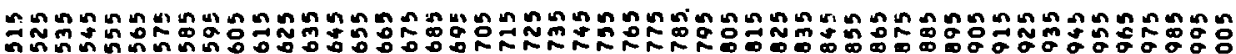

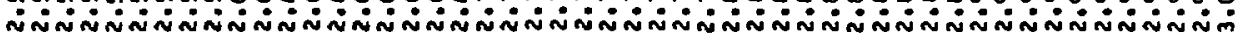

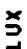

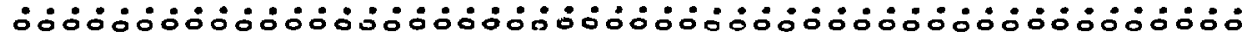

点

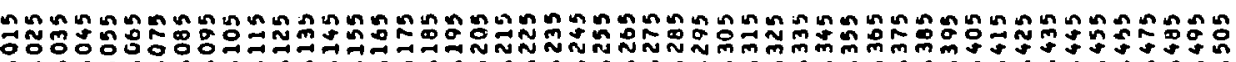

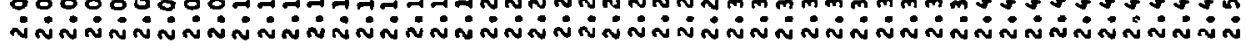

$\stackrel{x}{3}$

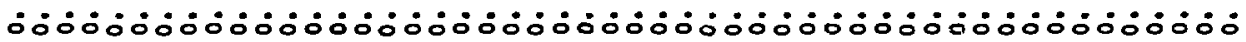

惫

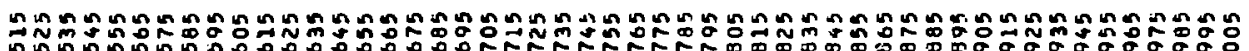

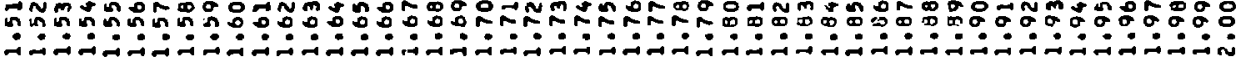

\section{$\stackrel{\times}{\mathbf{u}}$}

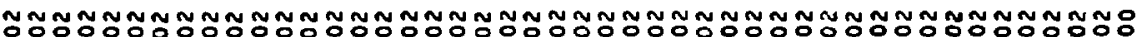

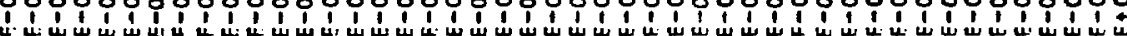

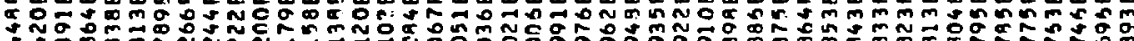

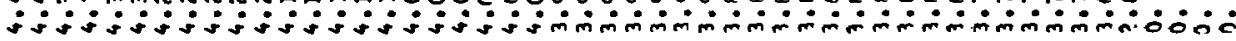

总

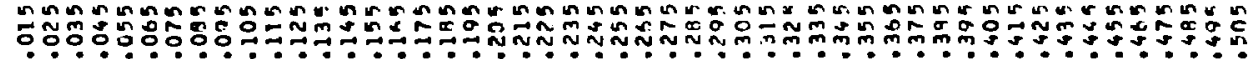

2

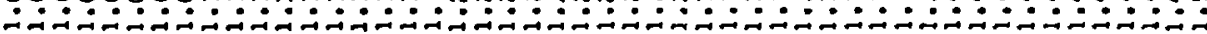

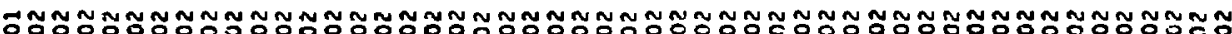
ipipipipipipipipipipipipipipipipip

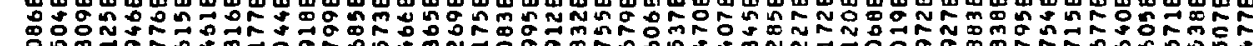
ะ

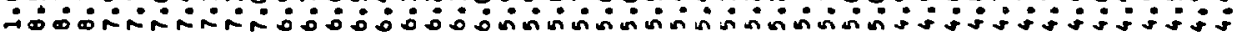

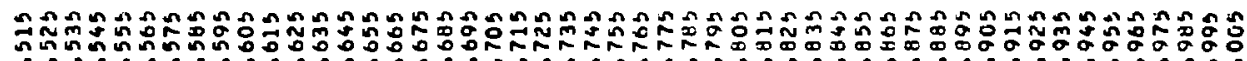
กำกำ.

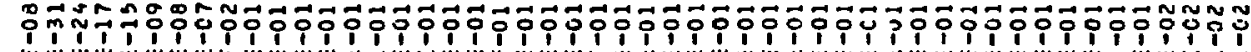

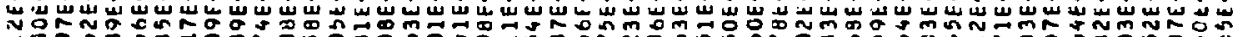

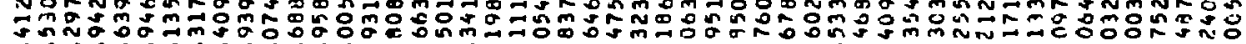

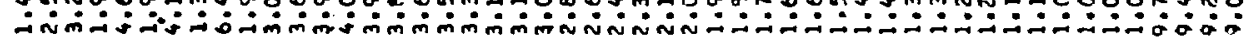

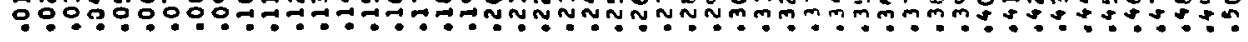


$\underset{\mathbf{3}}{\mathbf{3}}$

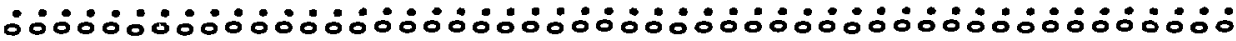

察

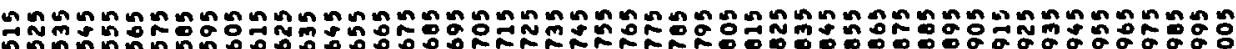

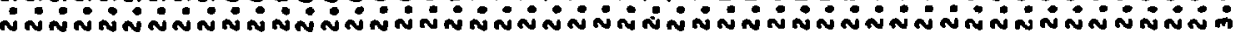

$\underset{3}{\stackrel{x}{u}}$

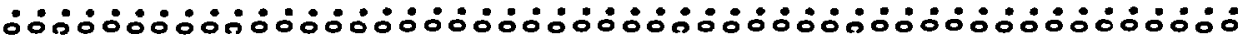

3

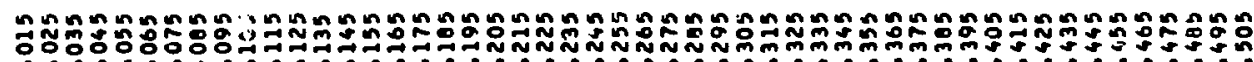

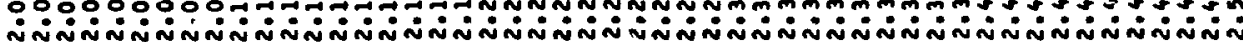

$\stackrel{\text { x }}{\mathbf{z}}$

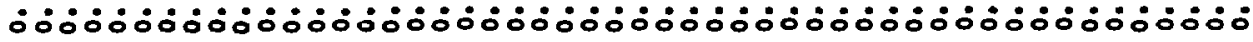

3

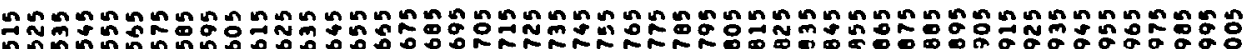

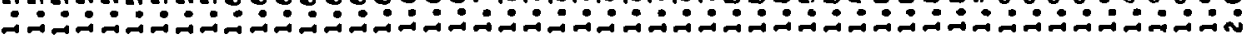

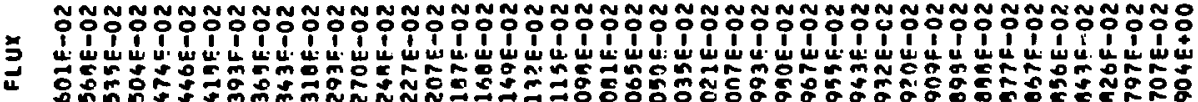
núr.

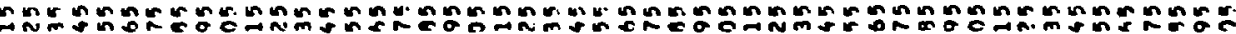

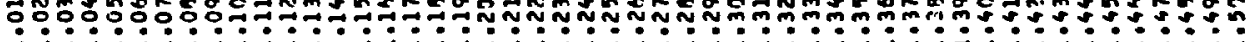

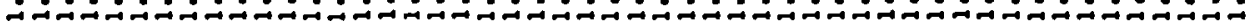

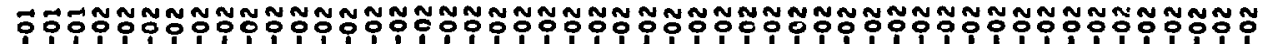

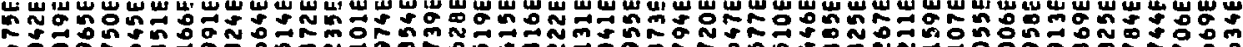

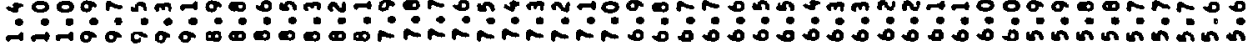

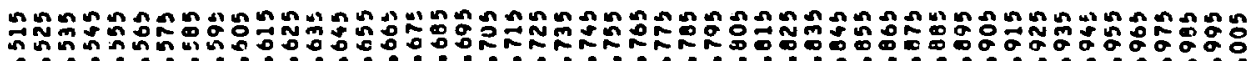

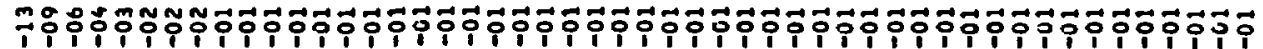

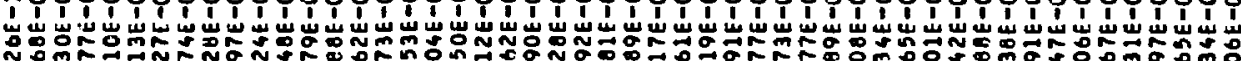

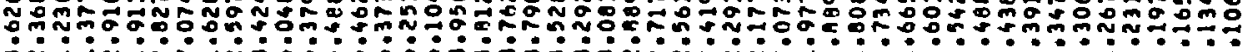

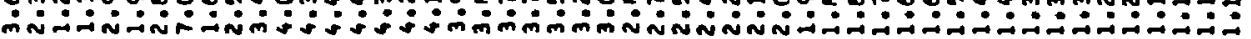

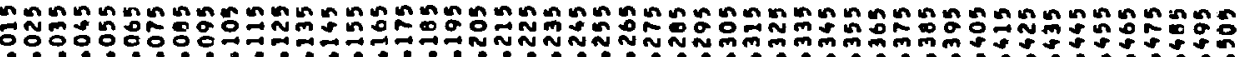


$\stackrel{x}{3}$

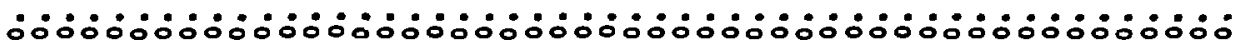

学

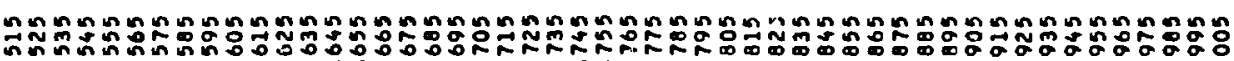

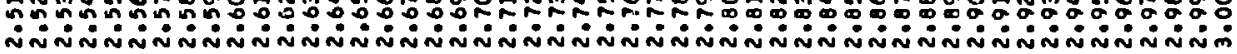

$\stackrel{x}{\underline{3}}$

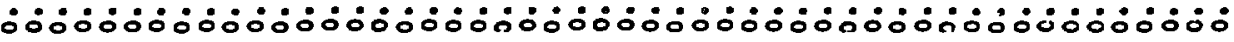

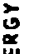

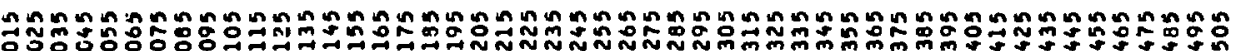

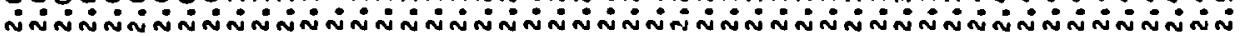

$\stackrel{x}{3}$

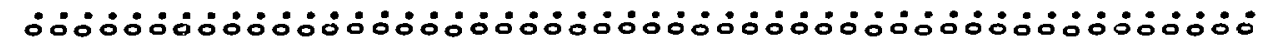

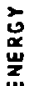

7

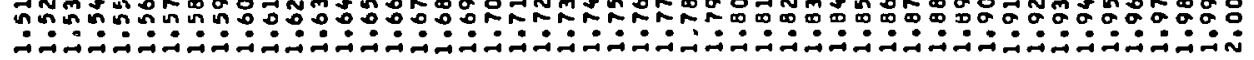

茨

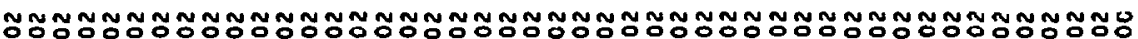

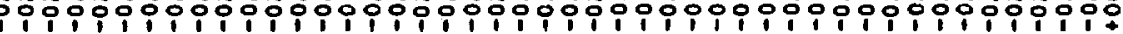

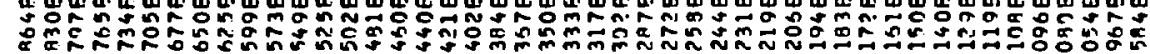

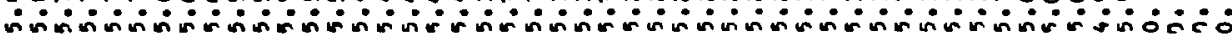

¿

崖

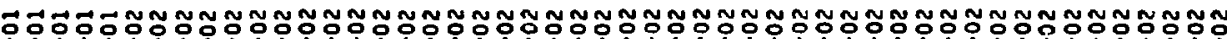

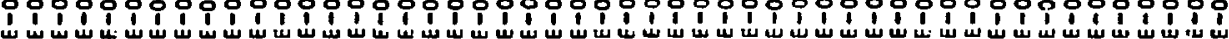

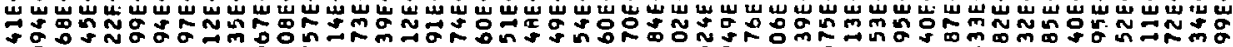

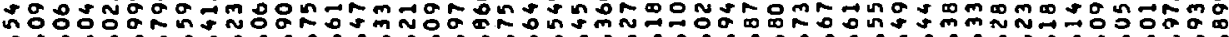

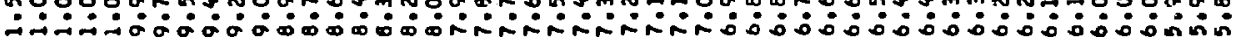

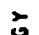

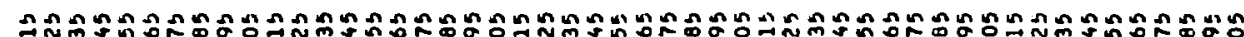

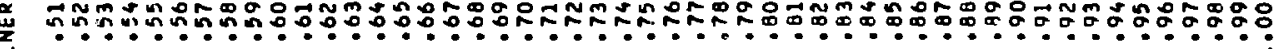

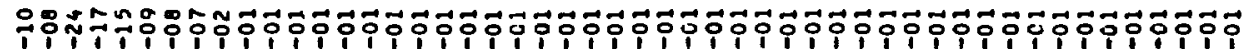

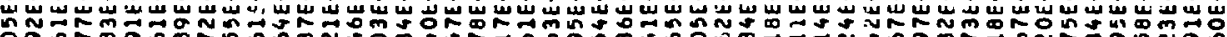

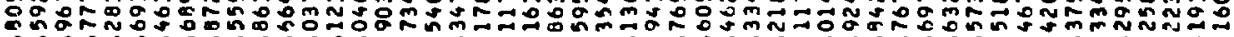

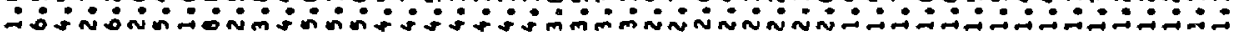

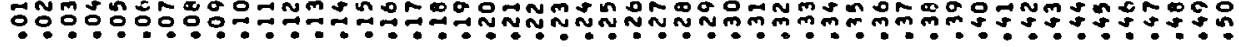


క̦

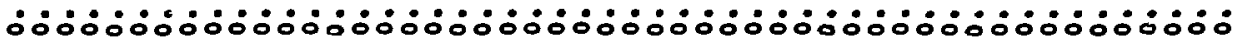

莡

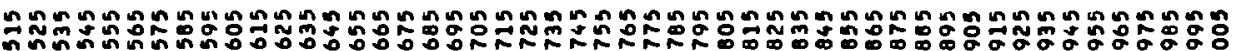

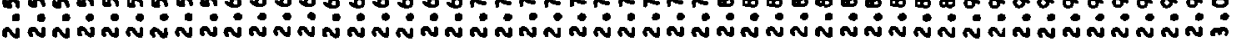

岌

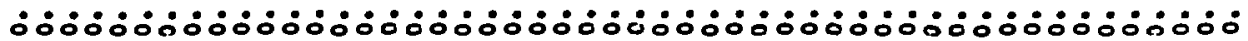

产

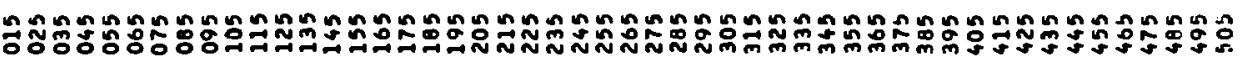

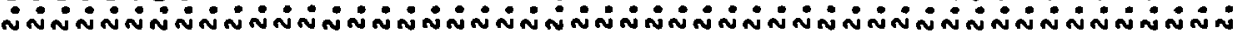

క

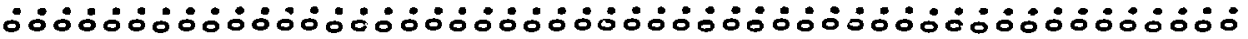

竧

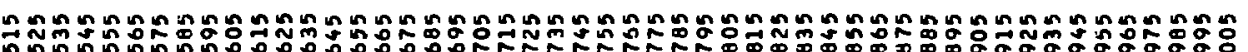

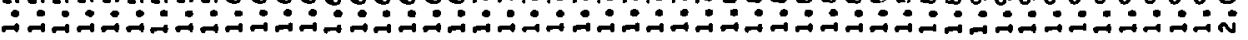

\section{$\stackrel{4}{3}$}

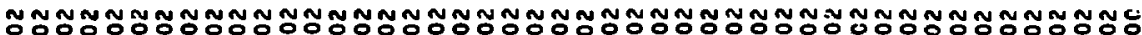

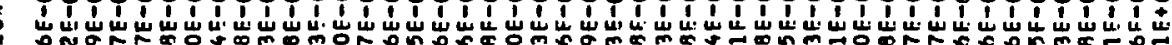

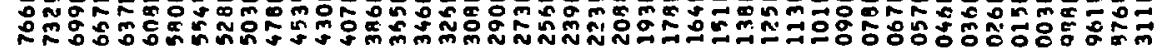

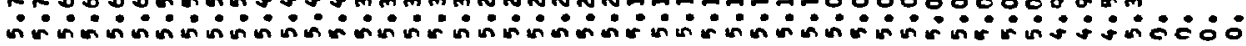

它

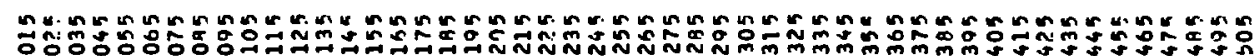

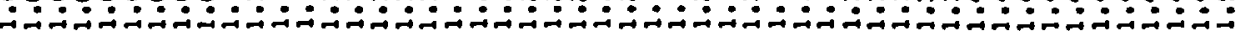

\section{节}

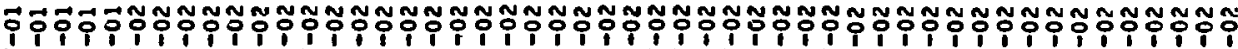

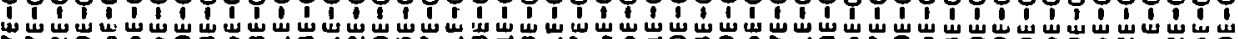

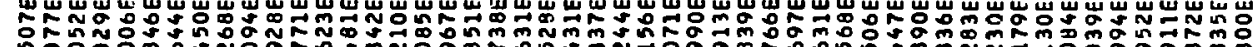

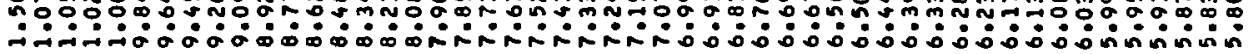

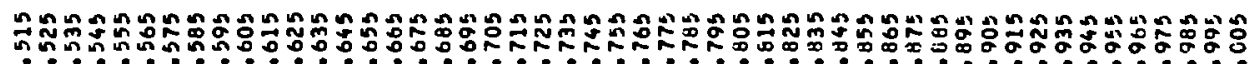

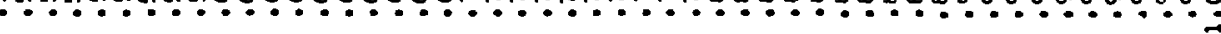

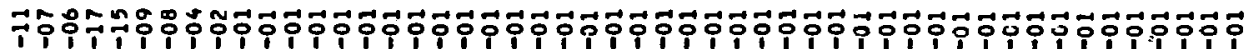

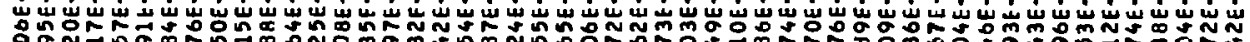

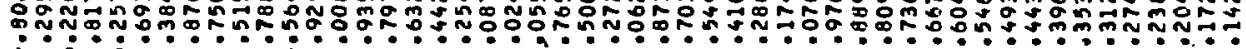

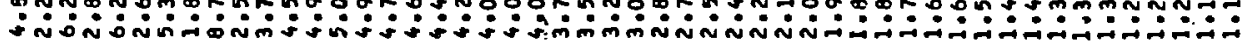

nณnn

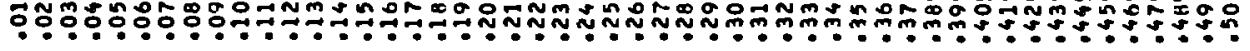




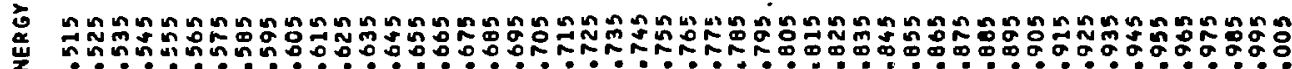

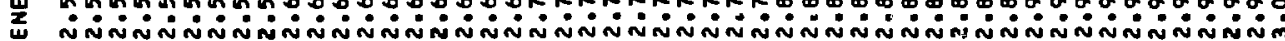

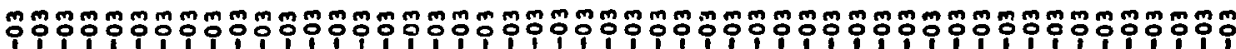

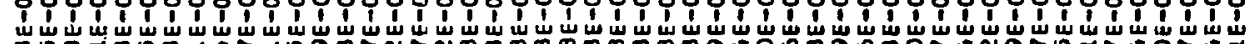

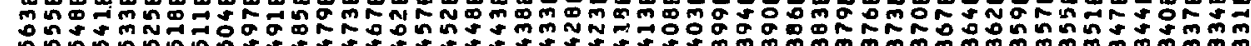

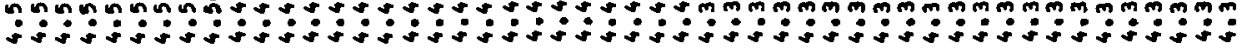

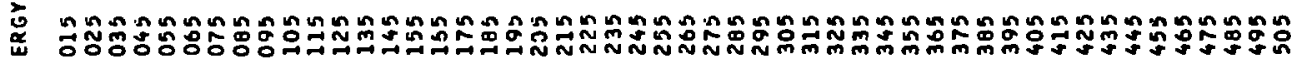
z

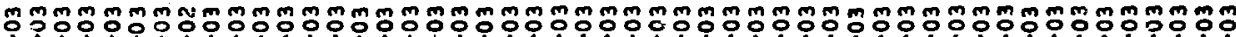

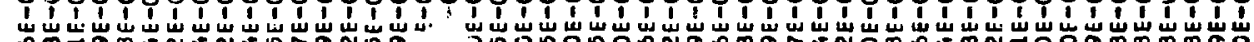

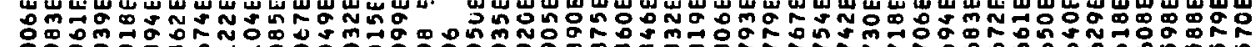

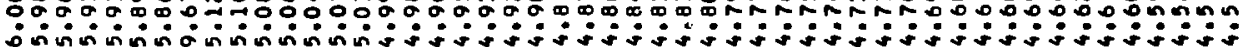

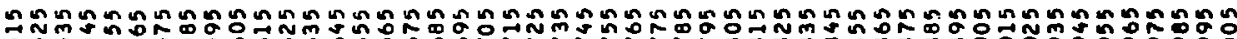

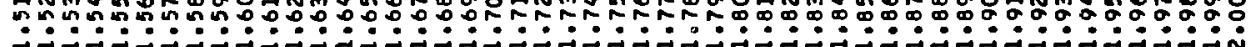

mำmำmmmmmmmmmmmmmmmmmmmmmmmmmmmmmmmmmmmmmmmmmm

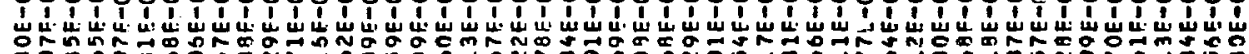

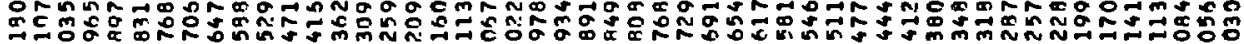

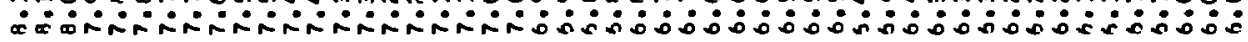

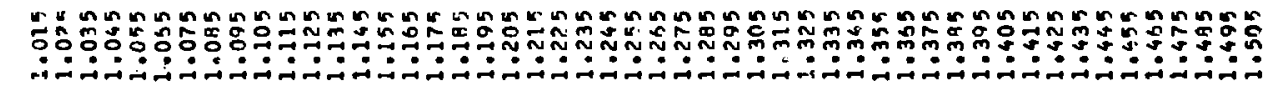

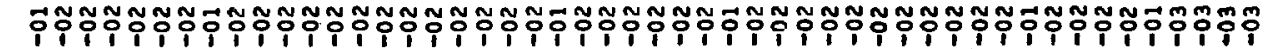

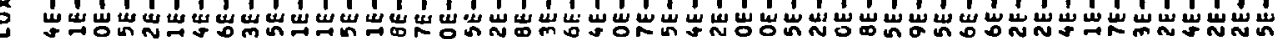

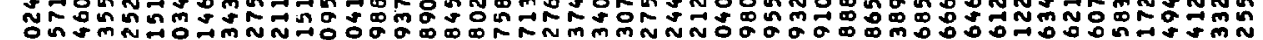
í: לे⿺

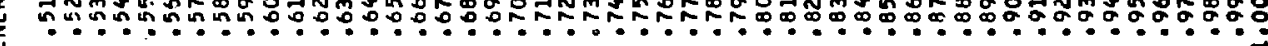

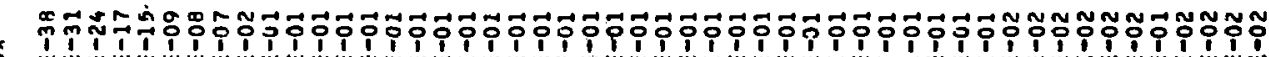

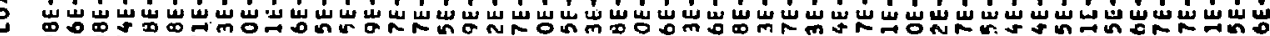

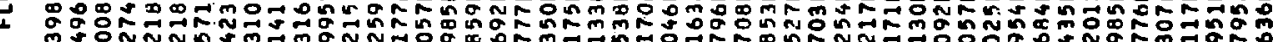

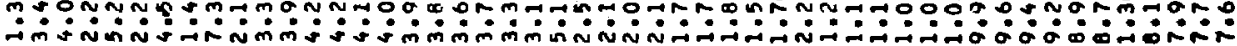




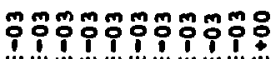

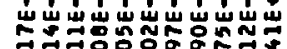

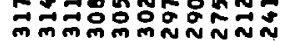

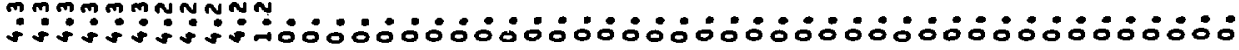

उे

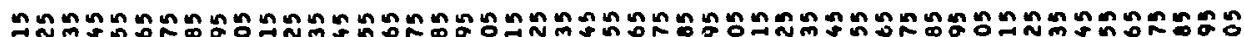

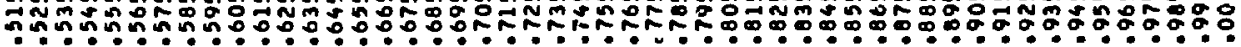

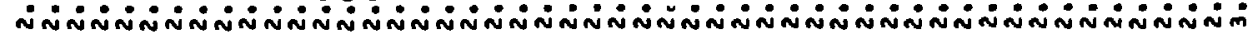

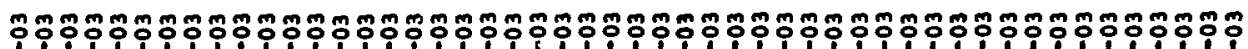

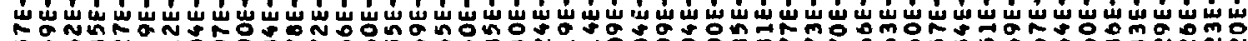

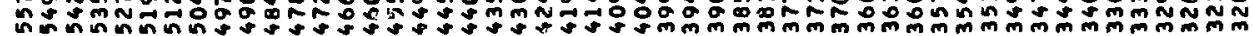

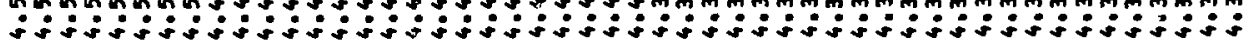

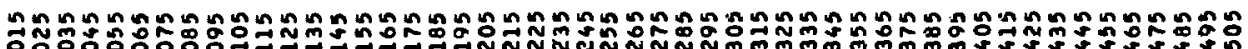

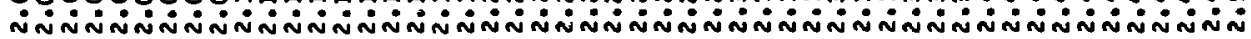

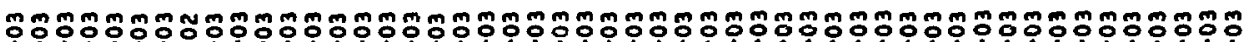

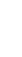

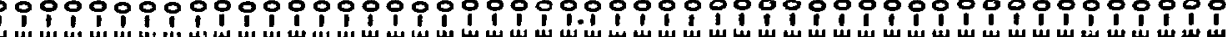

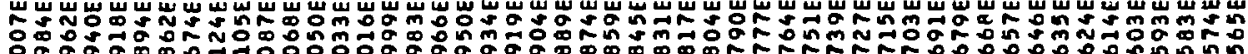

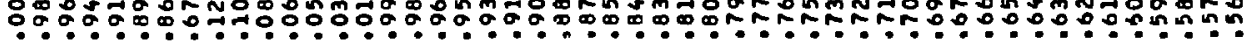

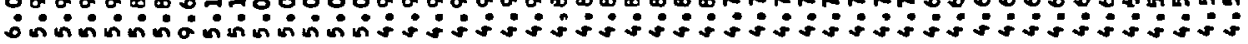

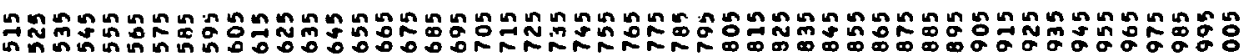

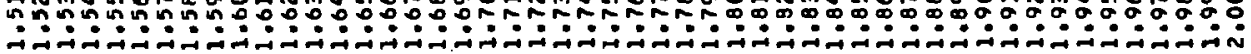

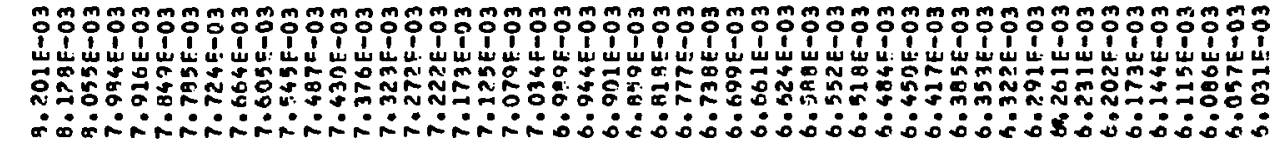

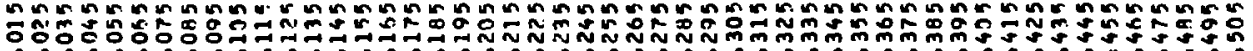

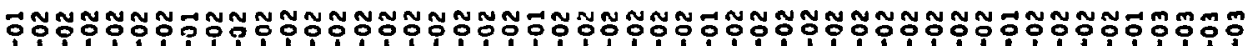

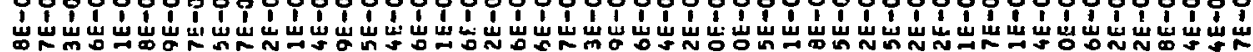

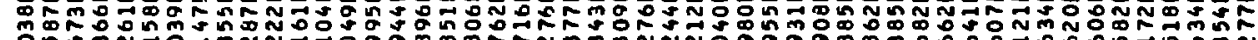

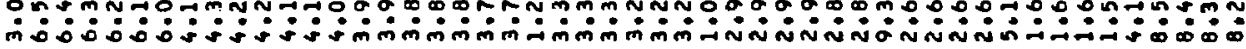

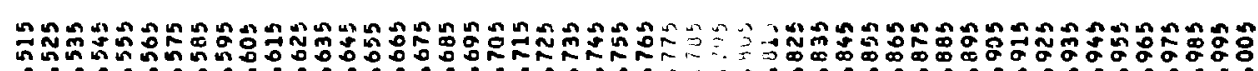

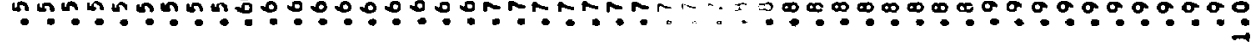

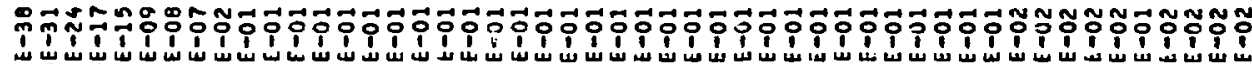

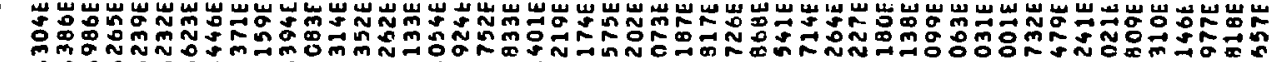

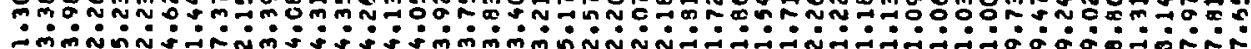

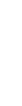

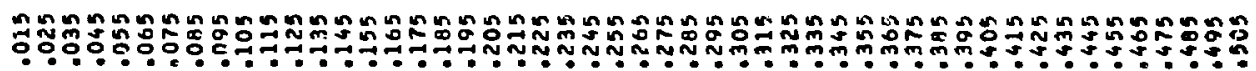




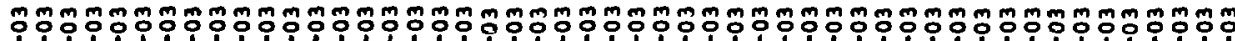

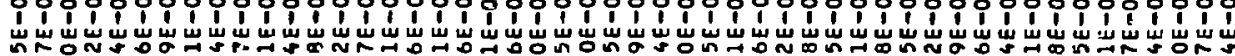

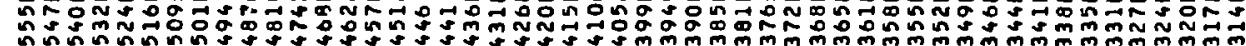

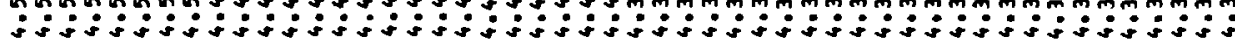

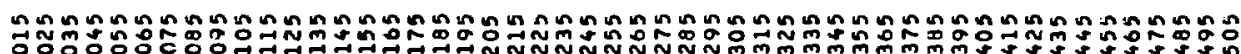

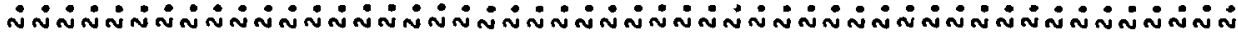

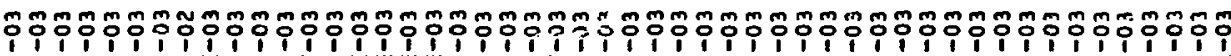

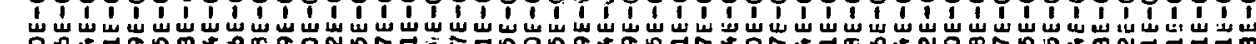

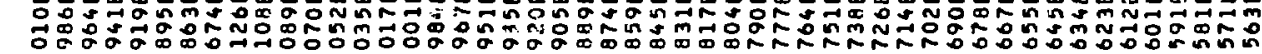

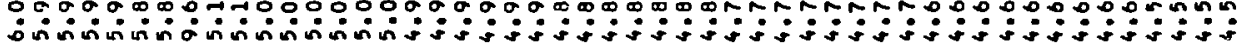

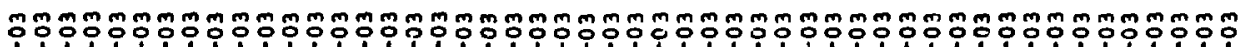

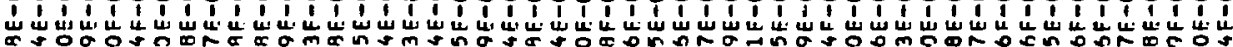

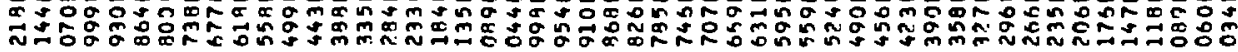

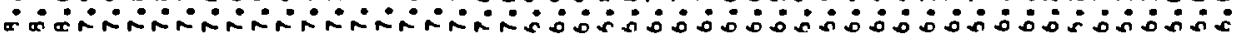

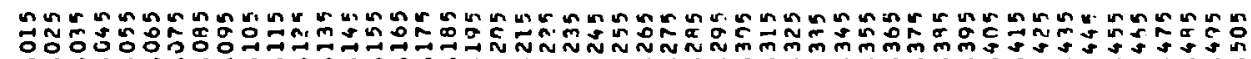

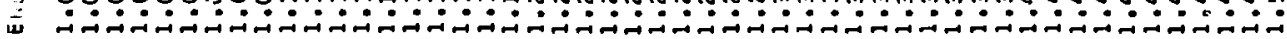

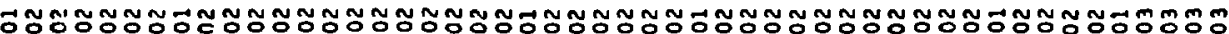

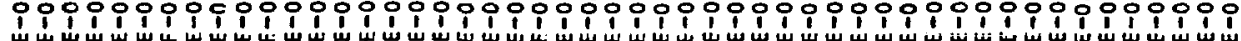

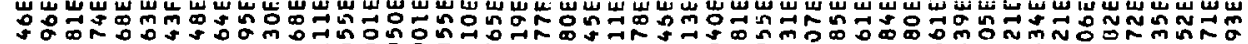
Oํํำ

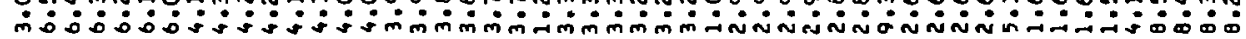

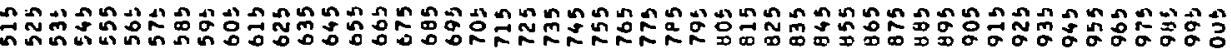
×

马

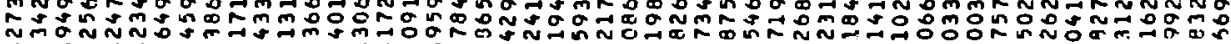

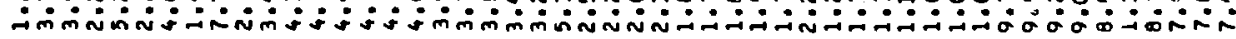

2

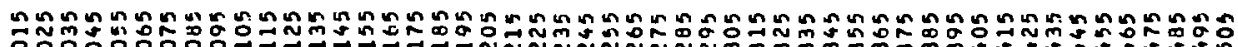

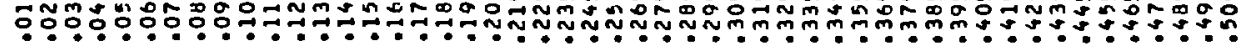




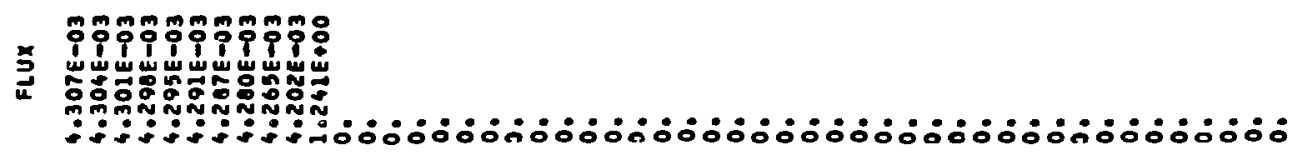

$\frac{\mathrm{z}}{2}$

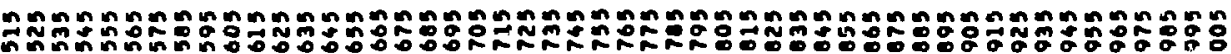

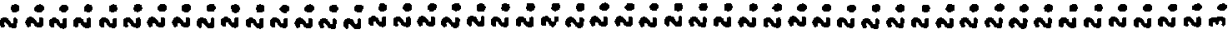

3

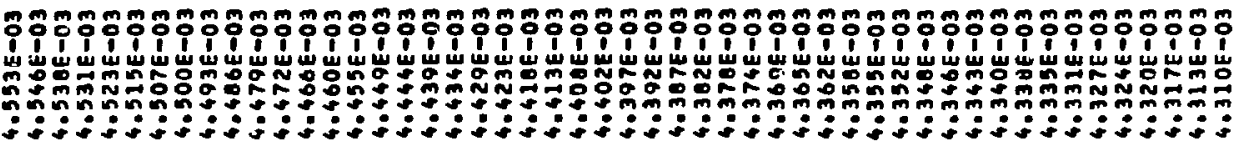

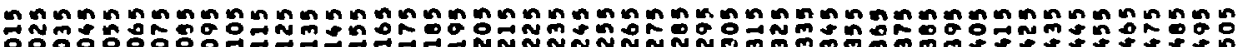

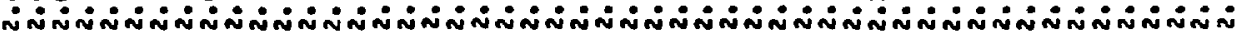

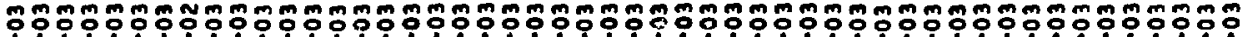

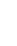

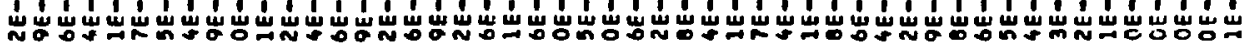

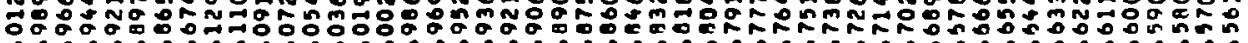

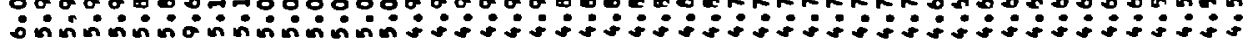

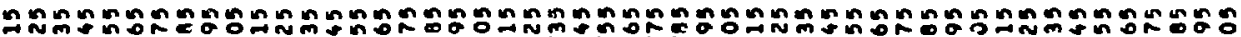

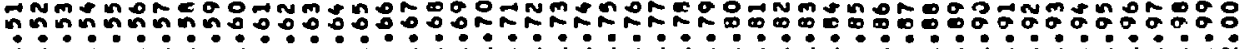

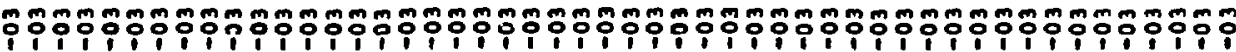

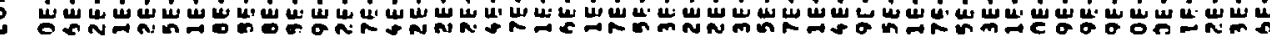

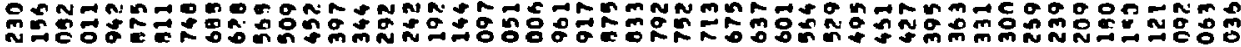

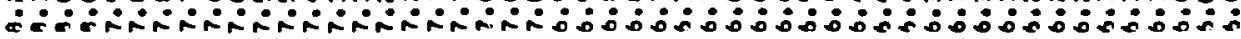

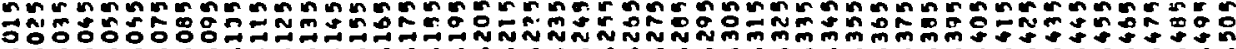

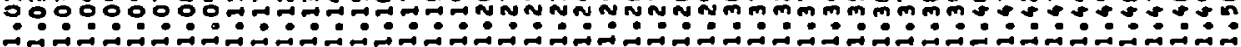

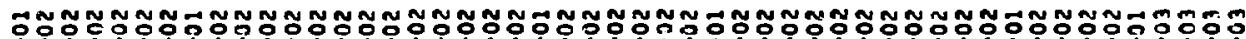

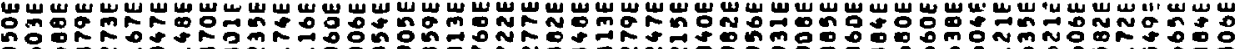

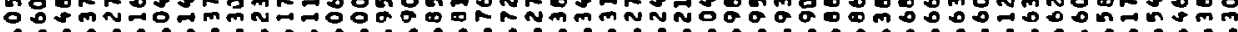
î

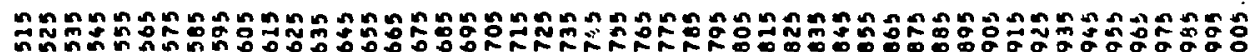

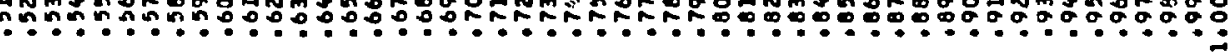

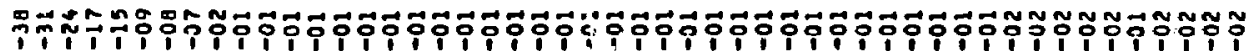

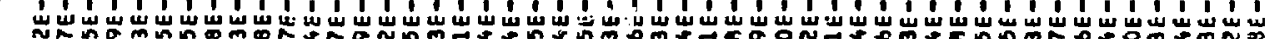

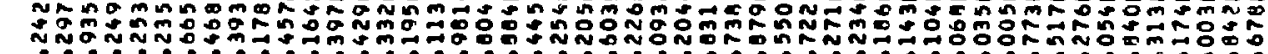

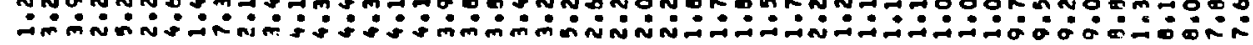

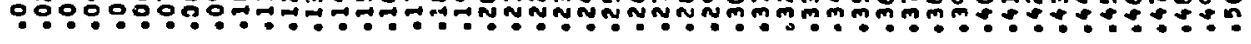




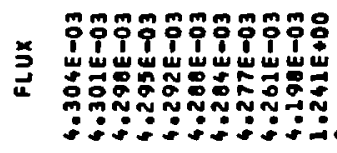

莬

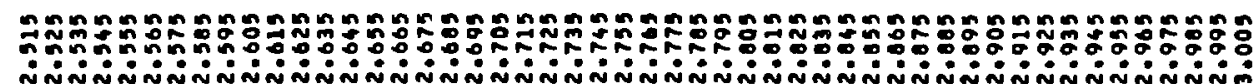

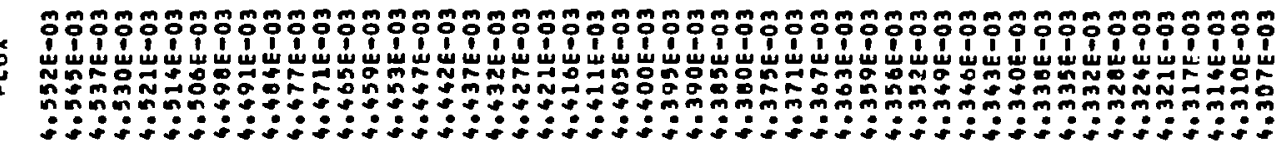

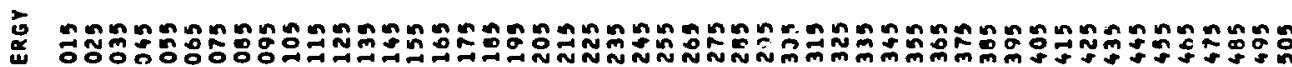

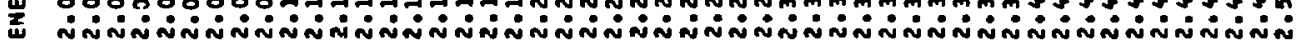

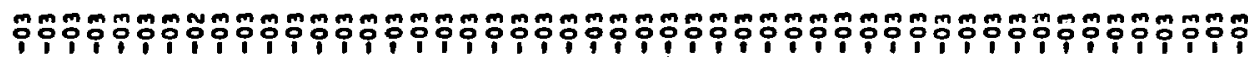

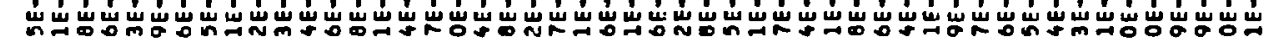

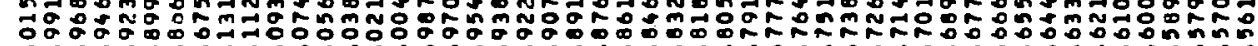
onvinn

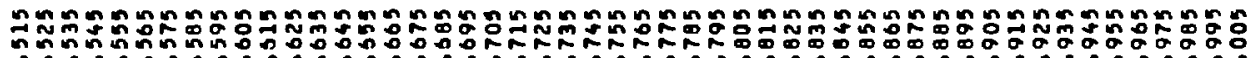

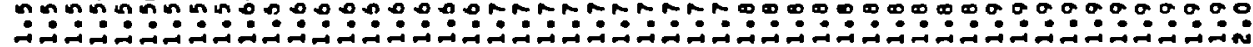

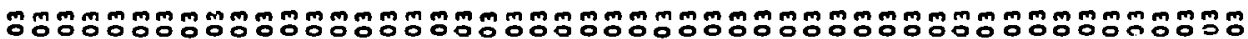

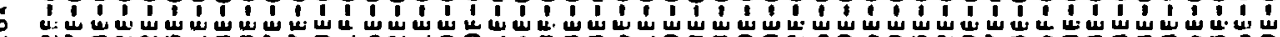

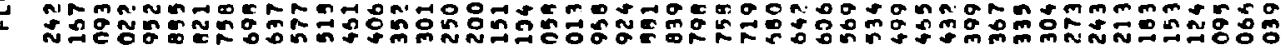

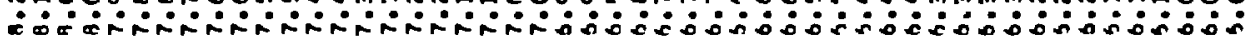

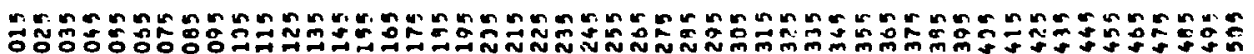

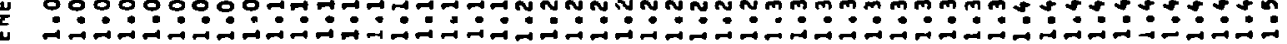

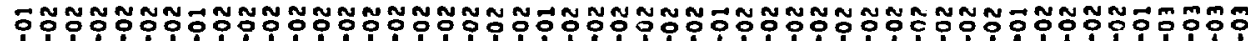

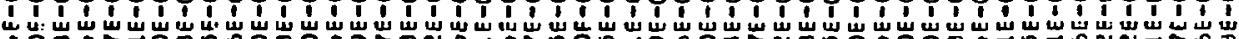

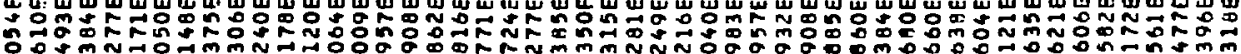
mo:

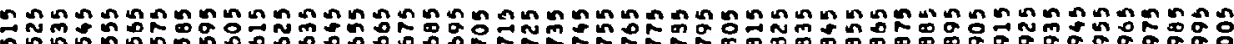

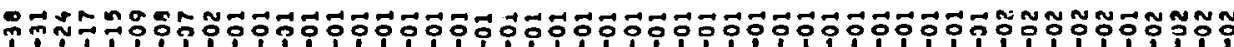

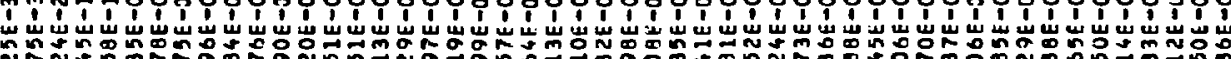
N NNN N

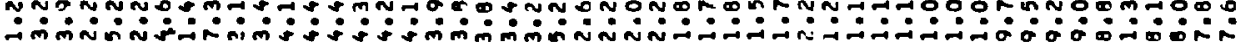

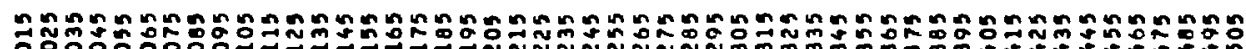

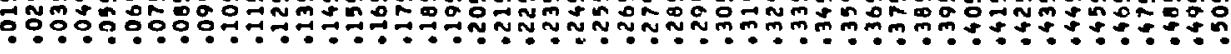




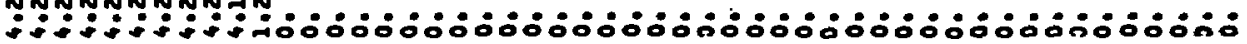

2n ñ்

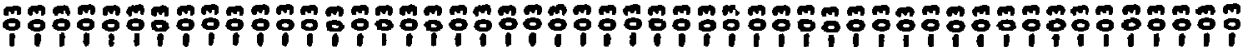

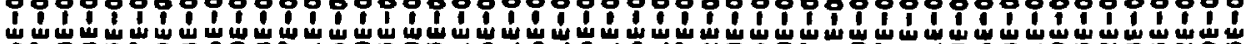

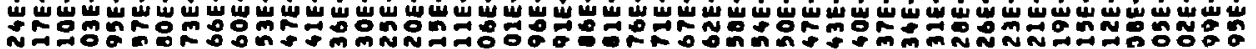

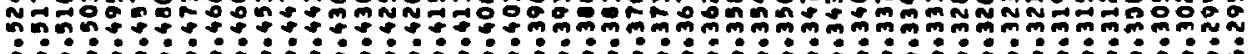

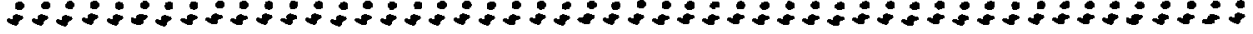

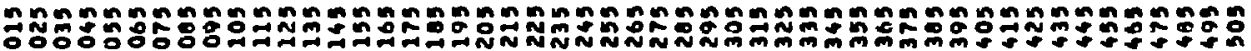

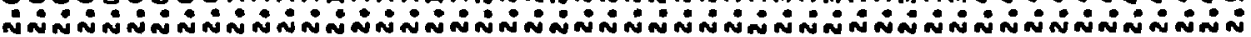

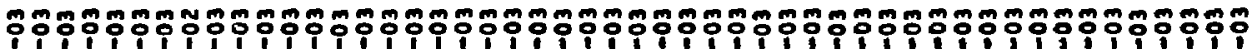

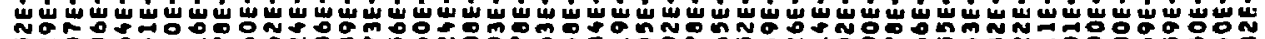

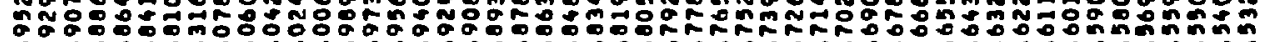
ń

3

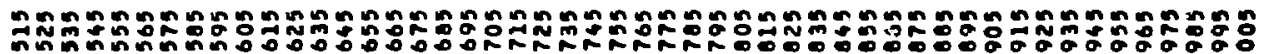
:ด̆

mºñmmmm

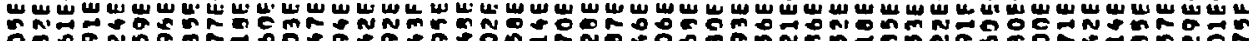

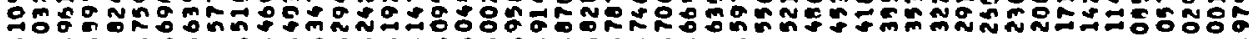

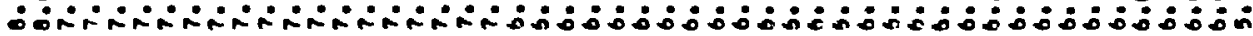

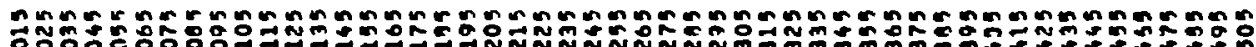

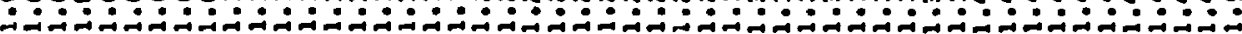

\section{$\stackrel{3}{3}$

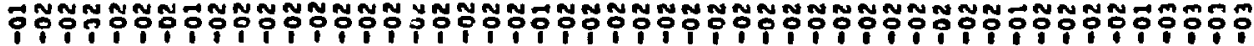

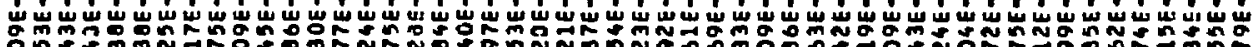

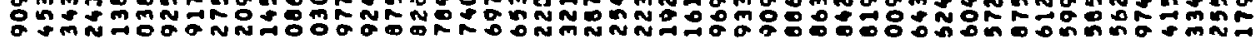

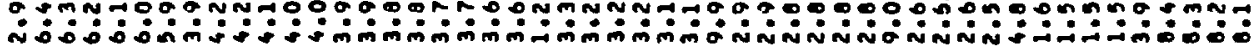

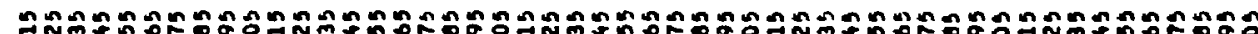

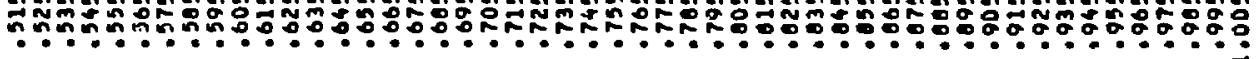

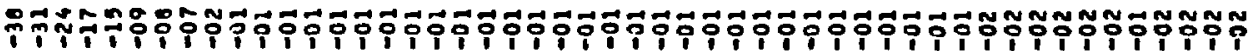

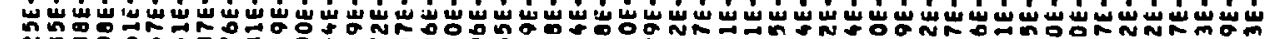

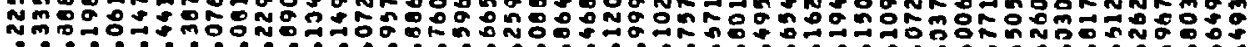

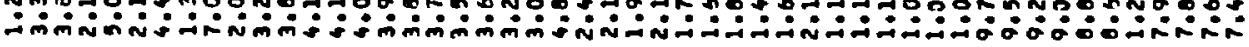

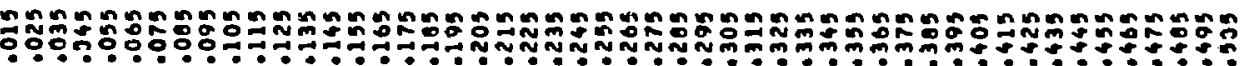




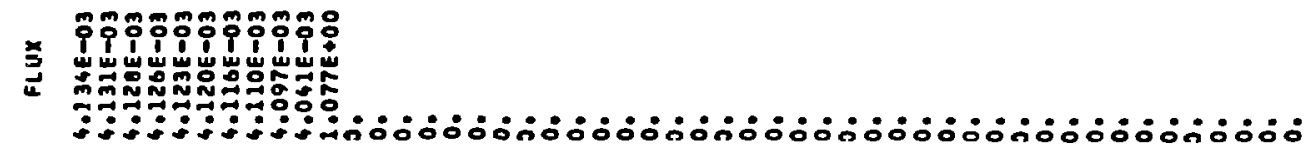

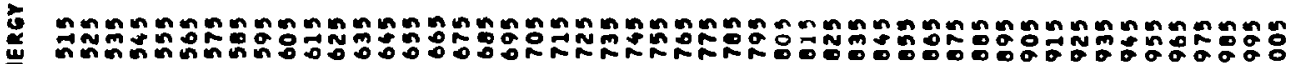

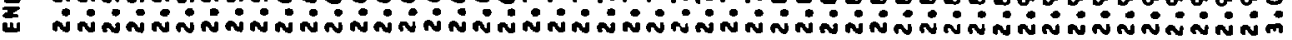

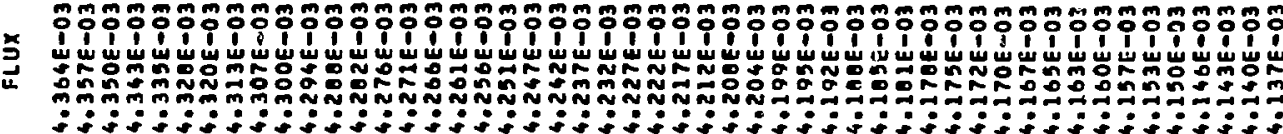

ô

U N

苟

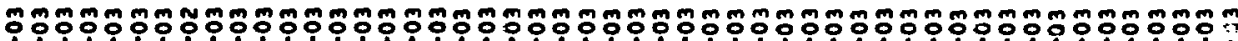

要

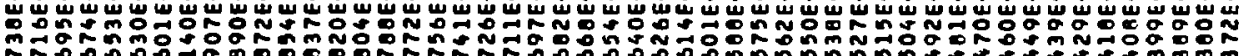

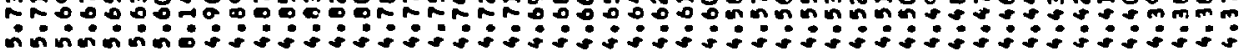

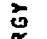

$\underset{w}{m}$

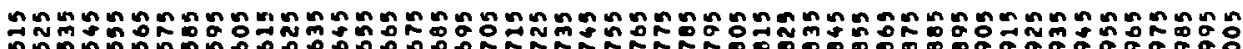

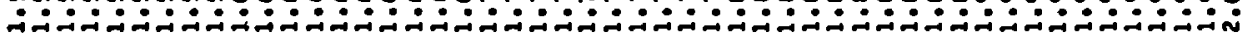

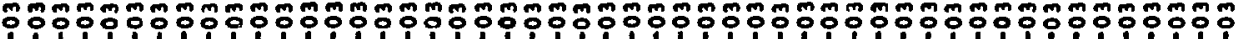

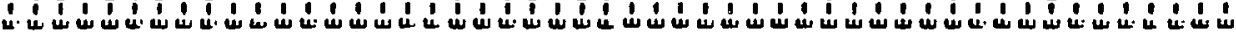

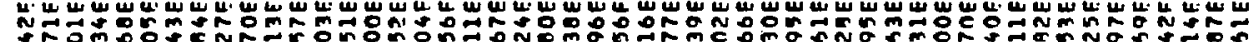

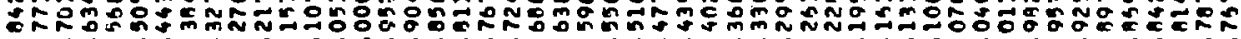

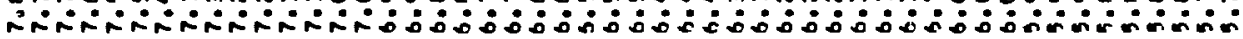

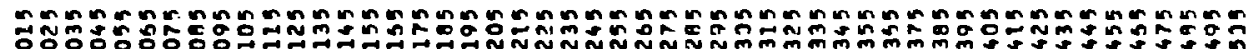

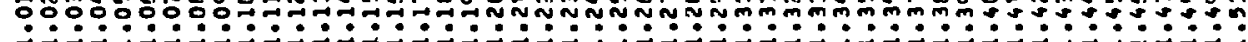

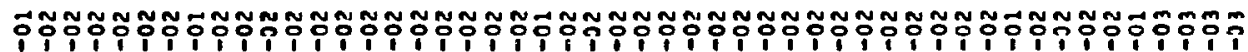

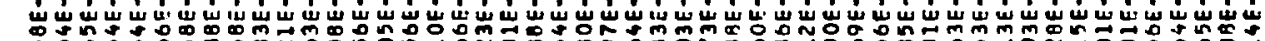

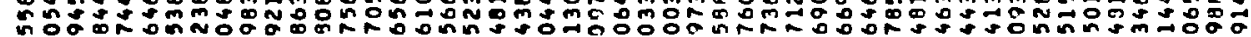

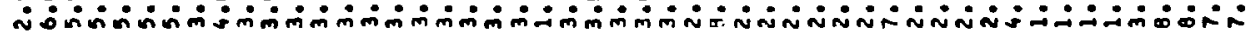

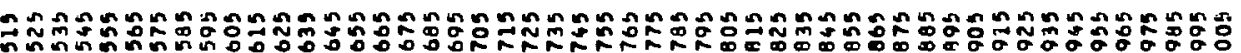

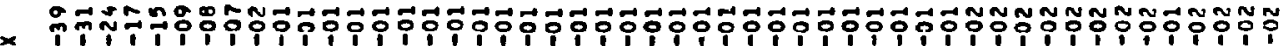

× أُ

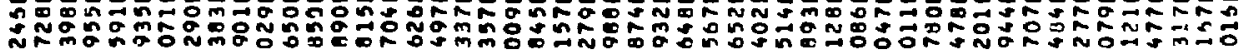

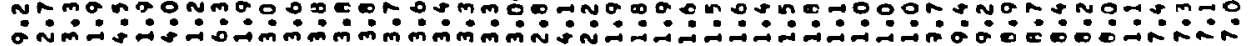

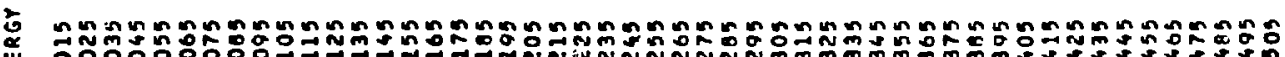

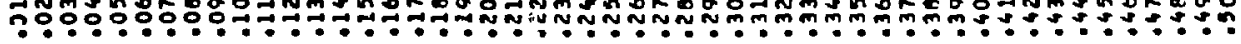




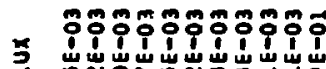

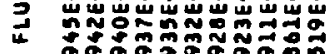

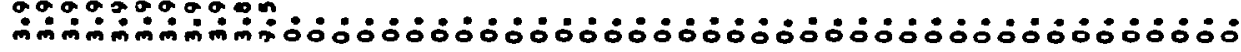

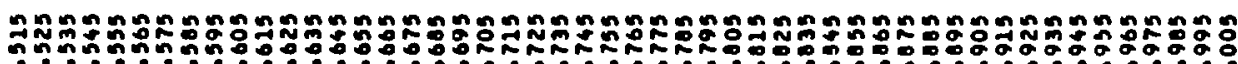

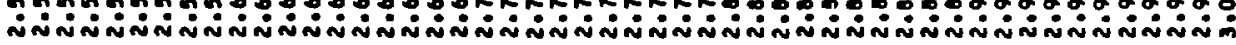

\section{క}

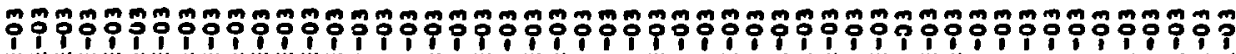

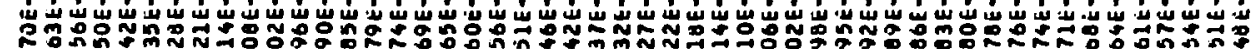

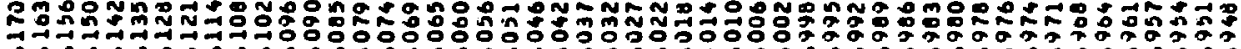

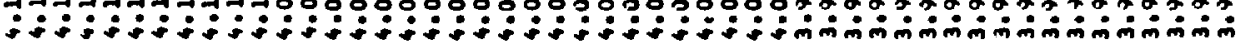

훈

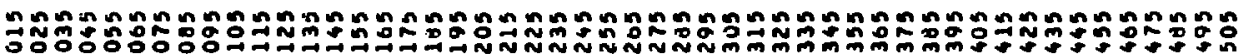

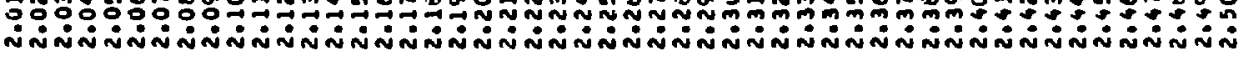

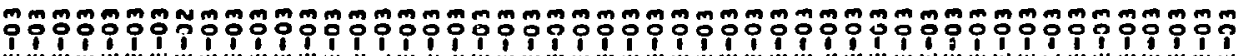

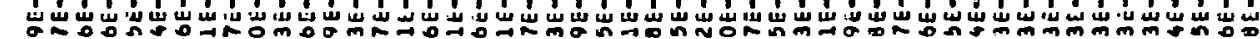

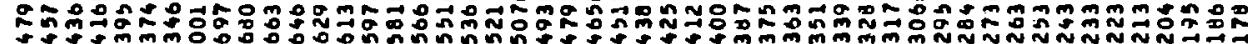

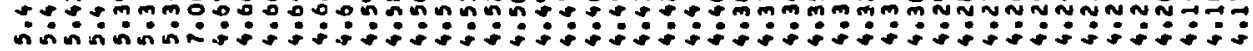

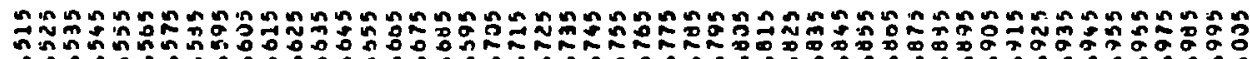

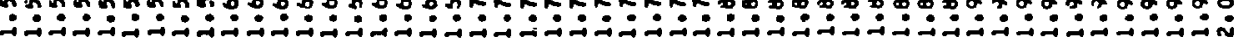
n mmmmmmmmmmmmmmmmmmmmmmmmmmmmmmmmmmmmmmmmm

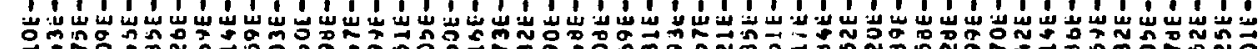

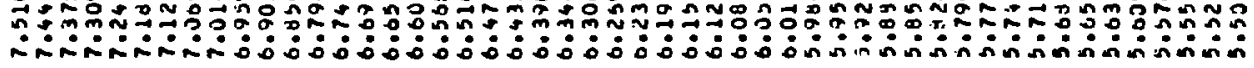

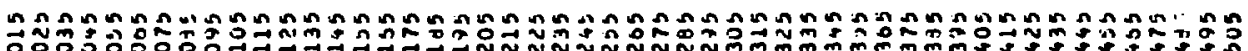
过

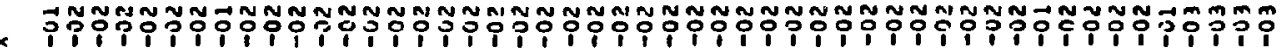

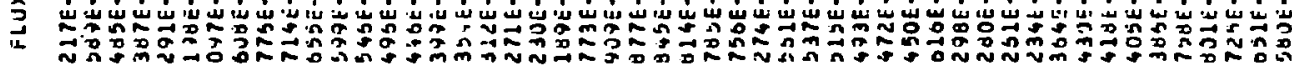

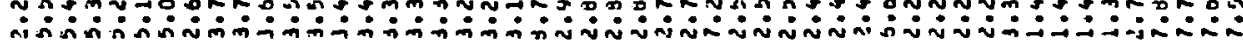

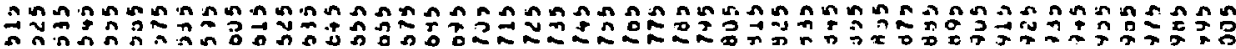

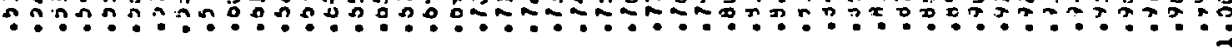

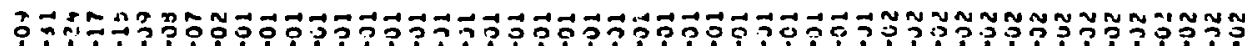

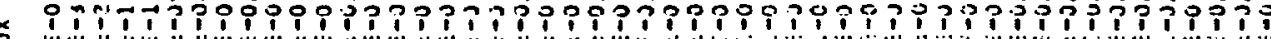

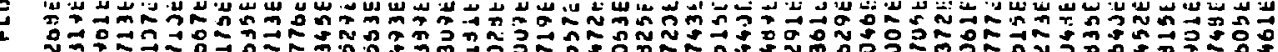

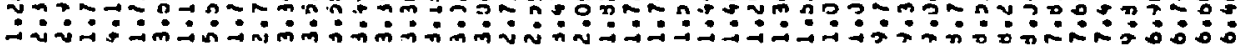

离

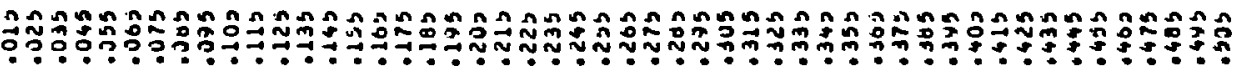




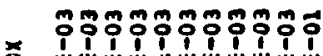

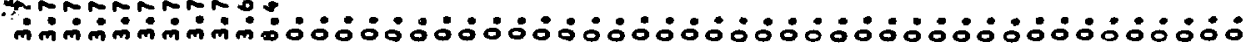

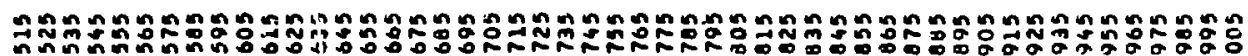

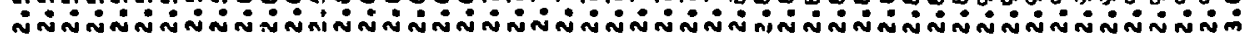

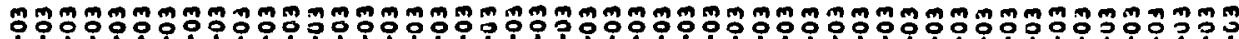

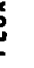

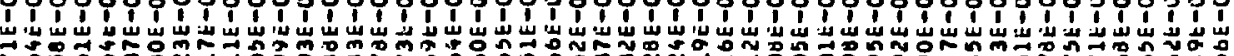

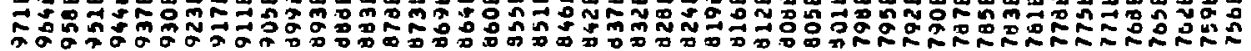

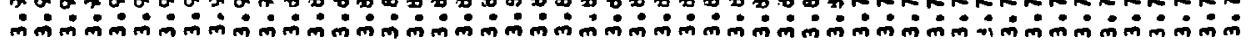

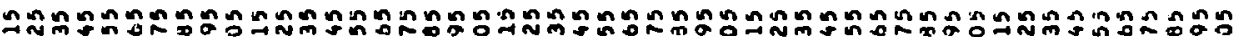

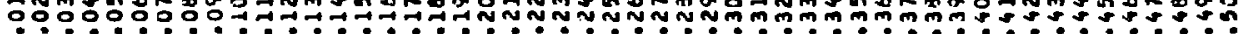

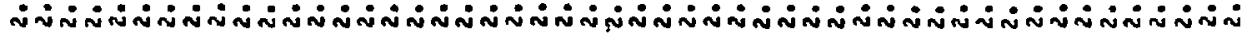

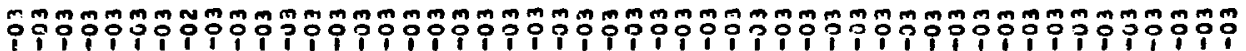

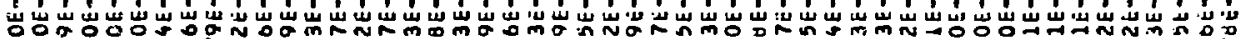

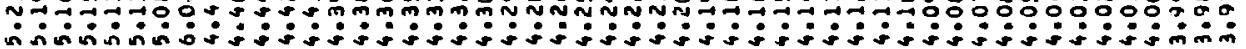

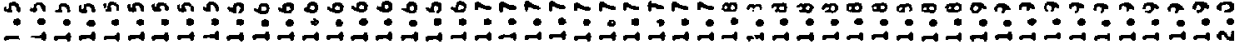

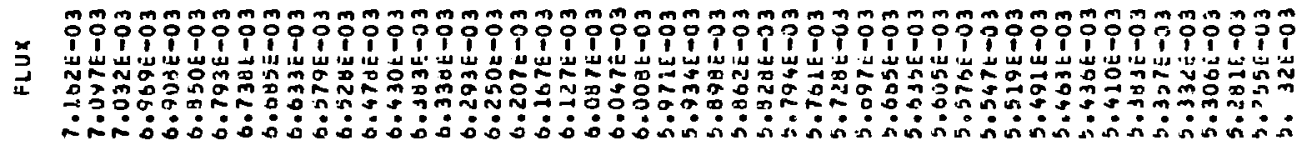

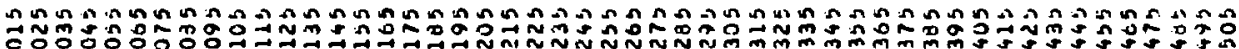

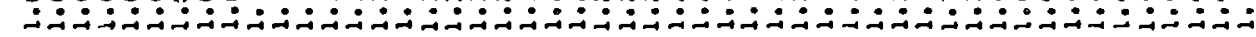

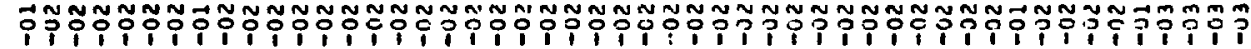

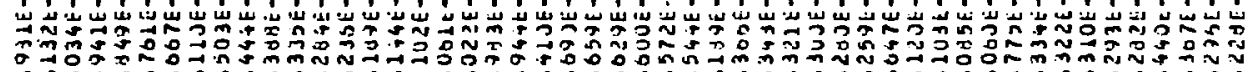

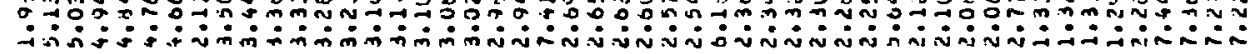

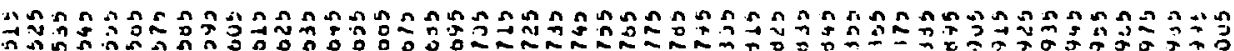

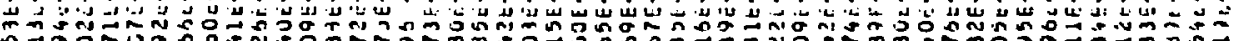

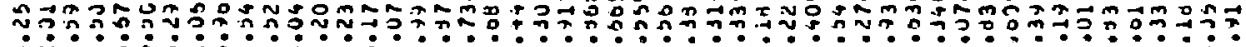

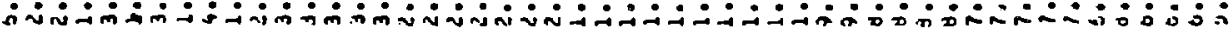




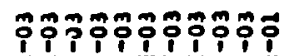

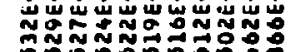

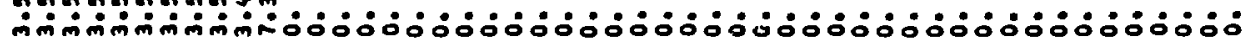

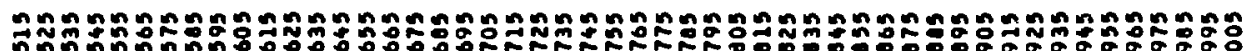

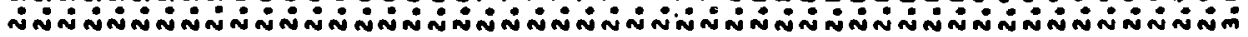

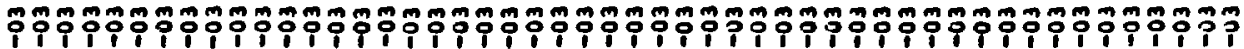
ílilid

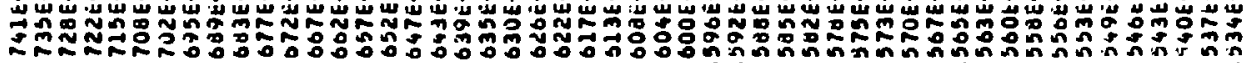

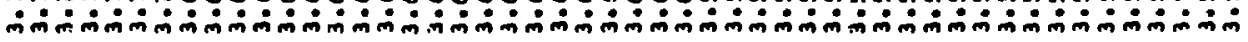

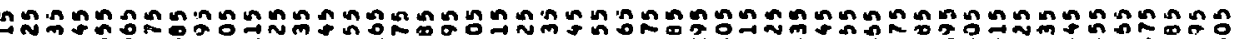

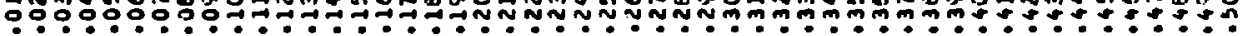

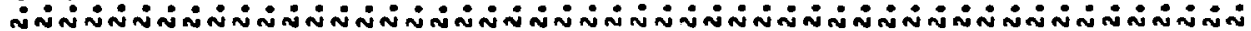

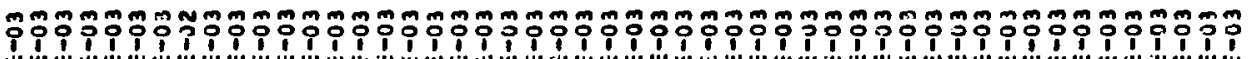

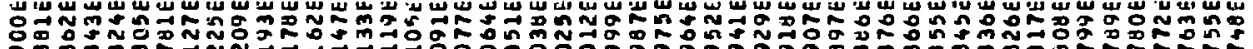

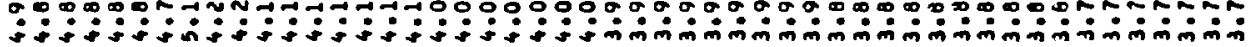

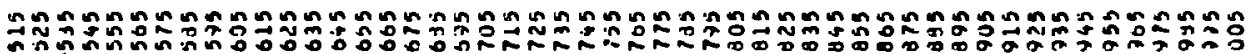

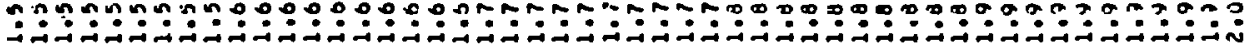

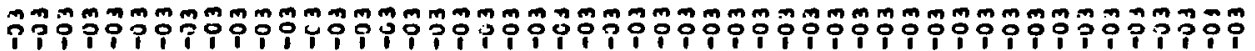

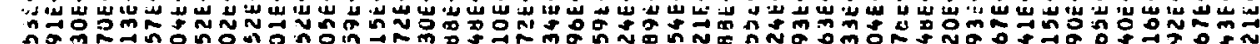

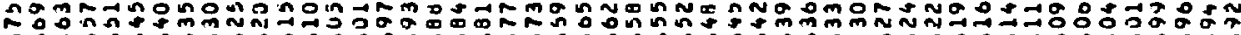

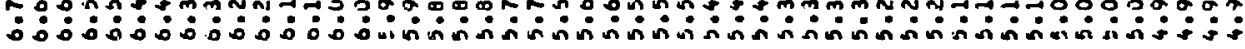

$\zeta$

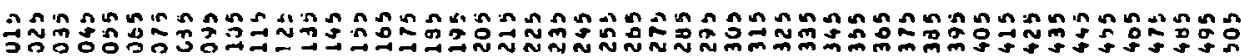
过

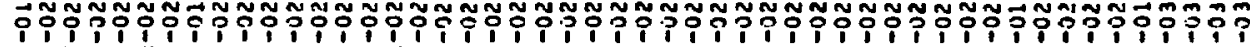

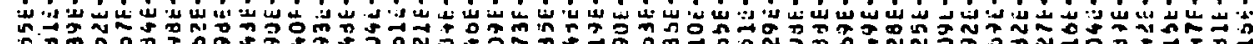

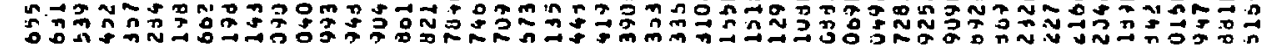

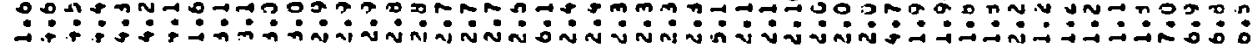

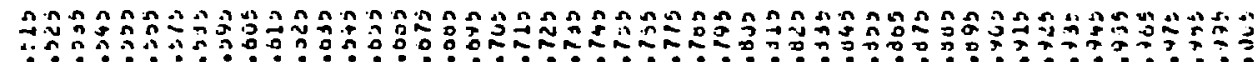

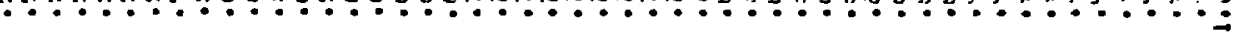

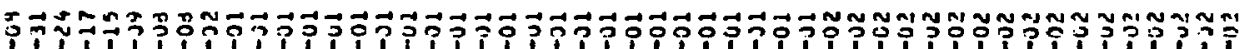

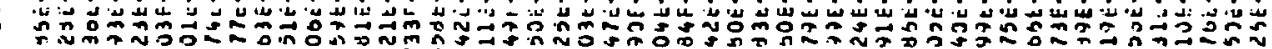

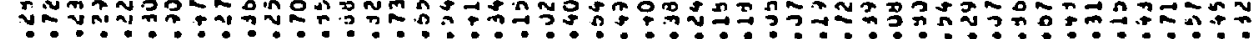

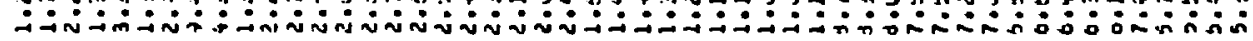




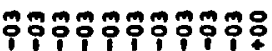

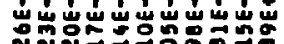

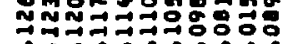

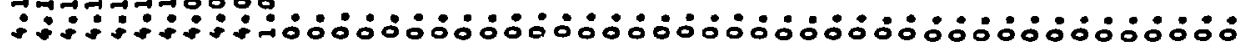

¿े

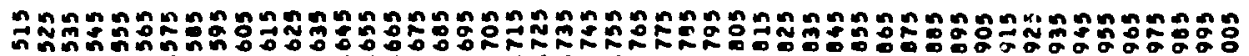

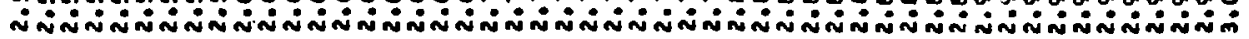

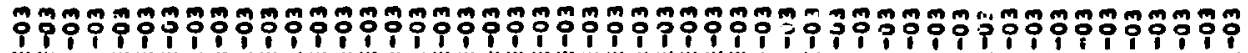

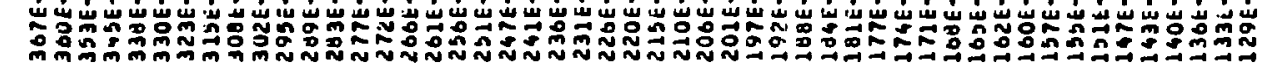

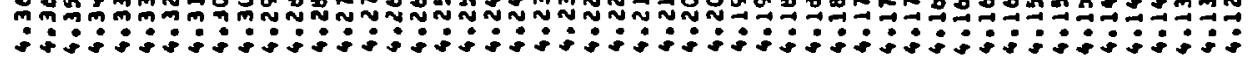

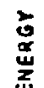

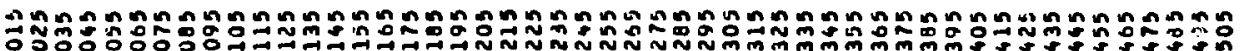

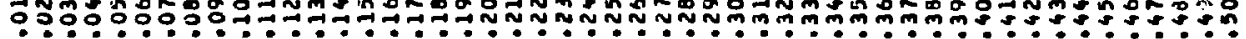

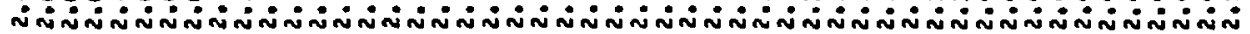

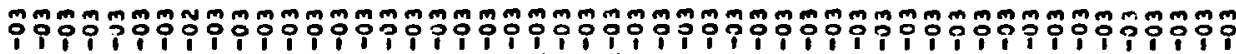
山س山س

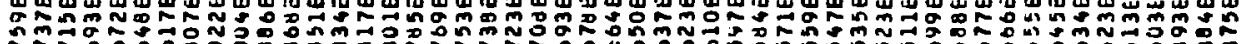

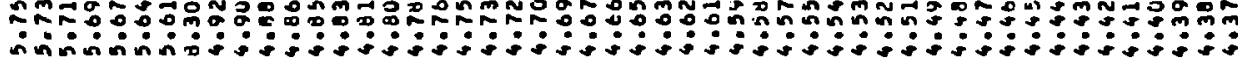

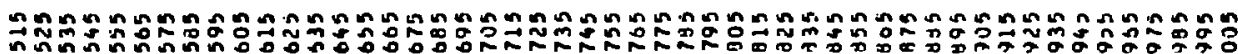

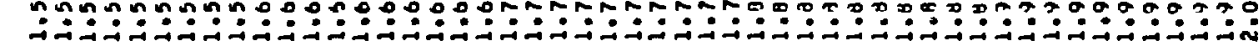

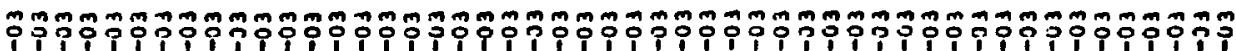

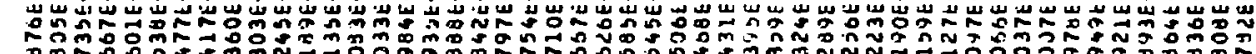

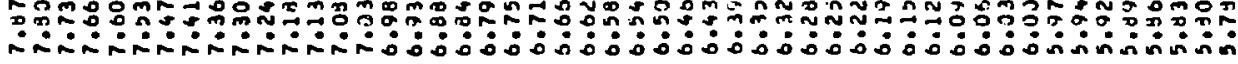

2.

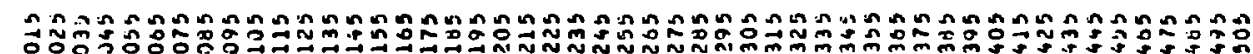

乙

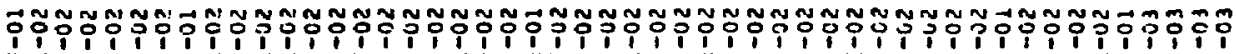

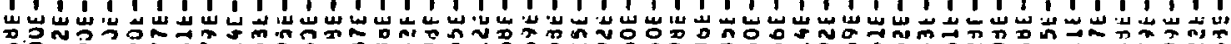

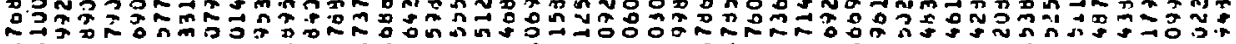

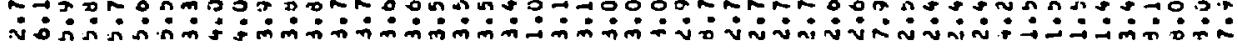

$\rightarrow$

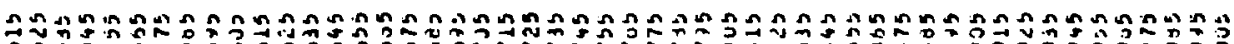

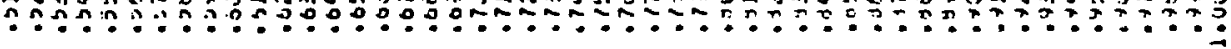

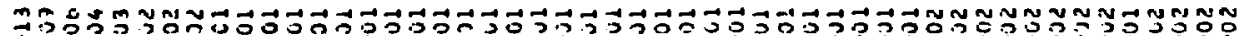

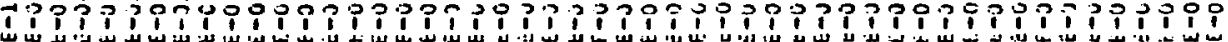

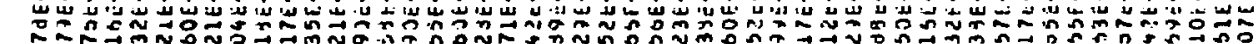

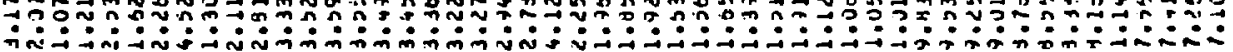

न 


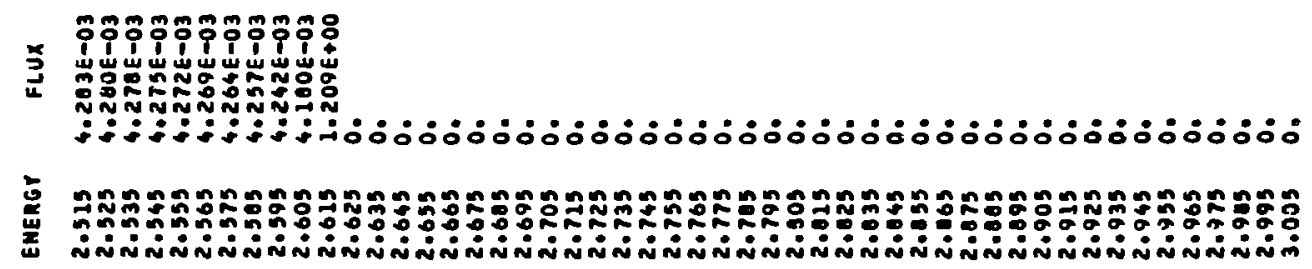

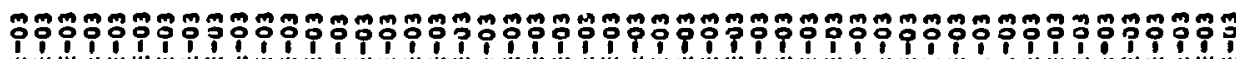

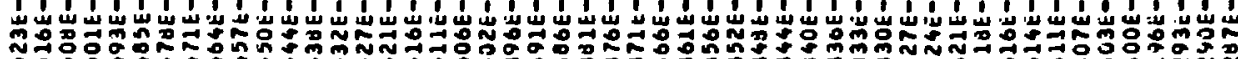

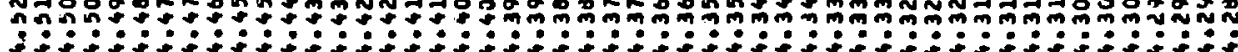

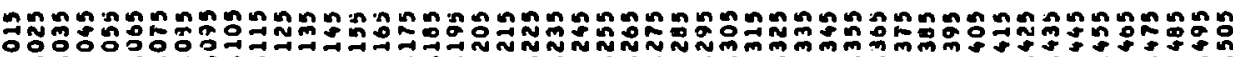

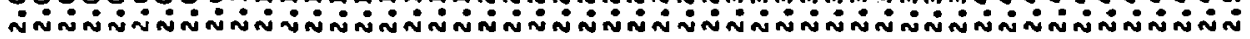

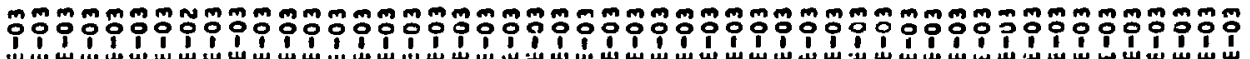

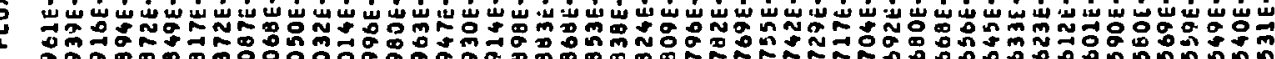

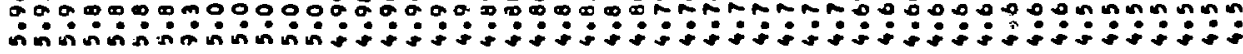

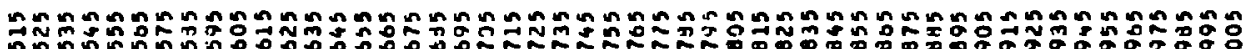

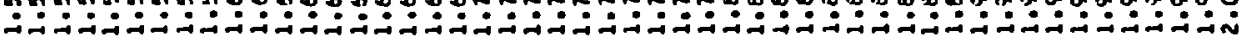

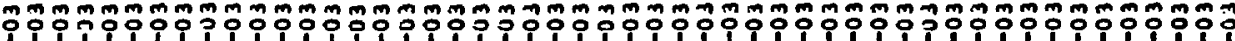

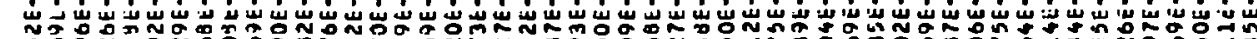

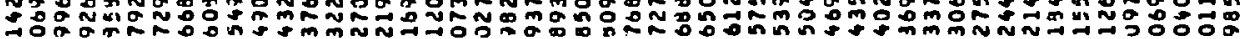

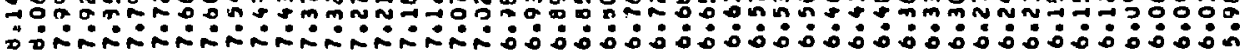

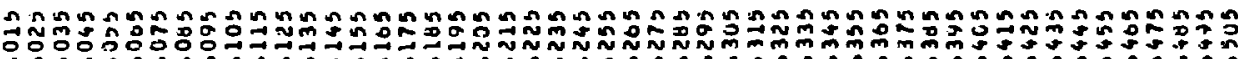

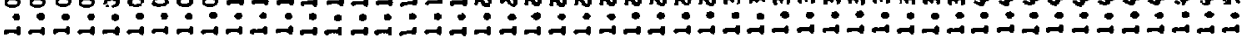

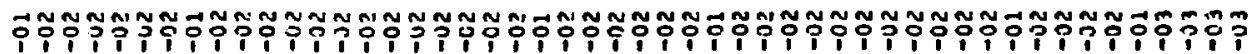

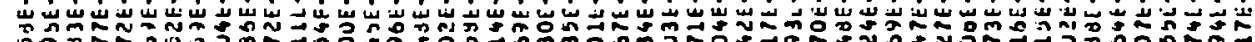

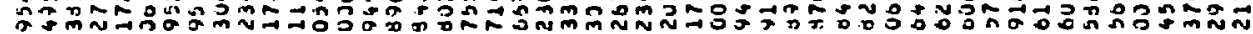

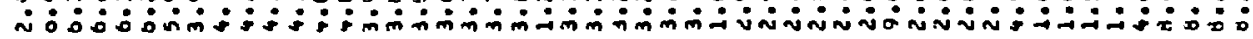

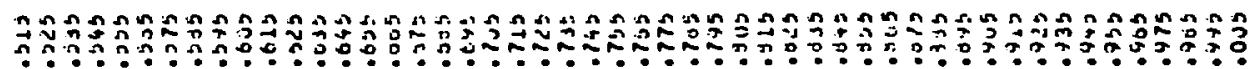

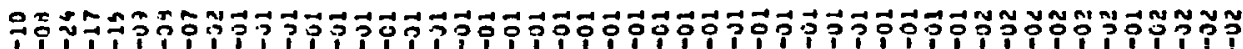

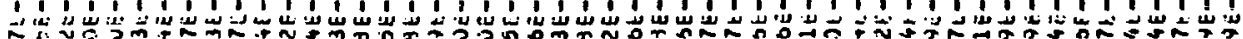

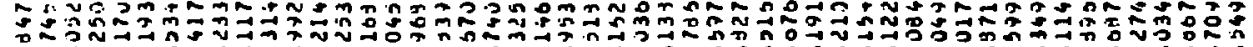

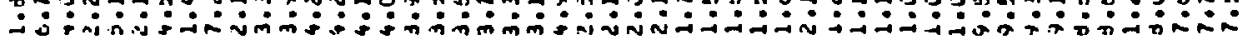




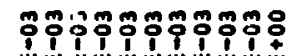

3

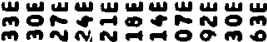

N N N N N N

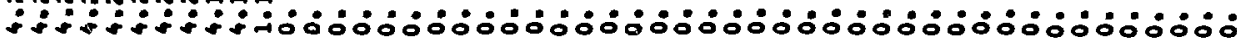

总

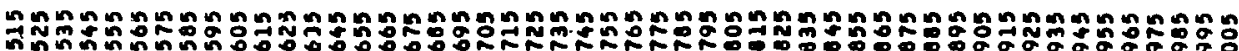

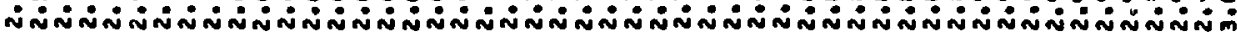

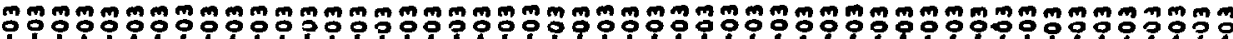

i i i

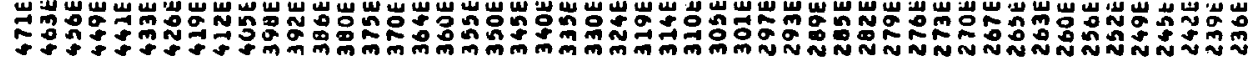

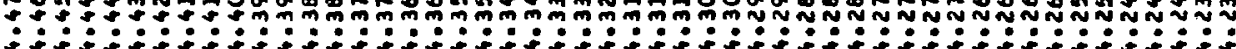

宸

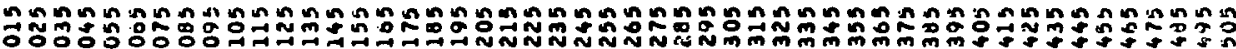

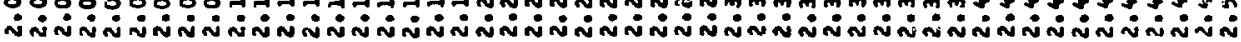

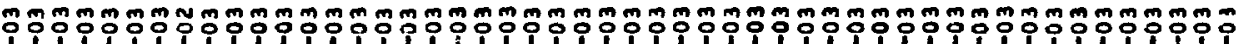

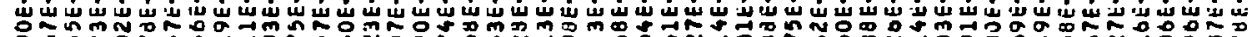

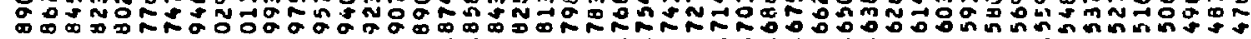

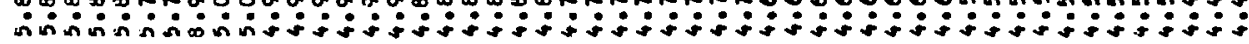

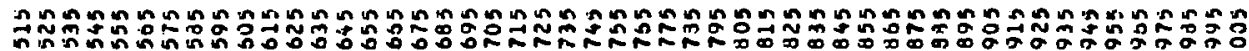

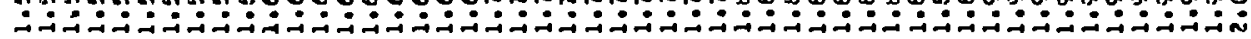

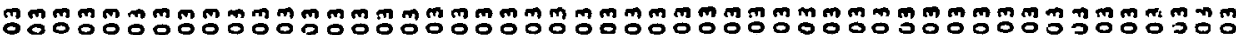

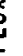
管

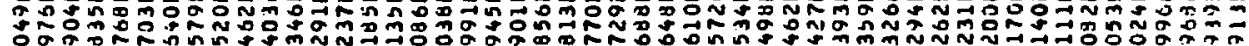

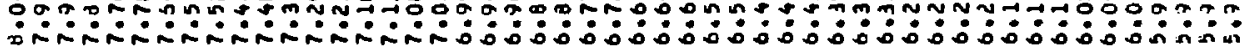

$\vec{x}$

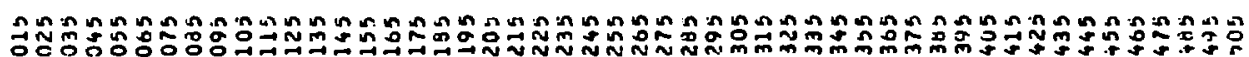

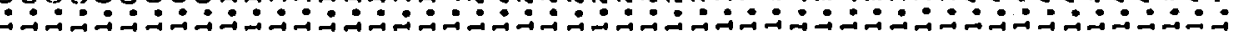

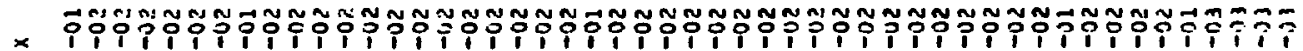

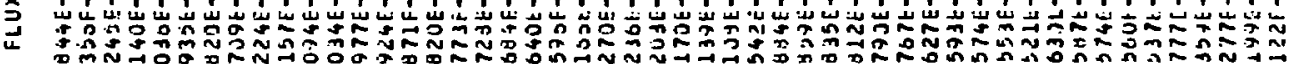

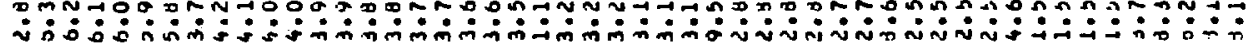

$>$

7. 要

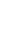

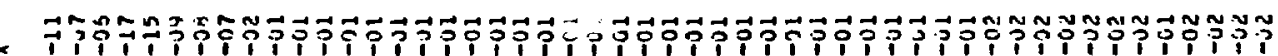

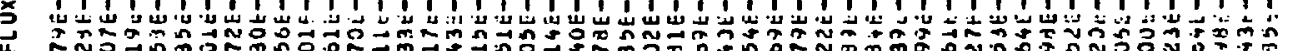

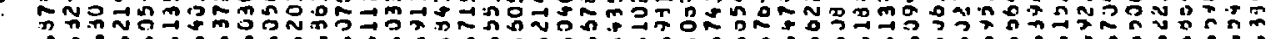

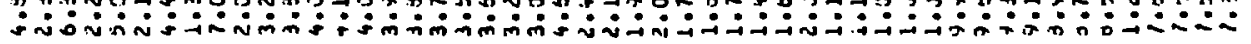

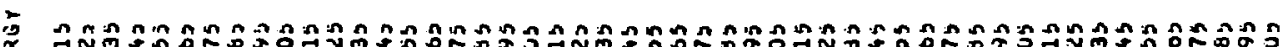

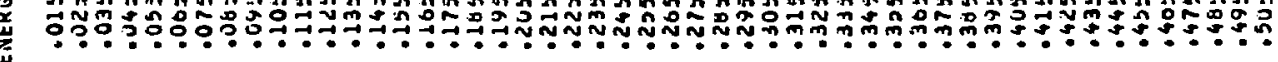


$\stackrel{x}{\mathbf{u}}$

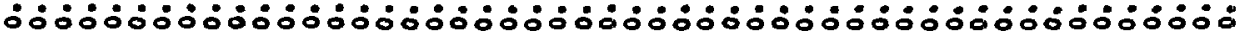

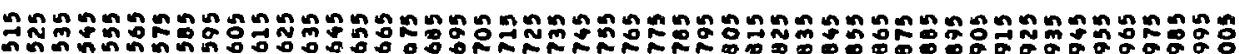

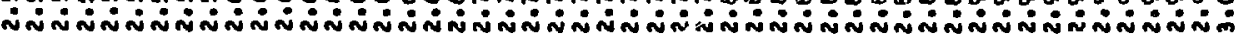

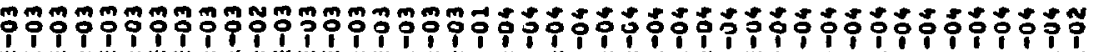

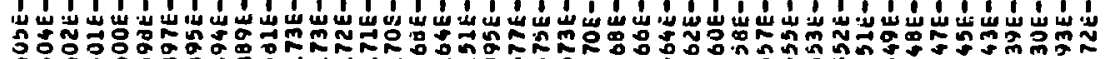

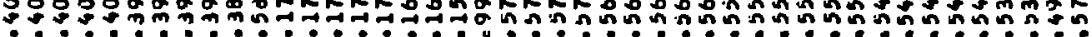

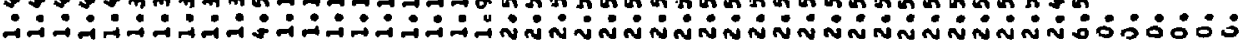

7๓

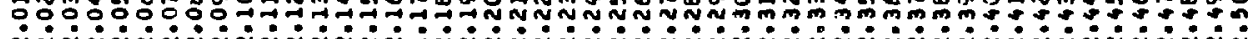

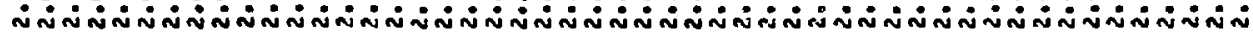

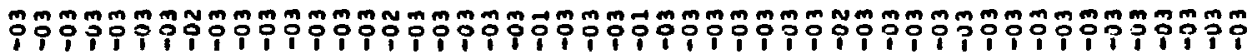

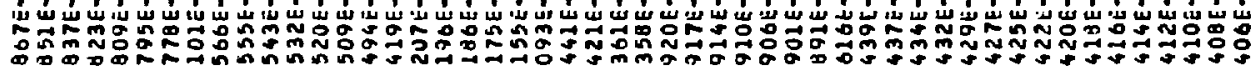
on

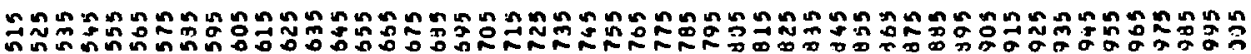

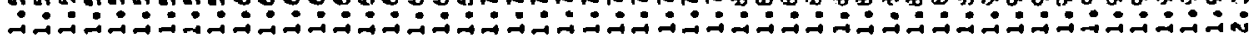

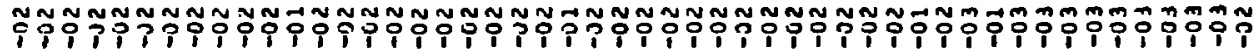

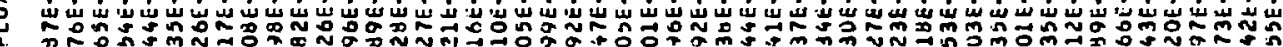

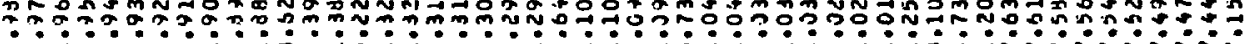

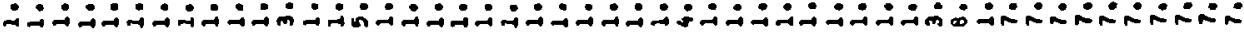

ל

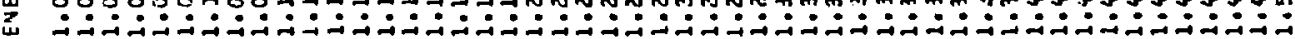

$\stackrel{2}{2}$

$\therefore \stackrel{x}{2}$

2

它

$\rightarrow$

3

5

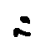

$\hat{\sim}$

岁

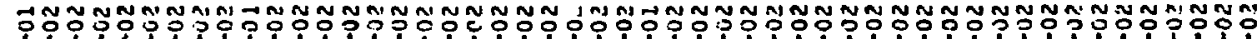

I

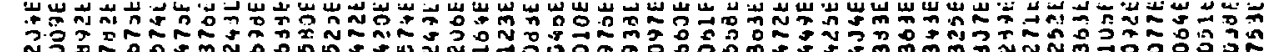

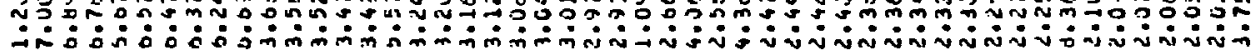

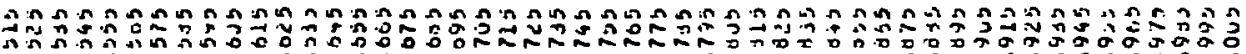

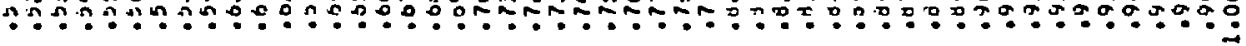

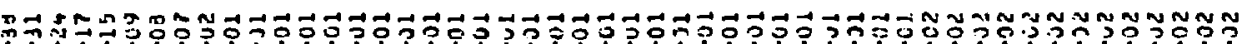

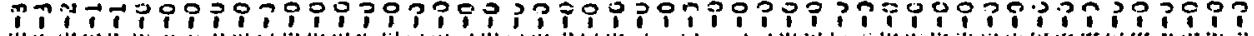

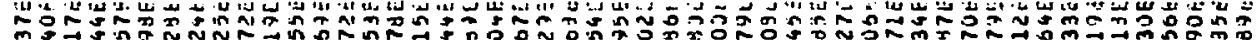

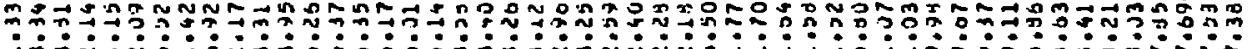

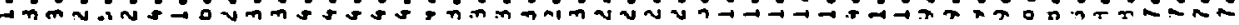

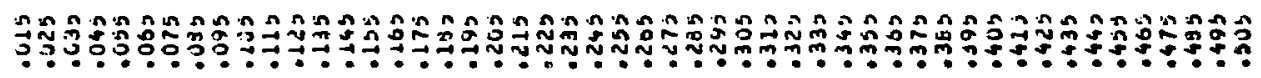


$\stackrel{x}{3}$

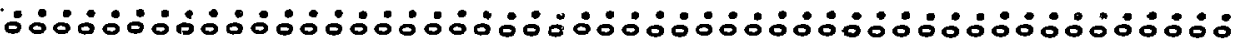

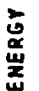

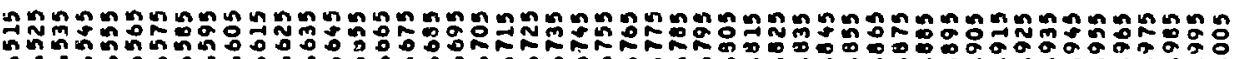

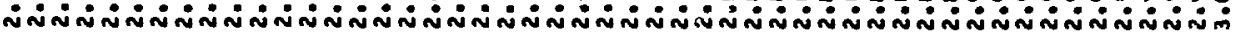

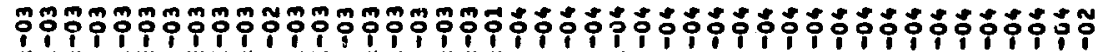

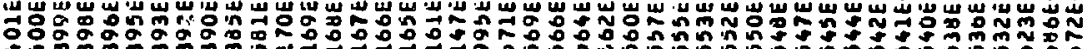
† nem

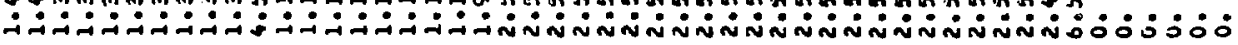

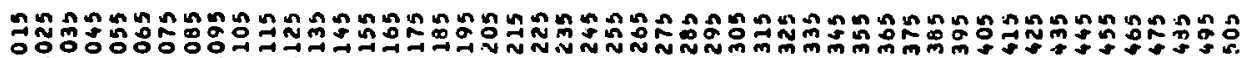

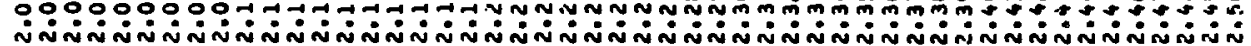

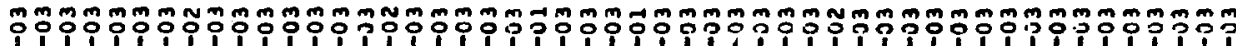

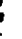

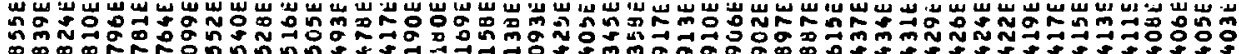

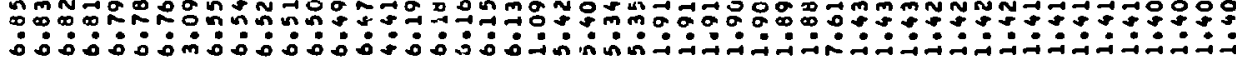

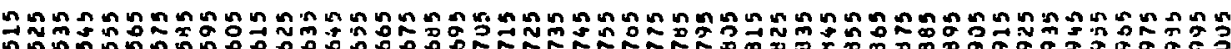

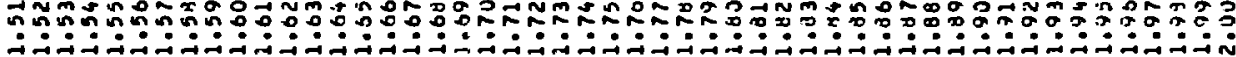

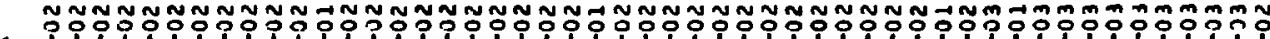

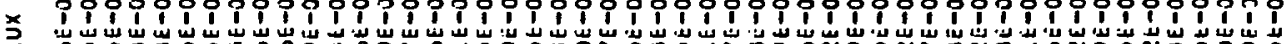

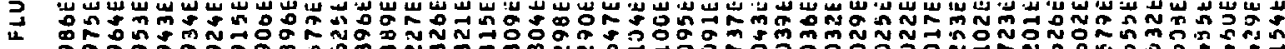

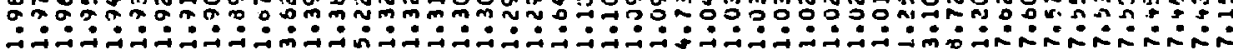

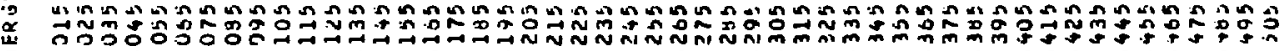

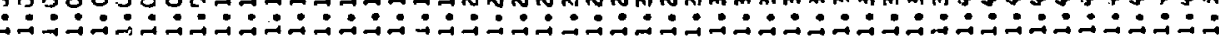

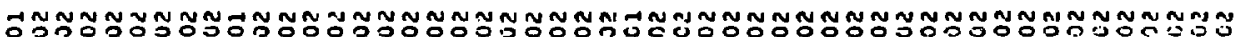
PPTPPip

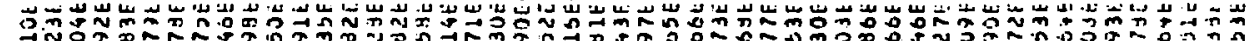

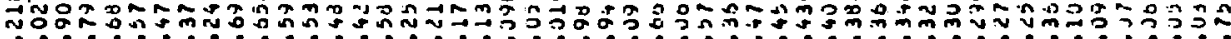

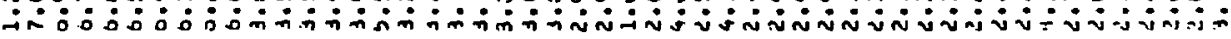

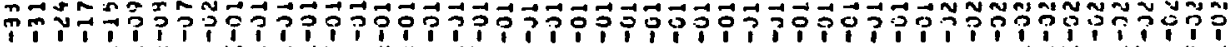

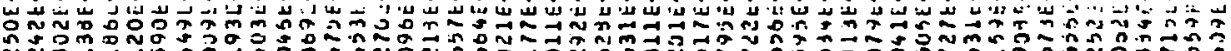

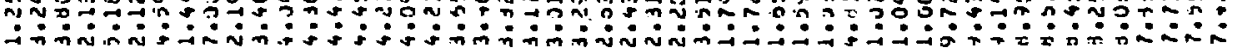


$\stackrel{3}{3}$

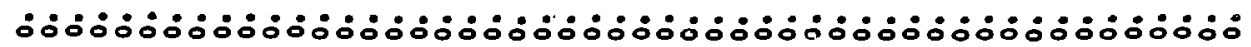

这

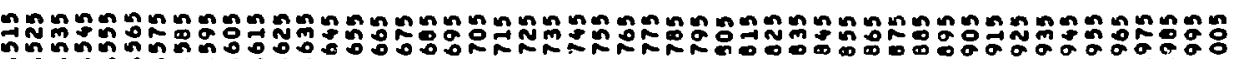

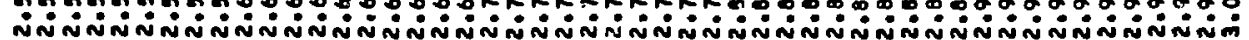

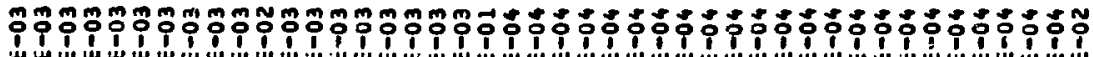

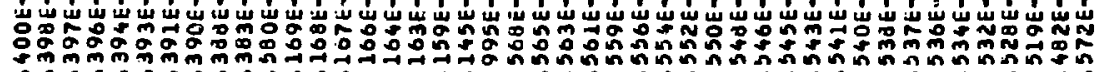

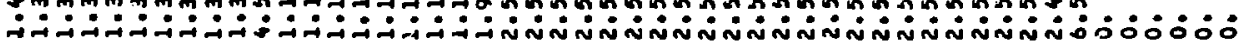

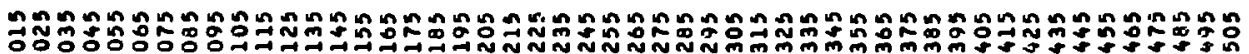

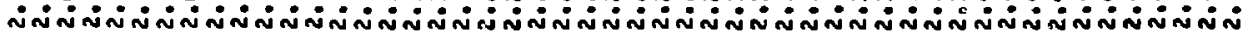

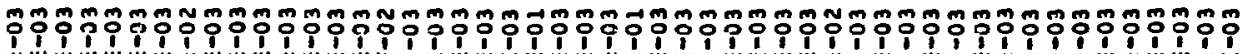

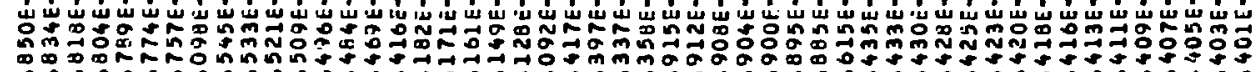
:

N N N N N N N N

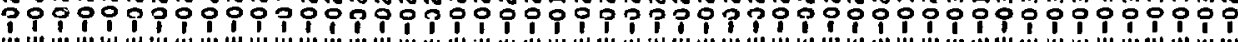

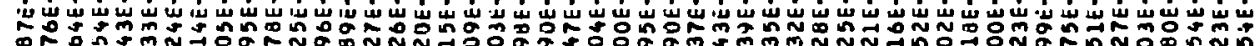

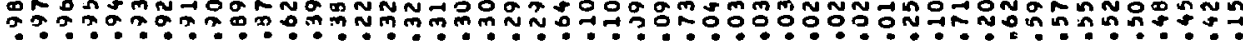

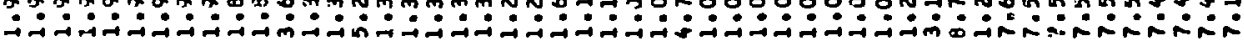

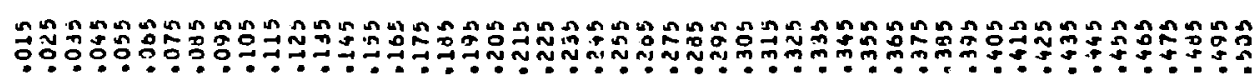

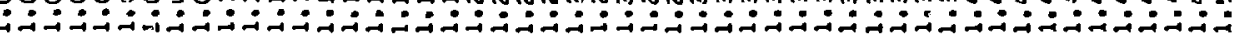

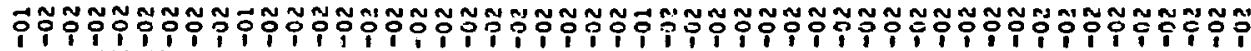

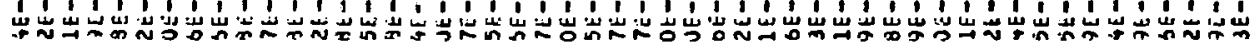

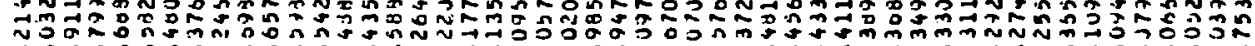

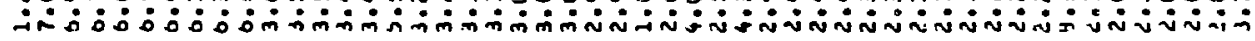

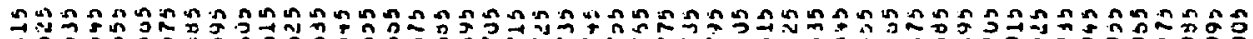

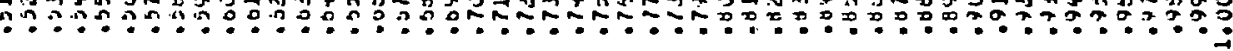

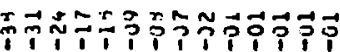

$\vec{p} \vec{i} \vec{i} \vec{i} \hat{i}$ 


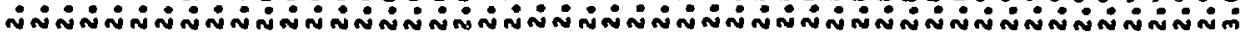

\section{$\stackrel{3}{3}$}

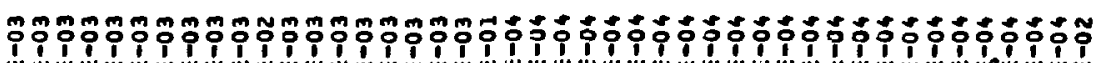

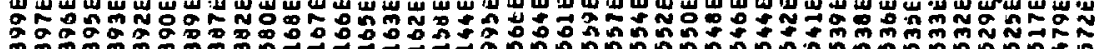

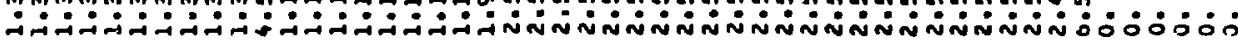

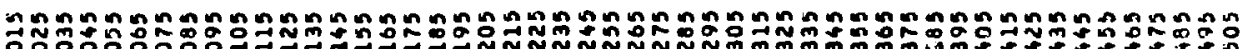

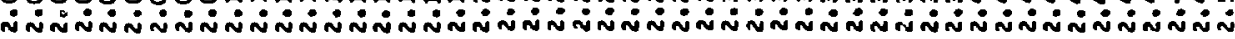

m

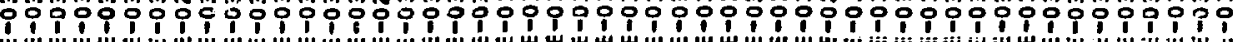

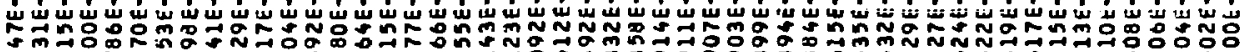

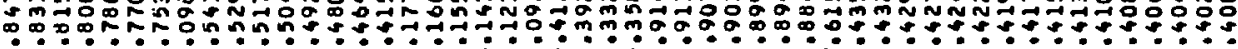

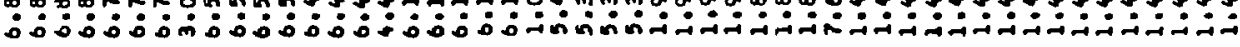

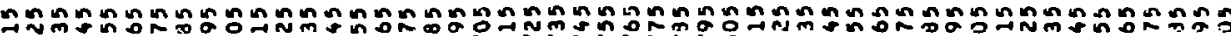

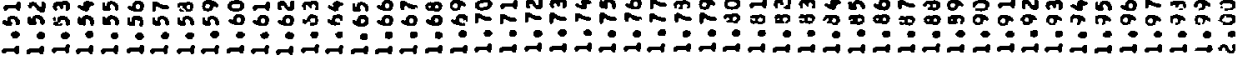

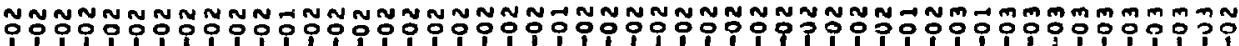

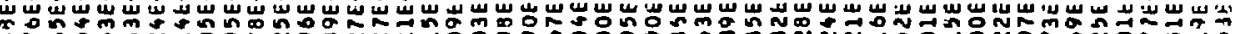

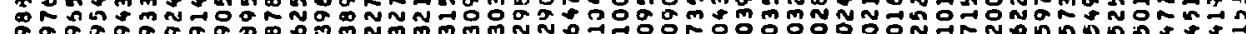

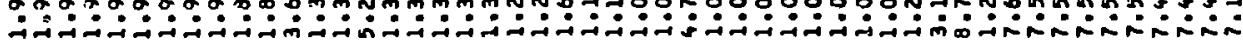

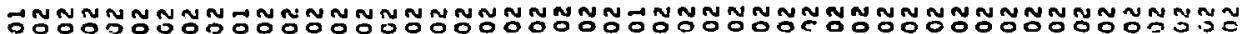

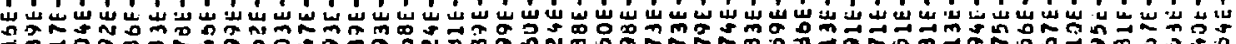

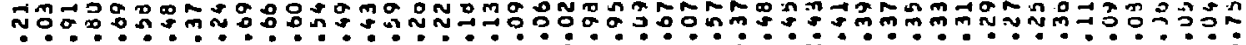

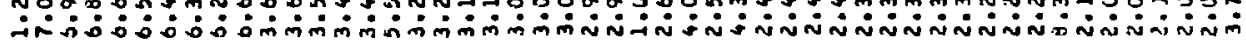

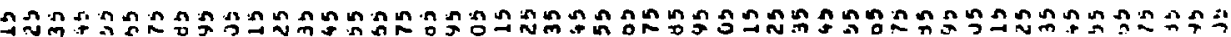

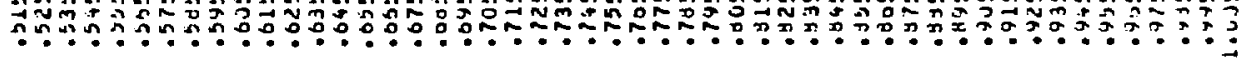

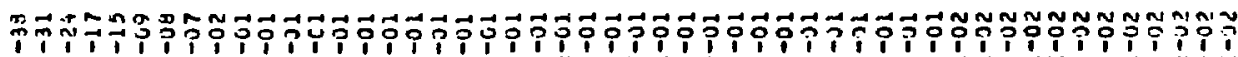

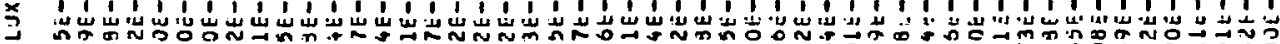

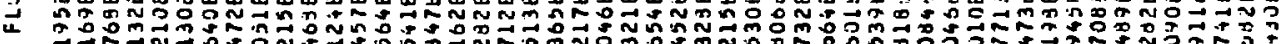

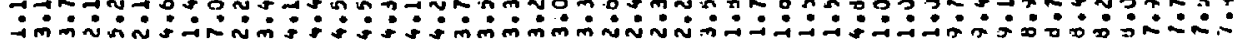


喜

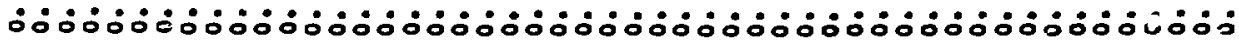

产

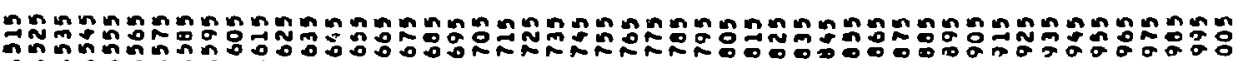

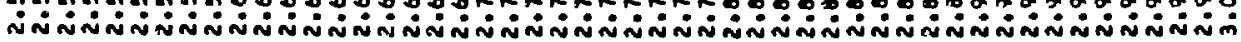

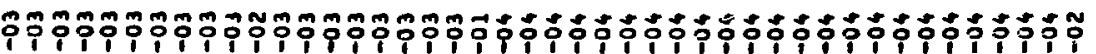

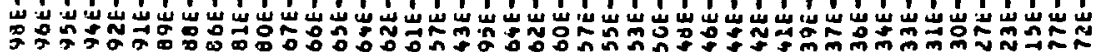

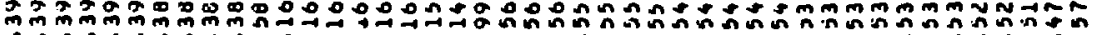

:

3

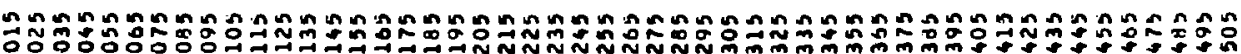

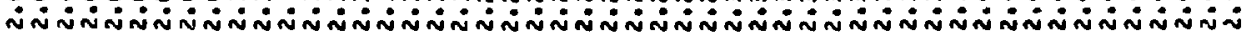

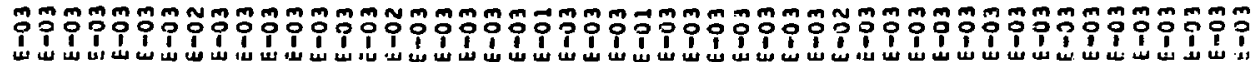

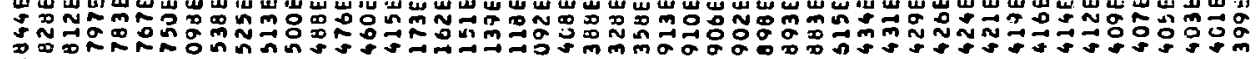
:

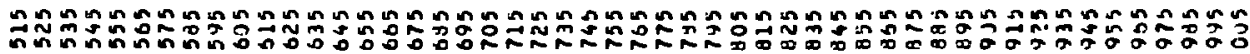

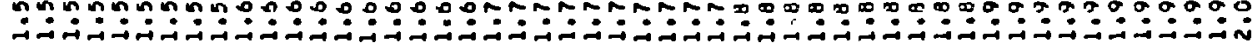

$\stackrel{\square}{3}$

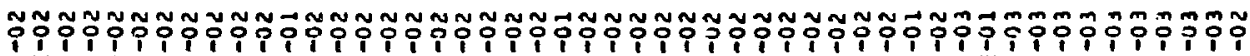

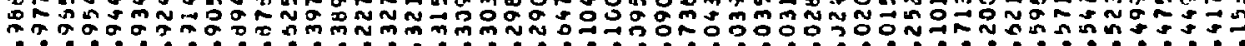

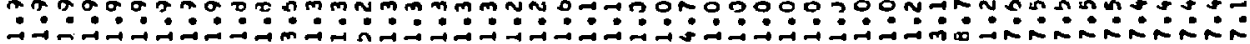

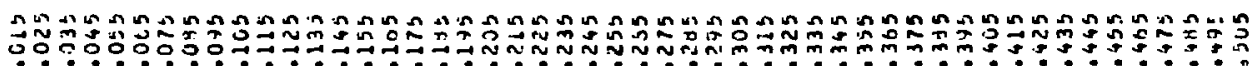

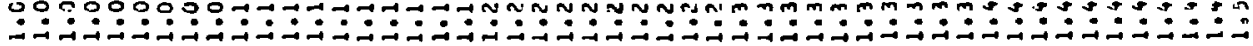

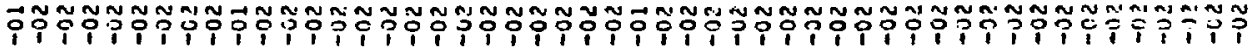

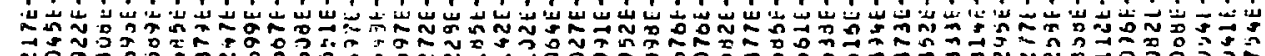

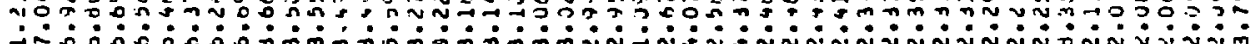

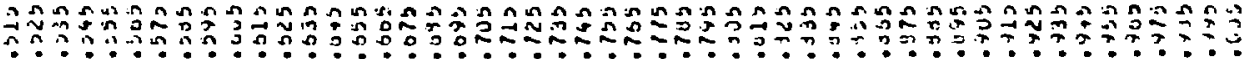

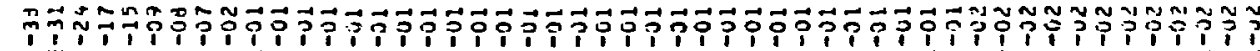

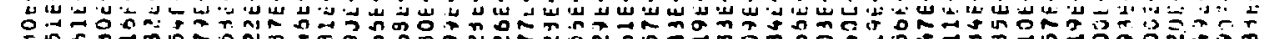
-

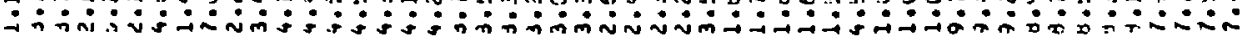

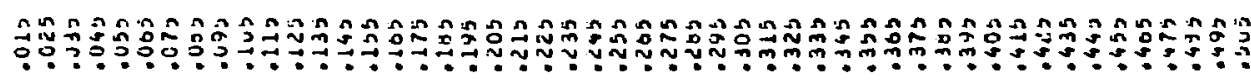




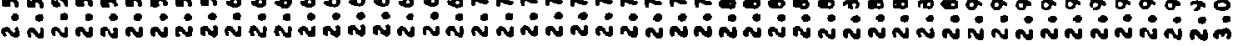

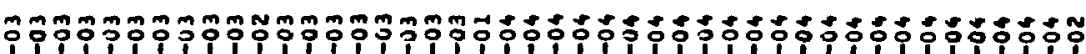

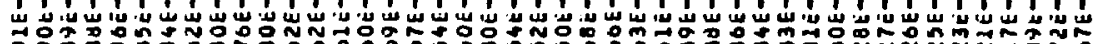

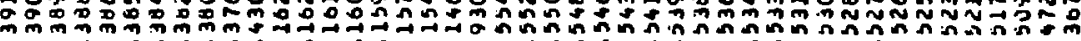

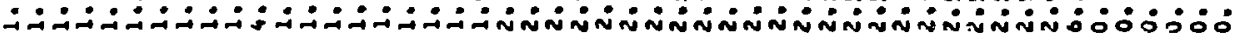

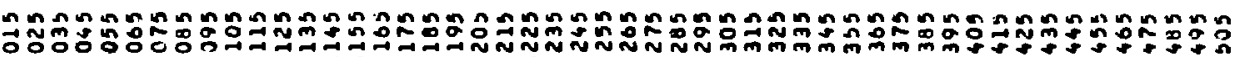

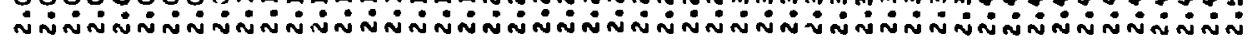

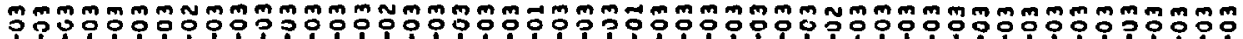

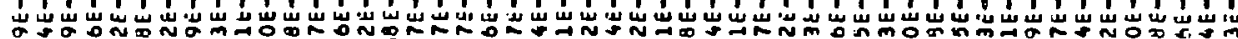

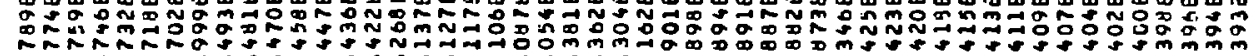

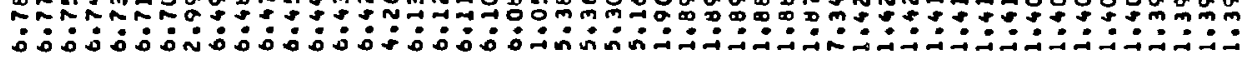

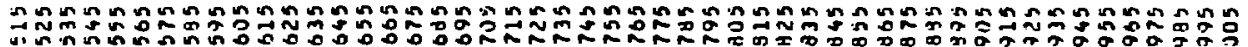
บ:

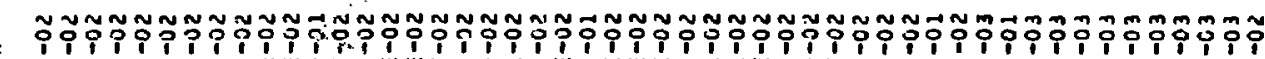

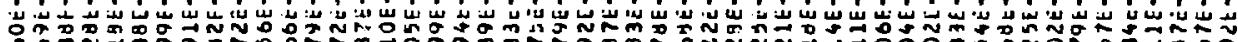
然.

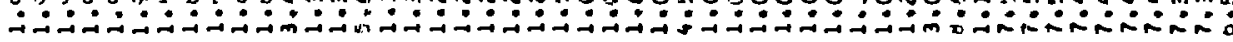

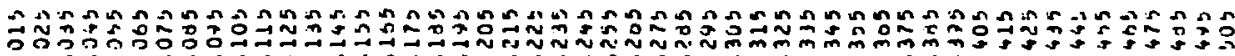
ت里

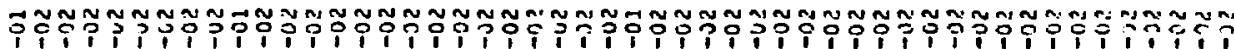

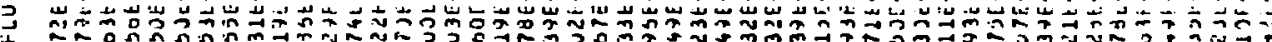

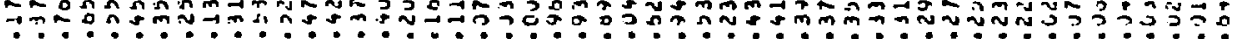

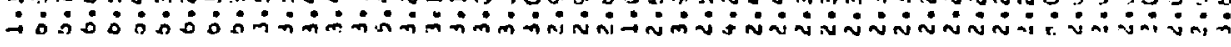

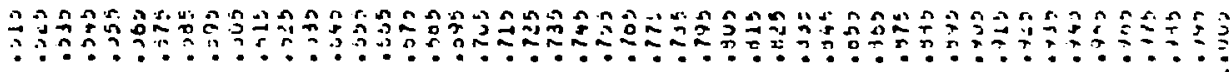

;

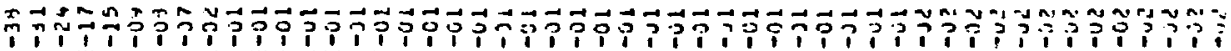

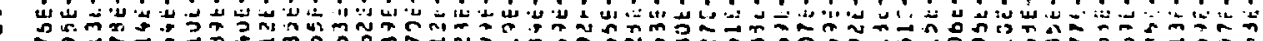

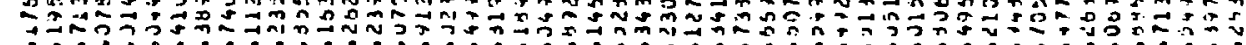

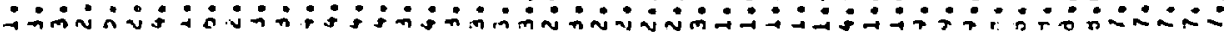

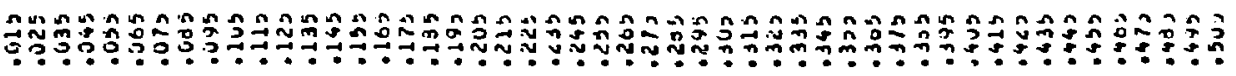




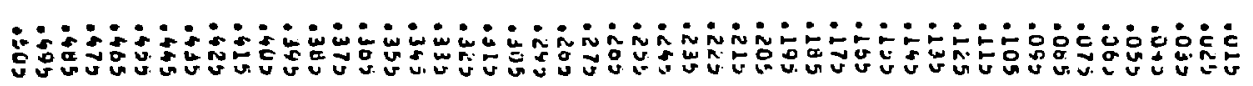

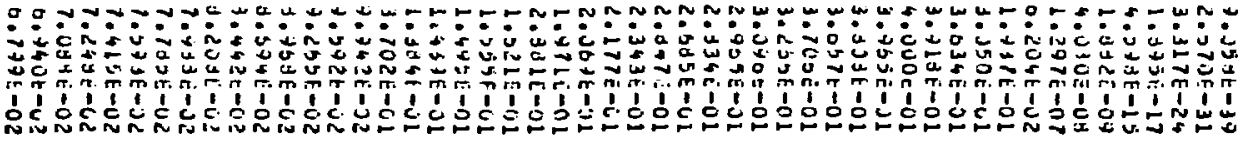

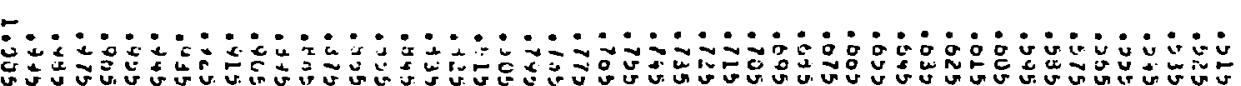
momomomomomons

* :

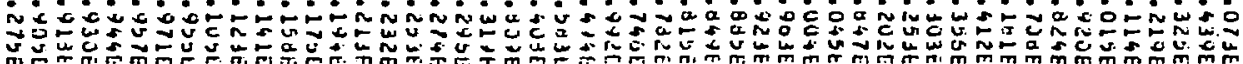

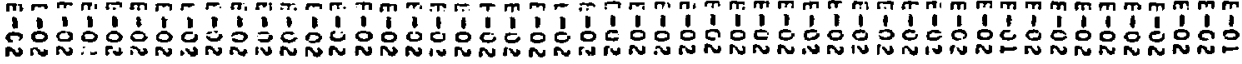

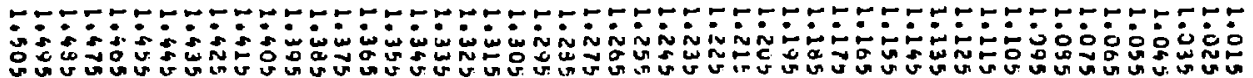

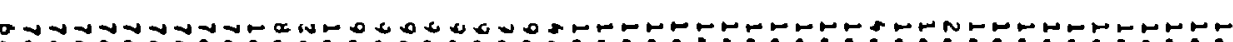

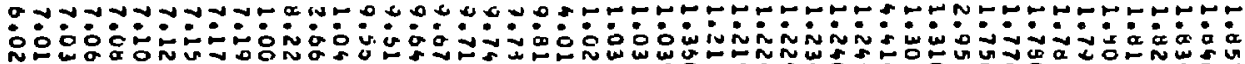

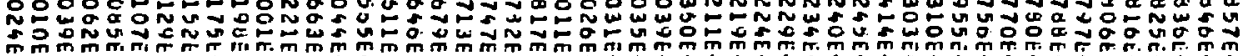

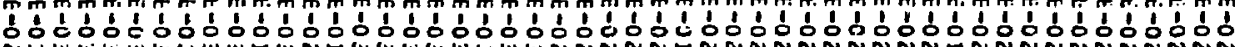

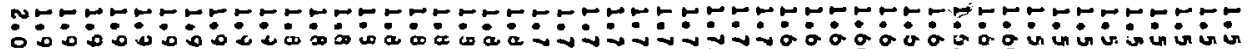

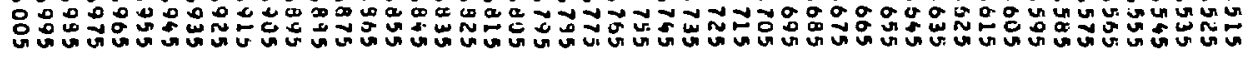

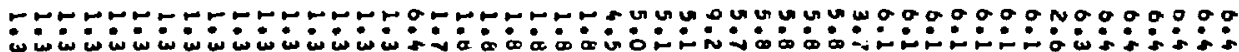

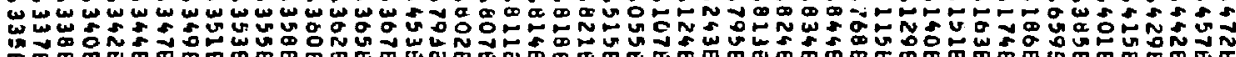

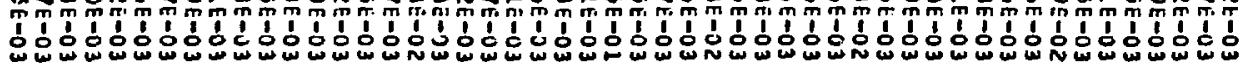

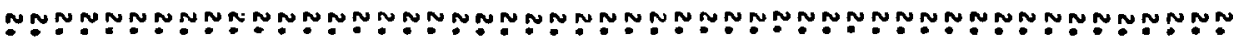

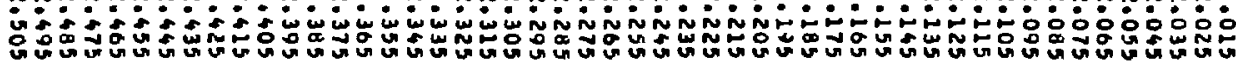

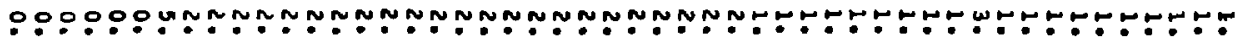

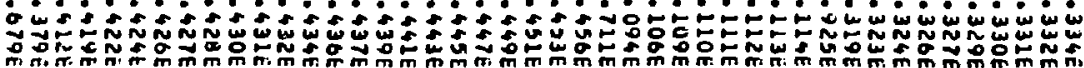
b.joj

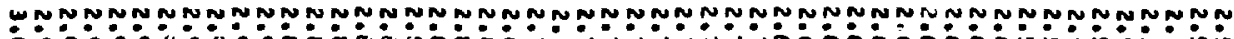

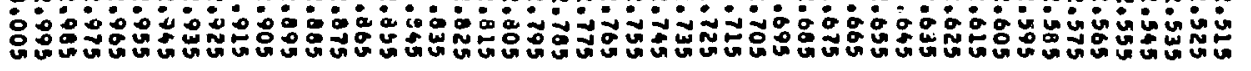


蛋.

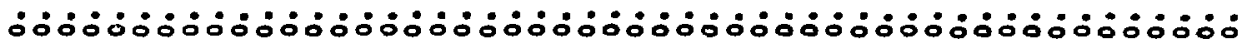

立

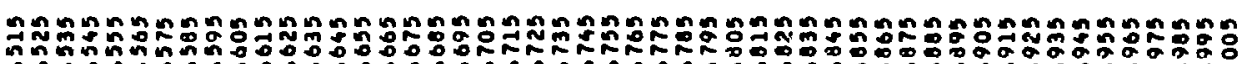

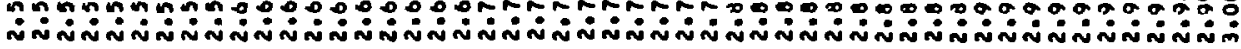

\section{崫}

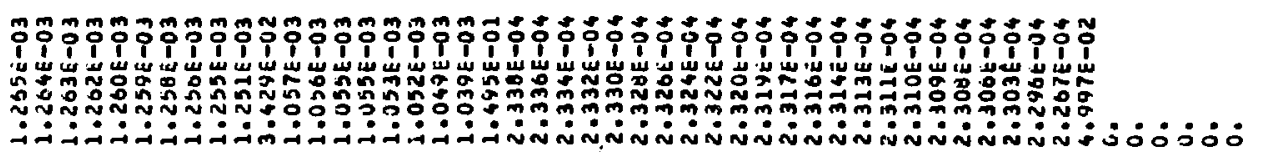

立

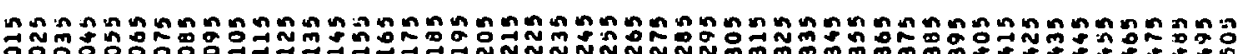

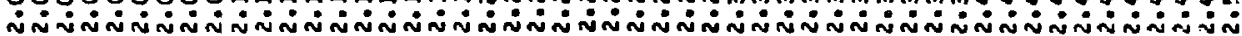

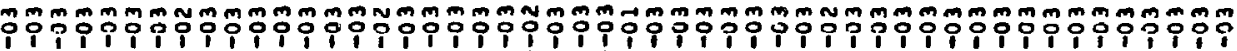

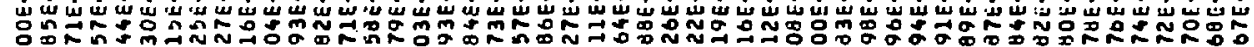

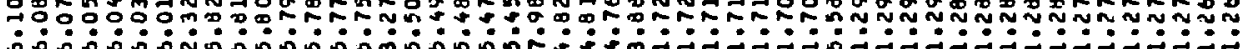

n

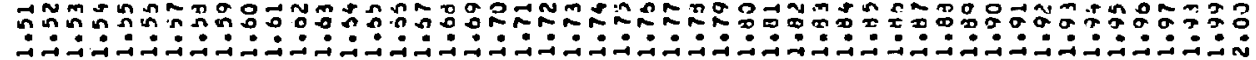

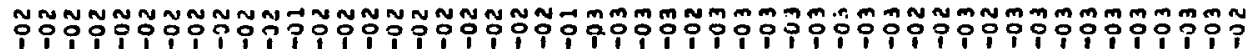

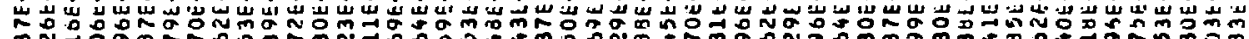
N N

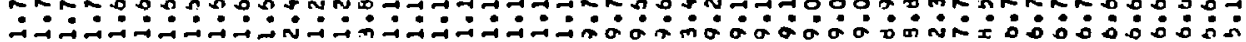

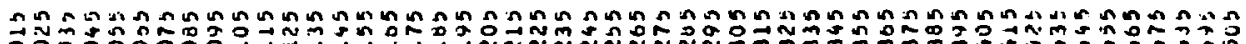

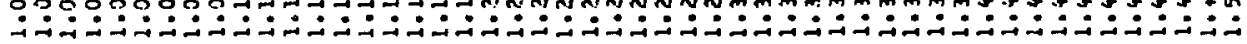

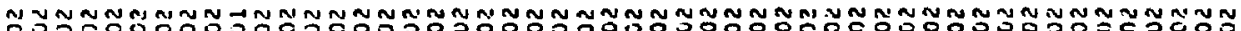

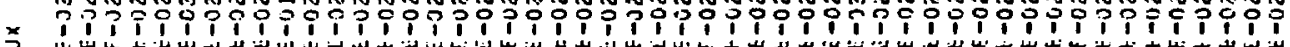

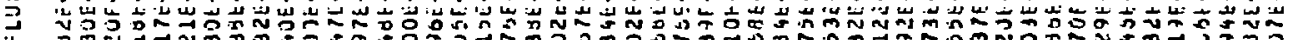

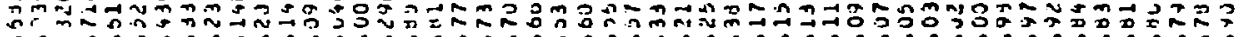

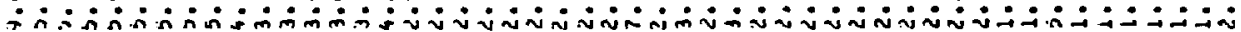

(1)

6)

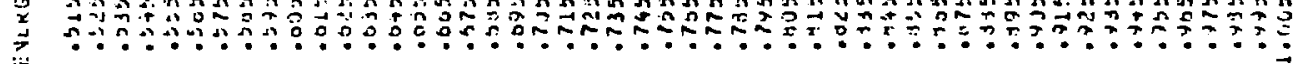

$\div$

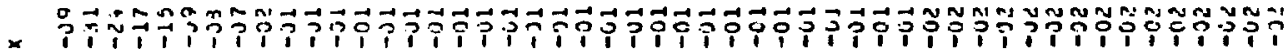

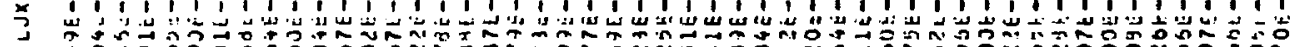

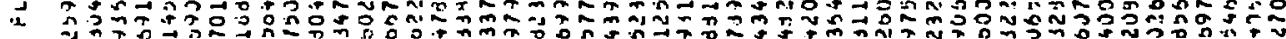

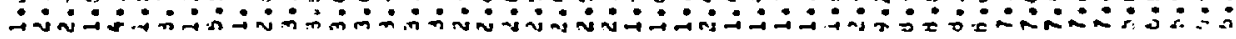

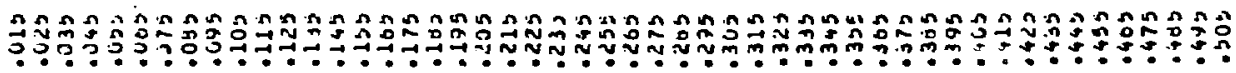




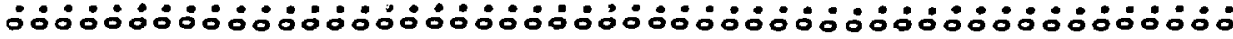

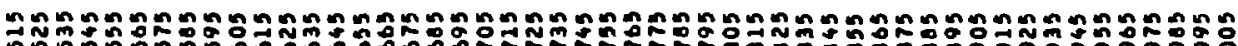

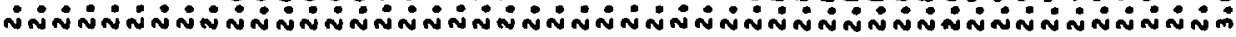

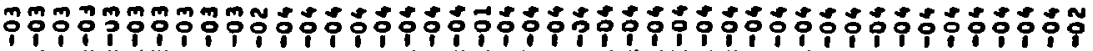

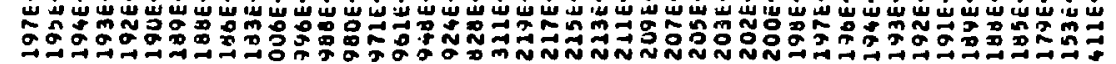

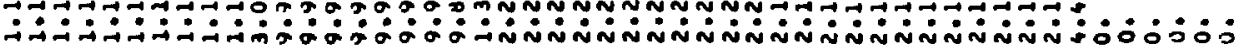

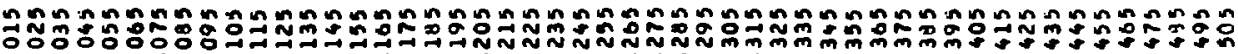

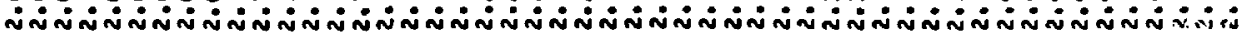

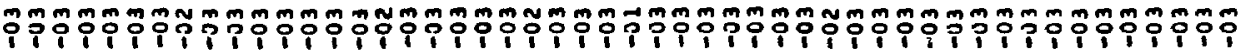

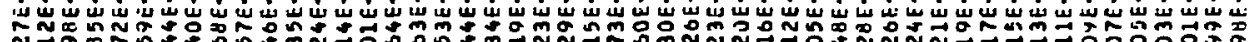

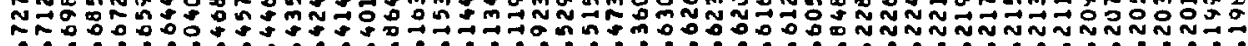

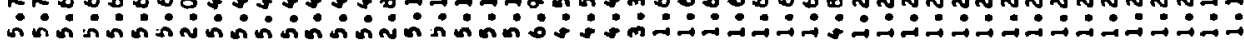

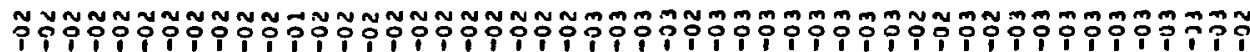

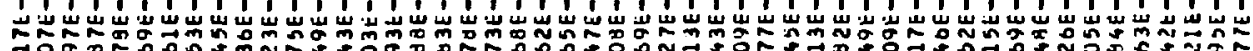

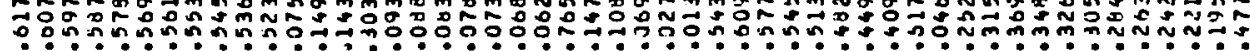

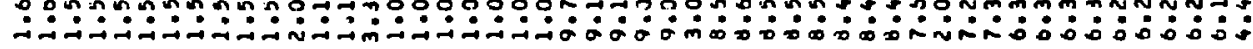

$\stackrel{5}{2}$

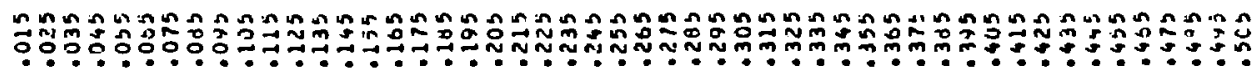

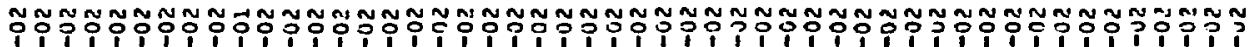

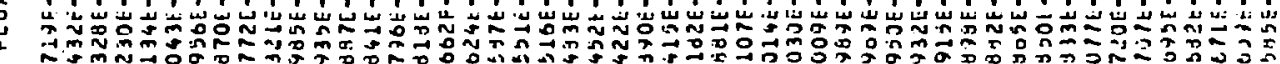

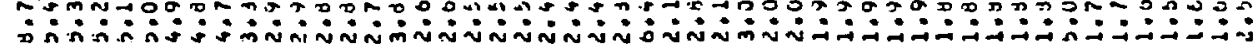

(

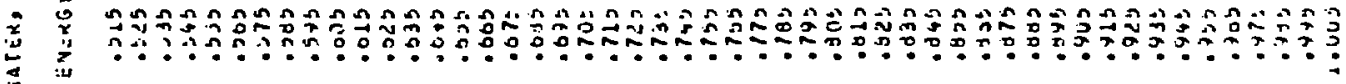

$\div$

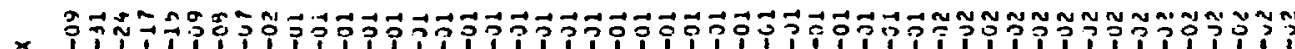

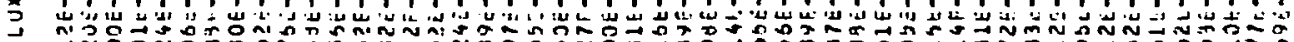

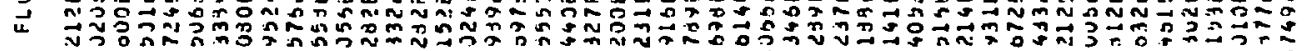

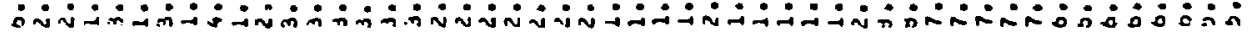

is

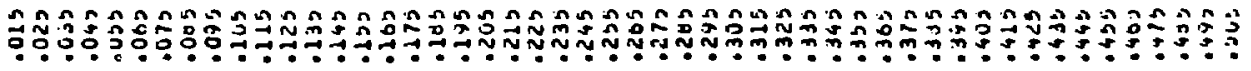




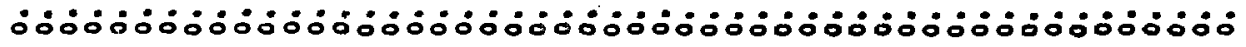

$\stackrel{3}{2}$

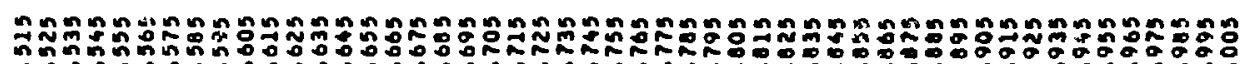

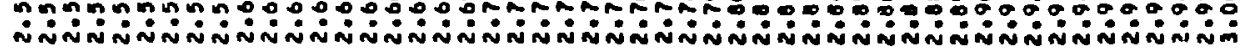

$\stackrel{\mathbf{u}}{\mathbf{u}}$

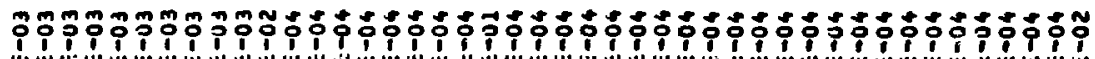

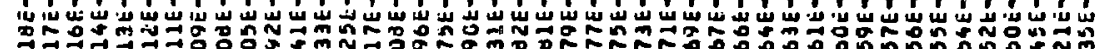

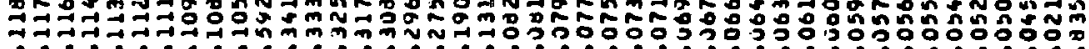

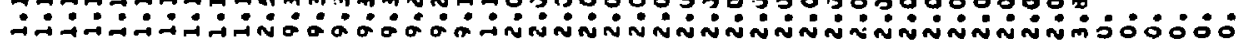

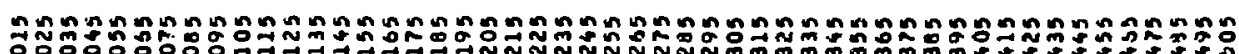

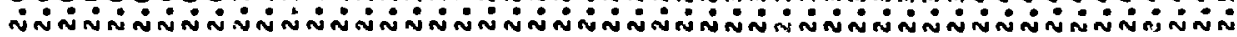

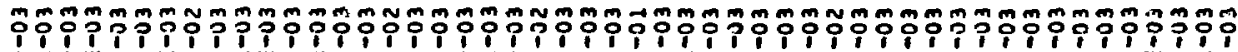

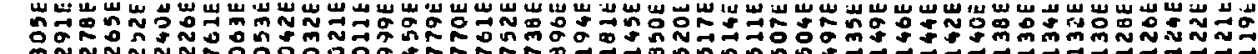

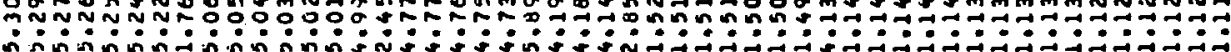

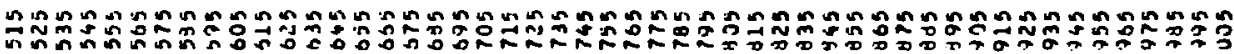

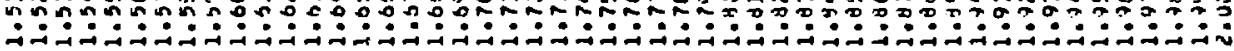

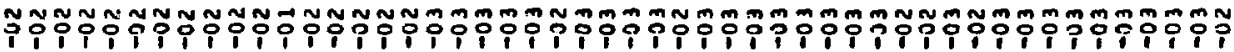

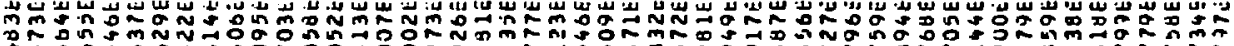

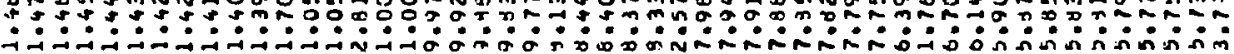

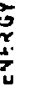

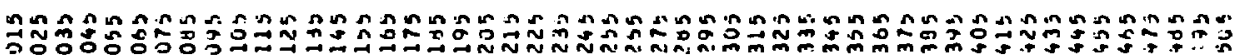

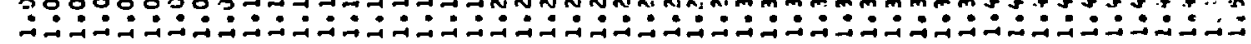

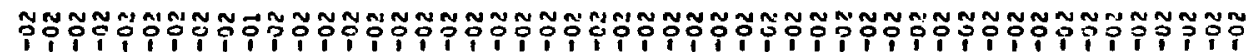

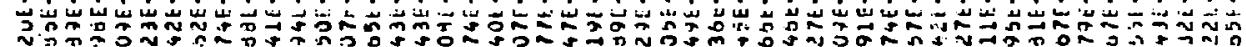

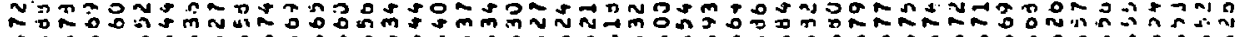

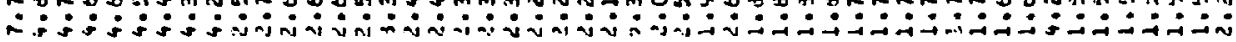

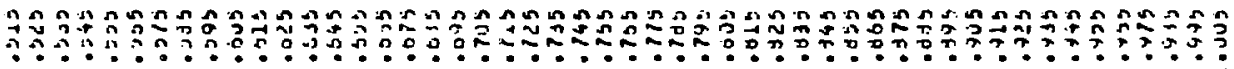

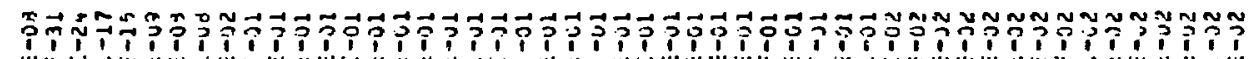

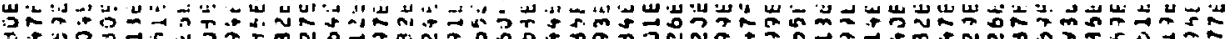

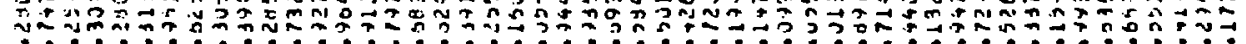

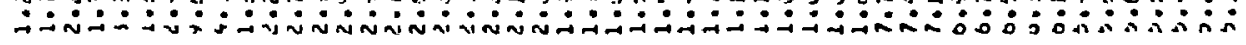




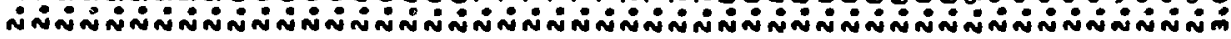

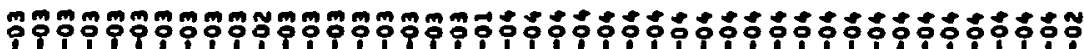

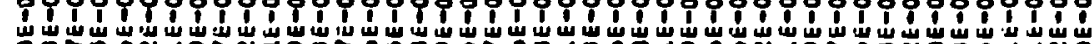
ON N N N

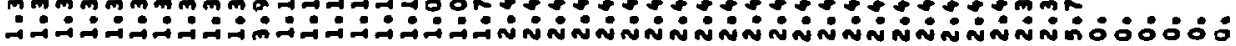

tे

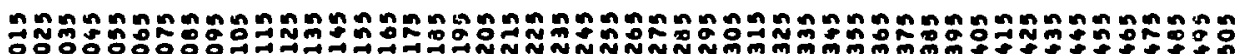
N

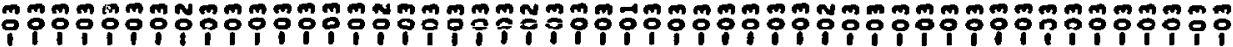

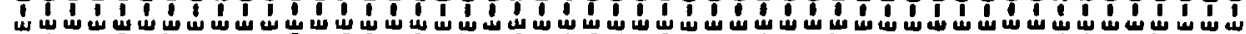

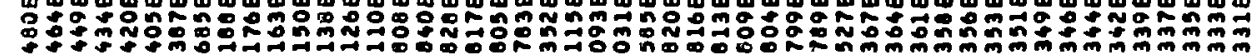

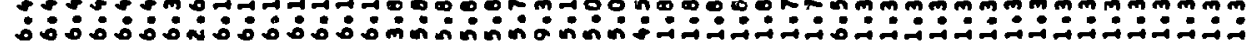

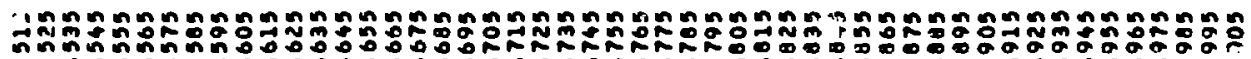
I

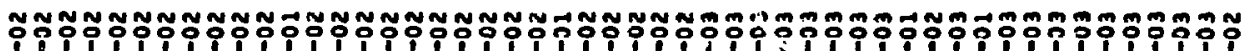

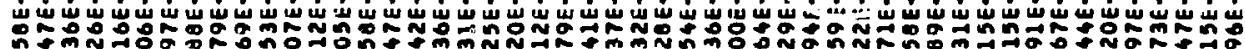

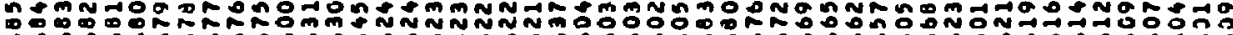

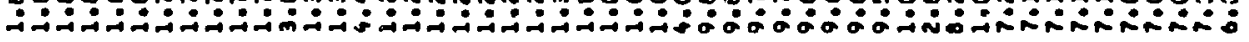

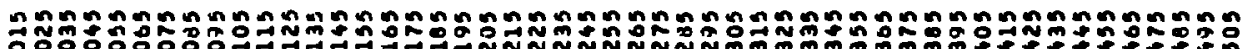

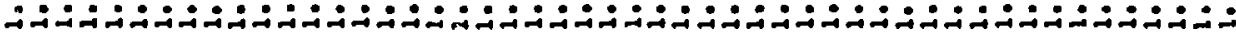

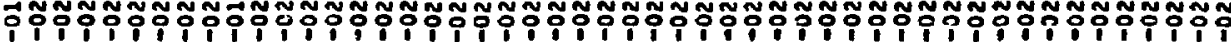

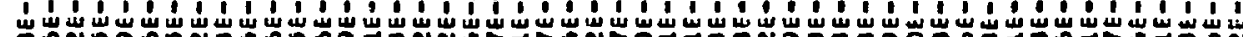

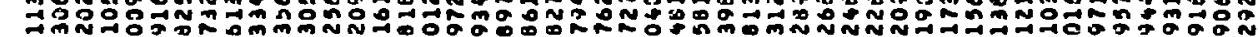

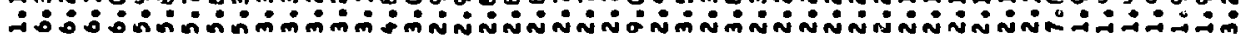

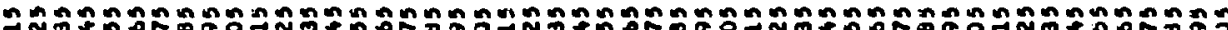

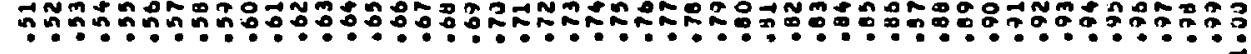

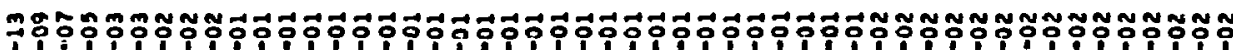

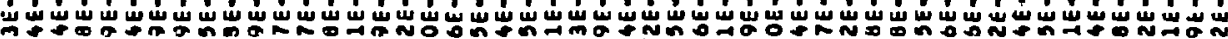

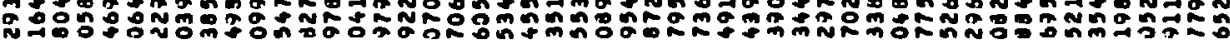
NÁ: 
$\stackrel{3}{3}$

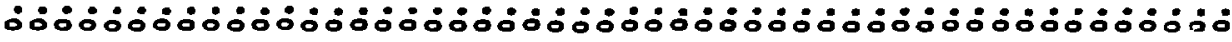

ל

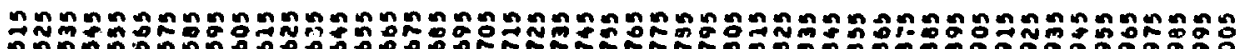

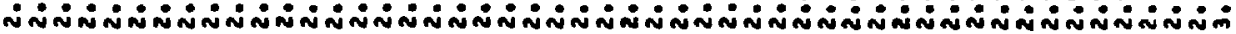

క

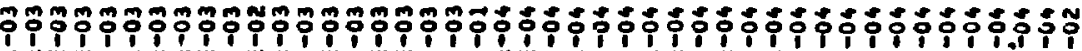

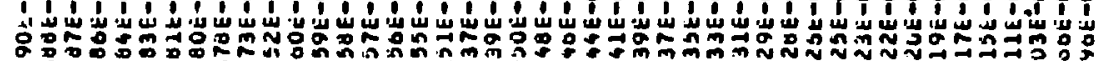

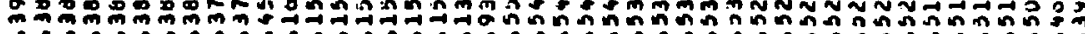

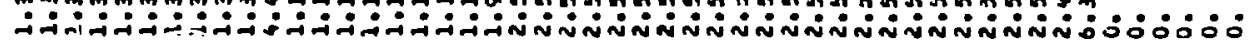

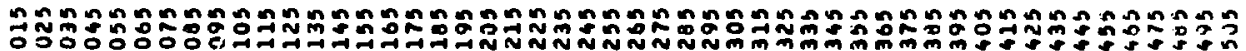

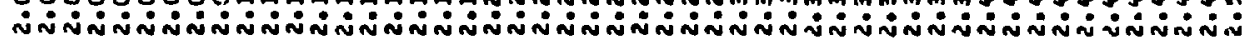

$\stackrel{\mathbf{4}}{\mathbf{4}}$

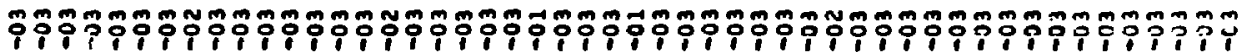

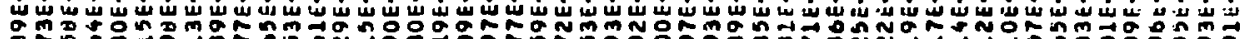

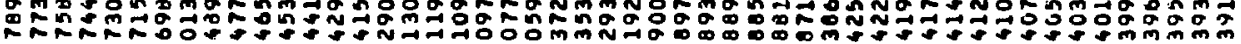

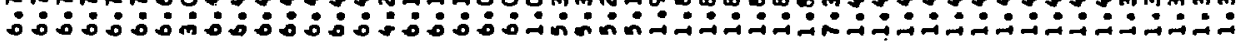

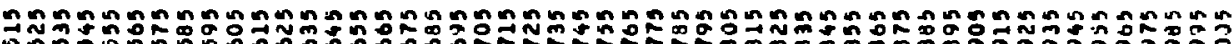

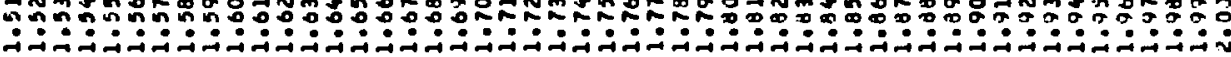

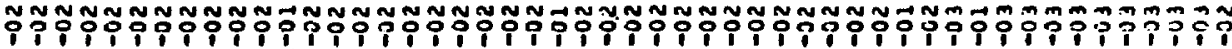

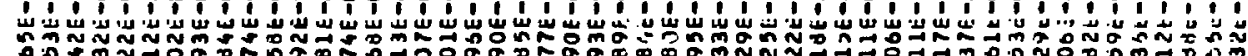

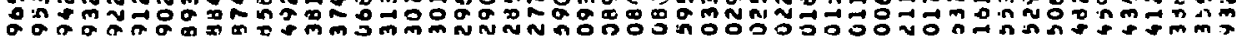

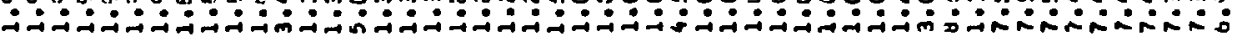

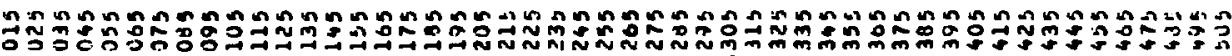

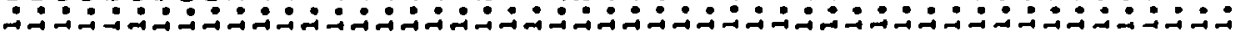

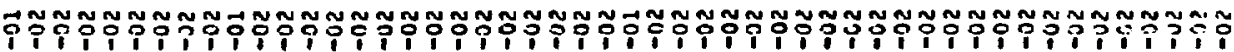

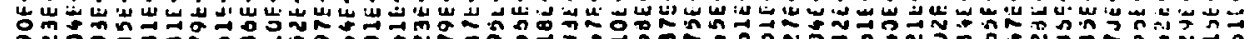

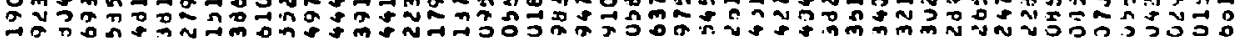

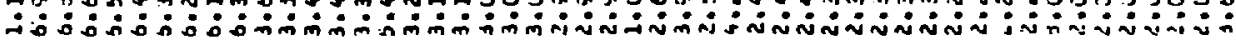

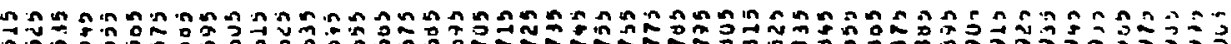

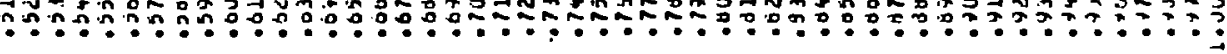

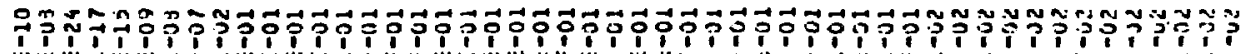

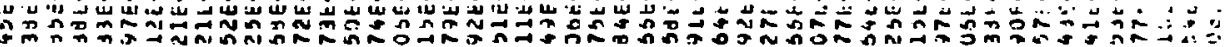
n? 
$\stackrel{3}{3}$

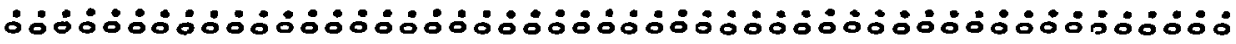

产

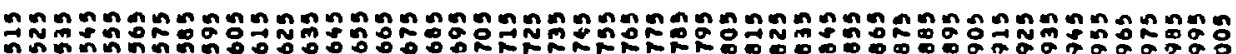

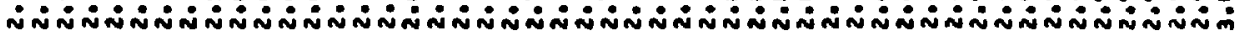

岁

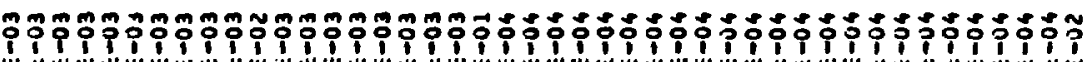

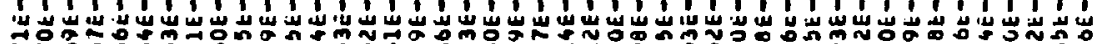

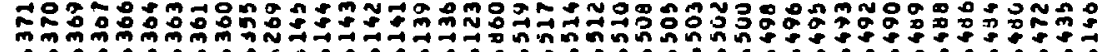

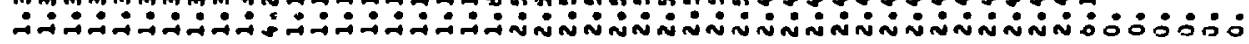

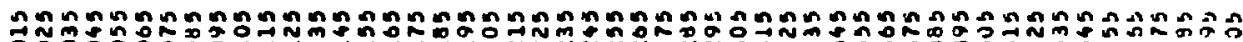

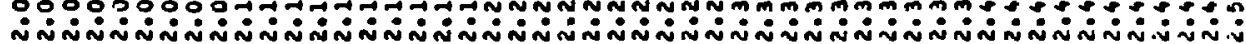

$\int^{x}$

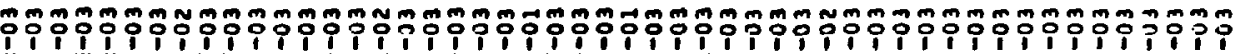

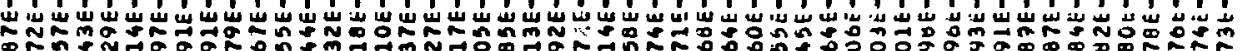

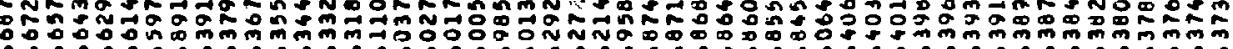
象:

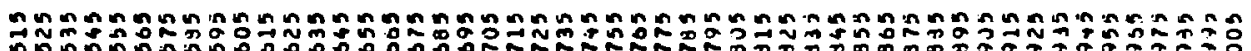

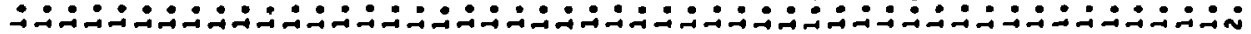

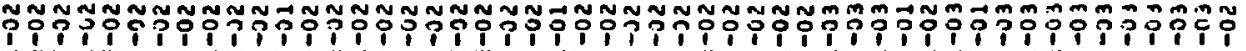

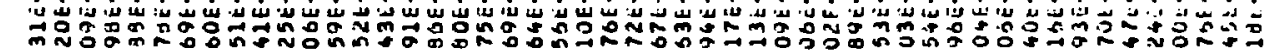

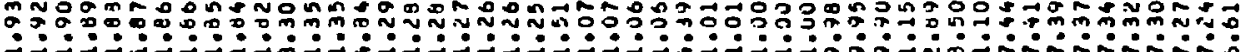

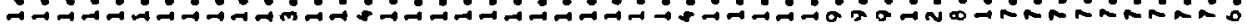

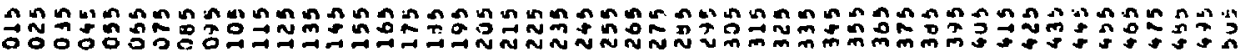

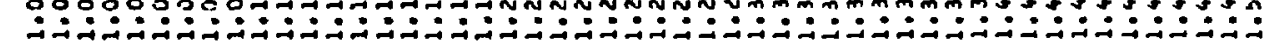

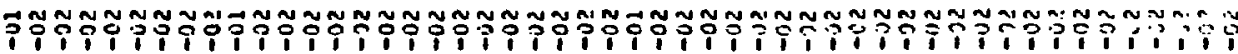

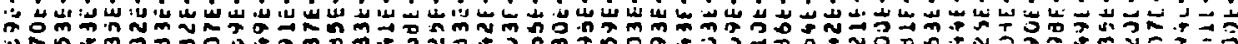

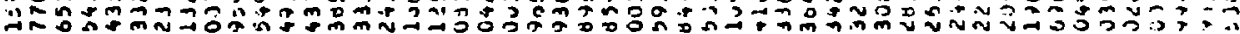

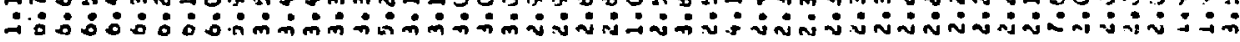

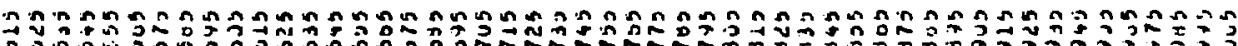

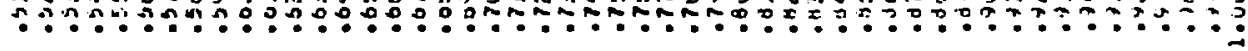

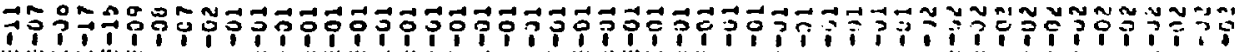

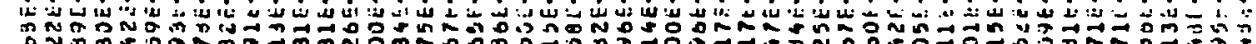

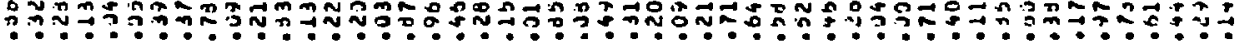

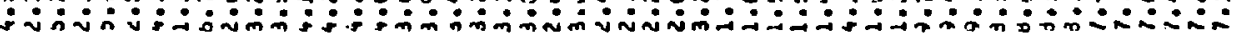

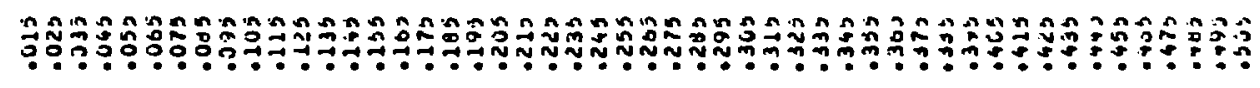




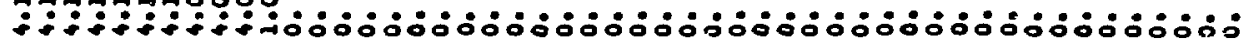

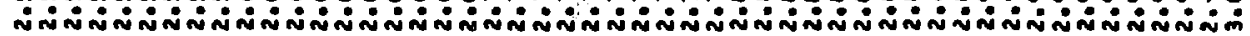

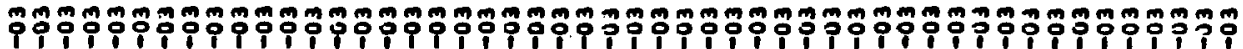

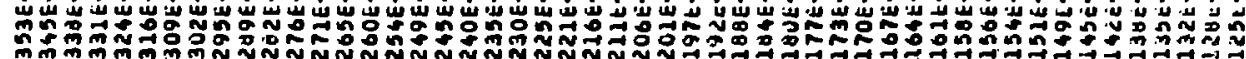

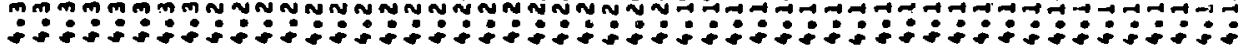

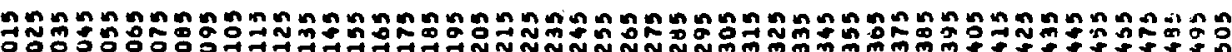

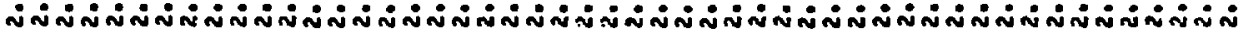

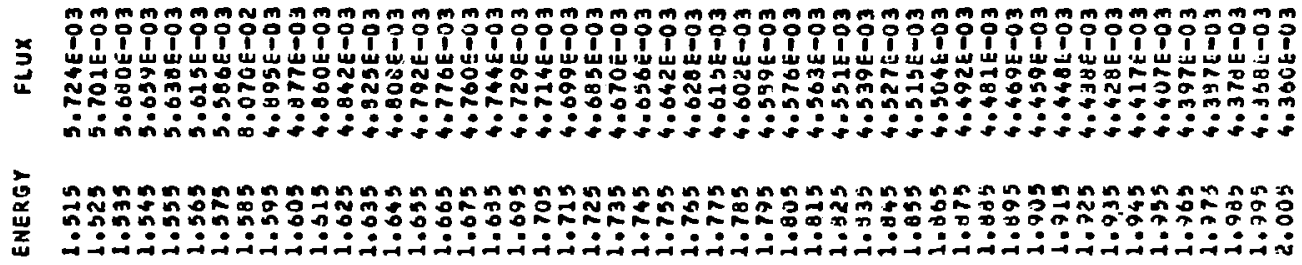

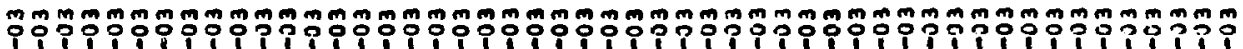

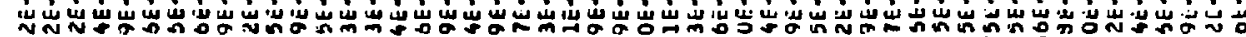

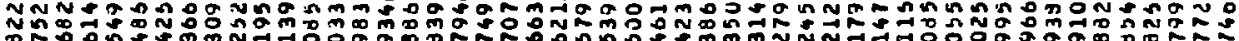

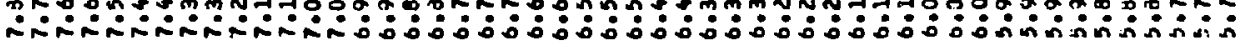

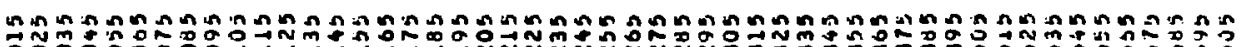

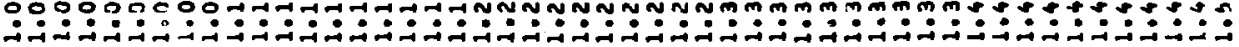

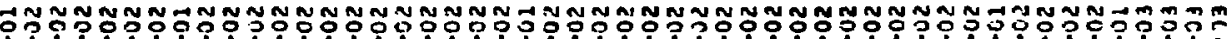
ipipipipipipipipipipipipipipipipipipipipipis

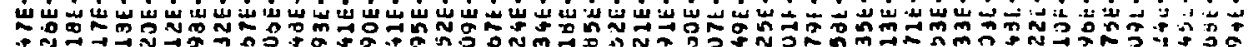

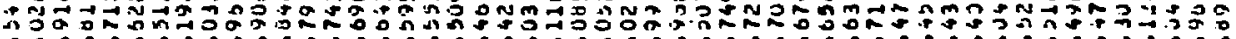

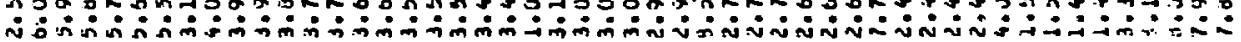

คิำ

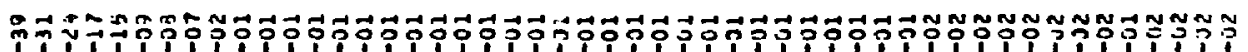

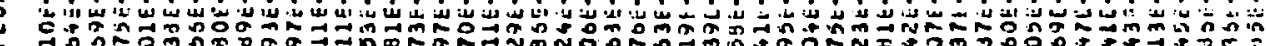

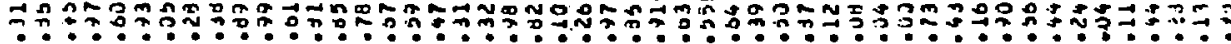

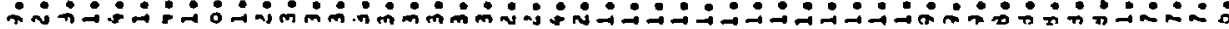

